UNIVERSIDADE DE BRASÍLIA

FACULDADE DE TECNOLOGIA

PÓS-GRADUACÃO EM CIÊNCIAS MECÂNICAS

DEPARTAMENTO DE ENGENHARIA MECÂNICA

\title{
IMPLEMENTAÇÃO DE FORMULAÇÕES DO MÉTODO DOS ELEMENTOS DE CONTORNO PARA ASSOCIAÇÃO DE PLACAS NO ESPAÇO
}

KERLLES RAFAEL PEREIRA SOUSA

ORIENTADOR: ÉDER LIMA DE ALBUQUERQUE CO-ORIENTADOR: DALMO INÁCIO GALDEZ COSTA

DOUTORADO EM CIÊNCIAS MECÂNICAS

PUBLICAÇÃO: ENM.TD - 00/0000

BRASÍLIA/DF: MARÇO-2016 
UNIVERSIDADE DE BRASÍLIA

FACULDADE DE TECNOLOGIA

PÓS-GRADUACÃO EM CIÊNCIAS MECÂNICAS

DEPARTAMENTO DE ENGENHARIA MECÂNICA

\section{IMPLEMENTAÇÃO DE FORMULAÇÕES DO MÉTODO DOS ELEMENTOS DE CONTORNO PARA ASSOCIAÇÃO DE PLACAS NO ESPAÇO}

\section{KERLLES RAFAEL PEREIRA SOUSA}

Relatório submetido como requisito parcial para obtenção do grau de Doutor em Ciências Mecânicas pelo Departamento de Engenharia Mecânica da Universidade de Brasília.

Banca Examinadora:

Prof. Éder Lima de Albuquerque, UnB/ENM

Prof. Simone dos Santos, UFPI/CT

Prof. Carla Tatiana Mota Anflor, UnB/FGA

Prof. Marcus Vinicius Girão de Morais, UnB/ENM

Prof. William Taylor Matias Silva, UnB/ENC

Brasília, 11 de março de 2016. 
FICHA CATALOGRÁFICA 


\section{Dedicatória}

À Deus, minha família, amigos, colegas de trabalho pelo apoio, força, incentivo, companheirismo e amizade. Sem eles nada disso seria possível. 


\section{Agradecimentos}

- Inicialmente, à Deus pela vida.

- À FAPEMA, pelo apoio financeiro durante o desenvolvimento deste trabalho.

- Ao meu orientador, Professor Éder Lima de Albuquerque, pela extraordinária orientação, comprometimento, apoio, amizade, paciência, por tantos conhecimentos transmitidos.

- A minha esposa, Josilene, aos meus pais, Reginaldo, João e Pêdra, e irmãos, Kerllesvaldo e Jefferson que, sempre incentivaram meus estudos, pelo apoio e participação em todas as minhas pequenas conquistas.

- Aos amigos André, Dalmo, Afonso, Bruno pela amizade, conhecimento e por estarem sempre dispostos a me ajudar no que fosse necessário, além das brincadeiras.

- A todos aqueles que, de forma direta ou indireta, contribuiram para a realização deste trabalho e que aqui não foram citados. 
A gratidão é, definitivamente, a forma de trazer mais para sua vida.

(Marci Shimoff) 


\section{Resumo}

Sousa, Kerlles Rafael Pereira, Implementação de Formulações do Método dos Elementos de Contorno para a Associação de Placas no Espaço. Brasília, 2016. Tese de Doutorado, Faculdade de Tecnologia, Universidade de Brasília.

Este trabalho apresenta uma formulação estática e dinâmica do método dos elementos de contorno para análise de estruturas formadas pela associação espacial de placas finas. As formulações dos elementos de contorno para elasticidade plana e flexão de placas finas de materiais isotrópicos são associadas, obtendo-se uma estrutura plana denominada de sub-região. O contorno desta região é dividido em pequenos pedaços, os elementos de contorno. Cada um deste elemento possui um ponto de colocação (elementos de contorno constante), onde as equações integrais de contorno são aplicadas. Uma sub-região contém simultaneamente os estados de flexão em placas finas e de elasticidade plana (chapa) e possui quatro graus de liberdade por nó, sendo eles: deslocamento normal, tangencial e transversal e rotação normal. O modelo final assume uma associação dessas sub-regiões no espaço. Cada sub-região é tratada via MEC. As equações de cada sub-região, após as transformações de coordenadas, são acopladas através da compatibilidade de deslocamentos e rotações e equilíbrio de forças e momentos. A fim de calcular os elementos das matrizes de influência, as integrais de contorno ao longo dos elementos serão obtidos numericamente. Na formulação dinâmica, os termos de inércia são considerados como forças de corpo, o que gera integrais de domínio na formulação. Estas integrais de domínio foram transformadas em integrais de contorno usando o método da integração radial. Dessa forma, a principal contribuição deste trabalho é a associação da formulação do método dos elementos de contorno de placas finas e elasticidade plana para problemas estáticos e dinâmicos onde, somente o contorno será discretizado em elementos de contorno constante. Com objetivo de validar a formulação desenvolvida, vários exemplos numéricos são analisados e, os resultados obtidos são comparados com Ansys e soluções disponíveis na literatura. Apesar da formulação ter usado elementos constantes, na maioria dos casos os resultados obtidos mostraram boa concordância com os resultados da literatura.

Palavras chaves: Método dos elementos de contorno, placas fina, material isotrópico, sub-regiões. 


\section{Abstract}

Sousa, Kerlles Rafael Pereira, Implementation of Boundary Element Method Formulation for Plate Association in Space. Brazilia, 2016. PhD Thesis, Faculty of Technology, University of Brazilia.

This work presents a dynamic and static formulation of the boundary element method for analysis of spatial structures formed by the association of thin plates. The formulations of the boundary element for plane elasticity and bending of thin plates of isotropic materials are associated with, obtaining a flat structure called the sub-region. A sub-region contains both the states of flexion and extension in thin plates and has four degrees of freedom per node, namely: normal, tangential and transverse displacements and normal rotation. The final model assumes a combination of these sub-regions in space. The equations of each sub-region, after the coordinate transformations, are coupled by displacement and rotation compatibility and forces and momentum equilibrium. In order to calculate the coefficients of the matrix of influence the integrals over elements are obtained numerically. In the dynamic formulation, inertia terms are considered as body forces, generating domain integrals in the formulation. These integrals are transformed into boundary integrals by the radial integration method. Thus, the main contribution of this work is the association of the method formulation of thin plate boundary element and plane elasticity for static and dynamic problems where only the boundary will be discretized in constant boundary element. In order to validate the proposed formulation, various numerical examples are analyzed, and the results obtained are compared with Ansys and solutions available in the literature. Although the use of constant elements, there is a good agreement with literature in the majority of numerical examples.

Key words: Boundary element method, thin plates, isotropic material, subregions. 


\section{Sumário}

1 Introdução 1

1.1 A evolução da análise de estruturas . . . . . . . . . . . . . . . . 1

1.2 O método dos elementos de contorno . . . . . . . . . . . . . . 2

1.3 Considerações sobre placas . . . . . . . . . . . . . . . . 3

1.4 Revisão bibliográfica . . . . . . . . . . . . . . . . . . . . . 4

1.5 Organização dos capítulos . . . . . . . . . . . . . . . 7

2 Teoria da Elasticidade Linear $\quad 9$

2.1 Introdução . . . . . . . . . . . . . . . . . . . . 9

2.2 Tensão: Tensor de tensão . . . . . . . . . . . . . . . . . . . . 9

2.3 Relações Deformação - Deslocamento . . . . . . . . . . . . . . . . . 11

2.4 Equações constitutivas . . . . . . . . . . . . . . . . . . . . . . . . . . 13

2.5 Equações de equilíbrio . . . . . . . . . . . . . . . . . . . . . . . . . 13

2.6 Equações compatibilidade . . . . . . . . . . . . . . . . . . . . . 14

3 Método dos Elementos de Contorno para elasticidade plana $\quad 16$

3.1 Formulação integral de contorno para elasticidade . . . . . . . . . . 16

3.2 Soluções fundamentais . . . . . . . . . . . . . . . . . . . . . . . . . 19

3.3 Equações integrais singulares . . . . . . . . . . . . . . . . . . . . . . . . 19

3.4 Elementos de contorno constante . . . . . . . . . . . . . . . . . . . 21

3.5 Formulação dos Elementos de Contorno Discretizada . . . . . . . . . . 23

4 Teoria de Placas $\quad 25$

4.1 Considerações gerais . . . . . . . . . . . . . . . . . . . . 25

4.2 Hipóteses básicas . . . . . . . . . . . . . . . . . . . . . . . . . . 27

4.3 Placas de Kirchhoff . . . . . . . . . . . . . . . . . . . . . . . . . . 28

4.4 Placas isotrópicas . . . . . . . . . . . . . . . . . . . 29

4.4.1 Relações deslocamento-deformação e curvatura . . . . . . . . . . 30

4.4.2 Tensões e Resultantes de Tensões . . . . . . . . . . . . . . . . . 31

4.5 Equações constitutivas . . . . . . . . . . . . . . . . . . 33 
4.5.1 Equação diferencial . . . . . . . . . . . . . . . . 35

4.6 Cálculo da rigidez à flexão em direções arbitrárias . . . . . . . . . . . 36

4.7 Condições de contorno . . . . . . . . . . . . . . . . . . . 38

4.8 Soluções fundamentais isotrópicas . . . . . . . . . . . . . . . . . . . . . 39

5 Equações do Método dos Elementos de Contorno para Placas de Kir$\begin{array}{ll}\text { chhoff } & 41\end{array}$

5.1 Formulação integral . . . . . . . . . . . . . . . . . . . . . 41

5.2 Equação Matricial . . . . . . . . . . . . . . . . . . . 48

6 Transformação das Integrais de Domínio em Integrais de Contorno 50

6.1 Introdução . . . . . . . . . . . . . . . . . . . 50

6.2 Transformação Exata . . . . . . . . . . . . . . . . . . 51

6.3 Método da integração radial - RIM . . . . . . . . . . . . . . 53

6.4 Método de Houbolt . . . . . . . . . . . . . . . . . . . . . . . 56

7 Associação de Macro-elementos Via Sub-regiões do MEC 58

7.1 Introdução . . . . . . . . . . . . . . . . . . . . . . . 58

7.2 Formulação do macro-elemento. . . . . . . . . . . . . . . . . . 59

7.2.1 Equações Algébricas do Macro-Elemento . . . . . . . . . . . . 59

7.2.2 Montagem do sistema de equações . . . . . . . . . . . . . 61

7.3 Associação espacial de macro-elementos . . . . . . . . . . . . . . 61

7.3.1 Sistema de coordenadas . . . . . . . . . . . . . . 61

7.3.2 Compatibilização de momentos e rotações . . . . . . . . . . . . 64

7.3.3 Associação de macro-elementos via método de sub-regiões do MEC 64

7.4 Aspectos computacionais . . . . . . . . . . . . . . . . . . 67

8 Resultados Numéricos para Problemas Estáticos $\quad 68$

8.1 Introdução . . . . . . . . . . . . . . . . . . . . . . . 68

8.2 Elasticidade Plana . . . . . . . . . . . . . . . . . . . . . 69

8.2.1 Chapa tracionada . . . . . . . . . . . . . . . 69

8.2 .2 Placa apoiada . . . . . . . . . . . . . . . . . . 71

8.2.3 Placa em balanço . . . . . . . . . . . . . . . 74

8.2.4 Placa engastada . . . . . . . . . . . . . . . 76

8.3 Associação em $L$. . . . . . . . . . . . . . . . . . . . . . . . . . . . . . . . . . . . 79

8.3.1 Associação em $L$ em ângulos não-retos . . . . . . . . . . . . . . 82

8.3.2 Viga em formato de caixa . . . . . . . . . . . . . . 87

8.4 Associação em $L$ com carregamento lateral . . . . . . . . . . . . . . . . 93 
9 Resultados Numéricos de Problemas Transientes 96

9.1 Introdução . . . . . . . . . . . . . . . . . . . 96

9.2 Resultados numéricos . . . . . . . . . . . . . . . 96

9.2.1 Placa apoiada . . . . . . . . . . . . . . . . . 96

9.2 .2 Placa engastada . . . . . . . . . . . . . . . 98

9.2 .3 Placa em balanço . . . . . . . . . . . . . . . . . 100

9.2.4 Tira infinita sob tração uniforme . . . . . . . . . . . . 102

9.2.5 Associação em $L$. . . . . . . . . . . . . . . . . . . 104

10 Considerações finais 106

10.1 Conclusões . . . . . . . . . . . . . . . . . . . . 106

10.2 Sugestões para trabalhos futuros . . . . . . . . . . . . . . . 108 


\section{Símbolos}

\section{Letras gregas}

$\alpha=$ Ângulo.

$\varepsilon=$ Deformação normal.

$\phi=$ Ângulo.

$\Gamma=$ Contorno.

$\gamma=$ Deformação cisalhante.

$\mu=$ Raíz do polinômio característico.

$\nu=$ Razão de Poisson.

$\Omega=$ Domínio.

$\theta=$ Ângulo.

$\rho=$ Distância.

$\sigma=$ Tensão normal.

$\tau=$ Tensão cisalhante.

\section{Letras arábicas}

$b=$ Força de corpo.

$C, K=$ Constantes.

$\mathbf{D}=$ Matriz de rigidez de flexão.

$\mathbf{D}^{\prime}=$ Matriz $\mathbf{D}$ transformada.

$d_{i}=$ Parte real de $\mu$.

$E=$ Módulo de elasticidade.

$e_{i}=$ Parte imaginária de $\mu$.

$g=$ Força elementar.

$M, m=$ Momentos.

$N=$ Função de interpolação.

$\mathbf{n}=$ Vetor normal ao contorno.

$Q=$ Ponto campo.

$\mathbf{Q}=$ Matriz de rigidez.

$\overline{\mathbf{Q}}=$ Matriz de rigidez transformada. 
$q=$ Força distribuída.

$R_{i}=$ Função.

$r_{i}, s_{i}, q_{i}, p_{i}=$ Constantes.

$S_{i}=$ Função.

$\mathbf{T}=$ Matriz de transformação.

$t=$ Espessura da placa.

$u, v=$ Deslocamentos no plano.

$V=$ Força cortante equivalente de Kirchhoff.

$w=$ Deslocamento transversal.

$z=$ Distância transversal do plano médio à um ponto.

\section{Subscritos}

$\Gamma=$ Contorno

$\Omega=$ Domínio.

1, 2, $3=$ Direções principais.

$c=$ Compressão, elemento contínuo.

$d=$ Elemento descontínuo.

$L=$ Direção longitudinal às fibras.

$n=$ Direção normal.

$s=$ Direção tangencial.

$T=$ Direção transversal às fibras.

$t=$ Tração.

$x, y, z=$ Eixos do sistema de coordenadas.

\section{Sobrescritos}

1, 2, 3 = Nós do elemento.

$*=$ Soluções fundamentais. 


\section{Lista de Figuras}

2.1 Estado geral de tensão. . . . . . . . . . . . . . . . . . . . . 10

2.2 Deformações: campo de deslocamentos. . . . . . . . . . . . . . . . 11

2.3 Configuração deforma de um corpo . . . . . . . . . . . . . . 12

3.1 Ponto de carregamento (Q) e de deslocamento (P). . . . . . . . . . 19

3.2 Ponto fonte localizado no contorno, circundado por uma região semi-

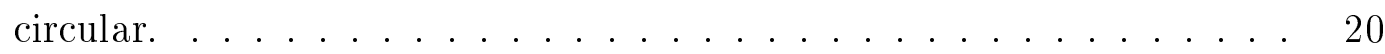

3.3 Ilustração de cinco tipos de elementos: a) constante b)lineares contínuos, c) lineares descontínuos, d) quadráticos contínuos, e) quadráticos descontínuos. . . . . . . . . . . . . . . . . . . 22

4.1 Placa fina. . . . . . . . . . . . . . . . . . 26

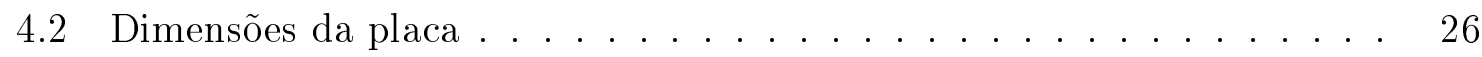

4.3 Flecha . . . . . . . . . . . . . . . . . . 27

4.4 Comportamento Geral de Placas. . . . . . . . . . . . . . . . . . . 29

4.5 Resultantes de tensões. . . . . . . . . . . . . . . . . . . . . . . 32

4.6 Equilíbrio de forças e momentos em uma placa . . . . . . . . . . . . 34

4.7 Elemento de placa submetido a momentos nas bordas. . . . . . . . . . . 37

4.8 Elemento de placa submetido a momentos em direções arbitrárias. . . . 37

4.9 Momento volvente no contorno. Paiva (1987) . . . . . . . . . . 38

5.1 Placa finita contida em uma placa infinita. . . . . . . . . . . . . 41

5.2 Canto $i$ do contorno da placa. . . . . . . . . . . . . . . 45

6.1 Transformação da integral de domínio em integral de contorno. . . . . . 52

6.2 Posição dos pontos no domínio. . . . . . . . . . . . . . . . . . 55

7.1 Deslocamentos e esforços em um nó genérico $\Psi$ de um macro-elemento. $\quad 59$

7.2 Sistema de Coordenadas. . . . . . . . . . . . . . . . . . . . . . 62

7.3 Compatibilização de momentos e rotações nos macro-elementos (neste caso $\beta=1) \ldots \ldots \ldots \ldots \ldots \ldots \ldots \ldots \ldots$

7.4 Associação de dois macro-elementos. . . . . . . . . . . . . . 65 
8.1 Ilustração da placa submetida a força axial. . . . . . . . . . . . 69

8.2 Ilustração da placa apoiada. . . . . . . . . . . . . . . . . . . . 71

8.3 (a) Deslocamento na direção $z$ para a placa apoiada usando 1 sub-região.

(b) Deslocamento na direção $z$ para a placa apoiada usando 2 sub-regiões. 72

8.4 Ilustração da placa em balanço. . . . . . . . . . . . . . . . . . . . . . . 74

8.5 (a) Deslocamento na direção $z$ para a placa em balanço usando 1 subregião. (b) Deslocamento na direção z para a placa em balanço usando 2 sub-regiões. . . . . . . . . . . . . . . . . . 75

8.6 Ilustração da placa engastada. . . . . . . . . . . . . . . . . . 76

8.7 (a) Deslocamento na direção z para a placa engastada usando 1 subregião. (b) Deslocamento na direção $z$ para a placa engastada usando 2 sub-regiões. . . . . . . . . . . . . . . . . 77

8.8 Resultados obtidos usando o MEF através do ANSYS. . . . . . . . . . 78

8.9 Dimensões e condições de contorno para a estrutura em $L$ (Dirgantara and Aliabadi, 2001) . . . . . . . . . . . . . .

8.10 (a) Distribuição do deslocamento total para a estrutura em $L$ obtidos com a formulação do MEC de placa fina. (b) Distribuição do deslocamento total para a estrutura em $L$ obtidos com a formulação do MEC para placa espessa. . . . . . . . . . . . . . . . . .

8.11 As dimensões e condições de contorno para a estrutura unida por um ângulo arbitrário $\theta$ (Dirgantara and Aliabadi, 2001) . . . . . . . . . .

8.12 (a) Deslocamento total para a estrutura unida por um ângulo $\theta=$ $91^{\circ}$ obtido com o MEC para placa fina com 160 elementos de contorno.

(b) Deslocamento total para a estrutura unida por um ângulo $\theta=$ $91^{\circ}$ obtido com o MEC para placa espessa. . . . . . . . . . . . . . .

8.13 (a) Deslocamento total para a estrutura unida por um ângulo $\theta=$ $95^{\circ}$ obtido com a formulação do MEC para placa fina com 160 elementos de contorno. (b) Deslocamento total para a estrutura unida por um ângulo $\theta=95^{\circ}$ obtido com o MEC para placa espessa. . . . . . . . . . .

8.14 (a) Deslocamento total para a estrutura unida por um ângulo $\theta=$ $120^{\circ}$ obtido com a formulação do MEC para placa fina com 160 elementos de contorno. (b)Deslocamento total para a estrutura unida por um ângulo $\theta=120^{\circ}$ obtido com o MEC para placa espessa. . . . . . . . .

8.15 Dimensões e condições de contorno para a viga em forma de caixa em balanço. . . . . . . . . . . . . . . . .

8.16 Deslocamento total para a viga em formato de caixa em balanço para $L_{3}=0,05 \mathrm{~m}$ obtido com MEC para placas finas. . . . . . . . . . . 
8.17 Deslocamento na direção $z$ para a viga em formato de caixa em balanço para $L_{3}=0,05 \mathrm{~m}$ com 96 elementos.

8.18 Deslocamento na direção $z$ para a viga em formato de caixa em balanço para $L_{3}=0,1 \mathrm{~m}$ com 192 elementos. . . . . . . . . . . . . . . 90

8.19 Deslocamento na direção $z$ para a viga em formato de caixa em balanço para $L_{3}=0,2 \mathrm{~m}$ com 96 elementos. . . . . . . . . . . . . . .

8.20 Deslocamentos $u_{2}$ para o lado alinhado com a direção $x_{2}$ localizados nas coordenadas $x_{1}=x_{3}=0 \mathrm{~m}$ para $L_{3}=0,05 \mathrm{~m}$. . . . . . . . . . . . . 91

8.21 Deslocamentos em $x_{2}=2 \mathrm{~m}$ para a viga em formato de caixa em balanço sujeita a um momento para $L_{3}=0,1 \mathrm{~m}$. . . . . . . . . . . . . . . . . 91

8.22 Deslocamentos em $x_{2}=2 \mathrm{~m}$ para a viga em formato de caixa em balanço sujeita a um momento para $L_{3}=0,2 \mathrm{~m} \ldots \ldots$. . . . . . . . . . . . 92

8.23 Estrutura L-shaped. . . . . . . . . . . . . . . . . . . . . . . . 93

8.24 Resultados obtidos com MEC: a) placa fina e b) placa espessa. . . . . . 94

8.25 Resultados para a placa superior projetados no plano $x_{1}-x_{2}$. . . . . . 95

9.1 Ilustração da placa apoiada. . . . . . . . . . . . . . . . . . . . 97

9.2 Variação no tempo do deslocamento no ponto central da placa quadrada simplesmente apoiada submetido a uma carga repentinamente aplicada. 97

9.3 Ilustração da placa engastada. . . . . . . . . . . . . . . . . . . . . . . . 98

9.4 Variação no tempo do deslocamento no ponto central da placa quadrada engastada submetido a uma carga repentinamente aplicada. . . . . . . 99

9.5 Ilustração da placa em balanço. . . . . . . . . . . . . . . . . . . . . . 100

9.6 Variação no tempo do deslocamento transversal no ponto $A$ da placa quadrada em balanço submetido a uma carga repentinamente aplicada. 101

9.7 Ilustração da tira infinita sob tração uniforme. . . . . . . . . . . . . . . 102

9.8 Ilustração da seção da tira infinita sob tração uniforme. . . . . . . . . . . 102

9.9 Carregamento tipo função degrau. . . . . . . . . . . . . . . . . . . . . . 103

9.10 Variação do deslocamento no tempo da tira infinita sob carga com passo de tempo uniforme. . . . . . . . . . . . . . . . . . . 103

9.11 Dimensões e condições de contorno para a estrutura em $L$ Dirgantara and Aliabadi (2001). . . . . . . . . . . . . . . . . 104

9.12 Variação do deslocamento no tempo da tira infinita sob carga com passo de tempo uniforme. . . . . . . . . . . . . . . . 105 


\section{Lista de Tabelas}

4.1 Condições de contorno. . . . . . . . . . . . . . . . . . . . . 38

8.1 Resultados obtidos para placa - força axial. . . . . . . . . . . . 70

8.2 Resultados obtidos para placa apoiada. . . . . . . . . . . . . 73

8.3 Resultados obtidos para placa em balanço. . . . . . . . . . . . . . . . . 74

8.4 Resultados obtidos para placa em balanço. . . . . . . . . . . . . . 78

8.5 Soluções analíticas e numéricas do deslocamento na direção $z$ calculado na ponta da placa. . . . . . . . . . . . . . . . . . . . 79

8.6 Resultados obtidos para os deslocamentos da estrutura em $L$. . . . . . 80

8.7 Soluções analíticas do deslocamento para três diferentes ângulos calculados para um ponto na extremidade carregada da placa superior. . . . 82

8.8 Resultados obtidos para os deslocamentos da estrutura em $L$ unida por um ângulo $\theta=91^{\circ} \ldots \ldots \ldots$. . . . . . . . . . . . . . . . 83

8.9 Resultados obtidos para os deslocamentos da estrutura em $L$ unida por um ângulo $\theta=95^{\circ}$. . . . . . . . . . . . . . . . . . . . 83

8.10 Resultados obtidos para os deslocamentos da estrutura em $L$ unida por um ângulo $\theta=120^{\circ} \ldots \ldots \ldots \ldots$. . . . . . . . . . . 87 


\section{Capítulo 1}

\section{Introdução}

\subsection{A evolução da análise de estruturas}

As civilizações, desde tempos antigos, transforma recursos disponíveis a sua volta de modo a facilitar suas necessidades. Desta forma, o ser humano tem produzido moradias, ferramentas auxiliares, meios de locomoção, computadores e máquinas modernas. Com a evolução ao longo dos anos dos processos de concepção das manufaturas, os conhecimentos empírico/científico adquiridos pelo homem na transformação dos recursos deu origem à hoje conhecida engenharia. Em linhas gerais, a engenharia aplica esses conhecimentos na produção de manufaturas.

Alguns motivos influenciaram o ser humano a questionar e investigar o comportamento dos materiais. Entre esses, podem ser citados: falhas estruturais que ocasionaram perdas humanas e materiais, a otimização de formas estruturais visando a economia dos recursos e a adequabilidade do comportamento de diferentes materiais a diferentes utilizações da humanidade. Nesse contexto, surge a ciência/engenharia de estruturas.

A existência destes projetos vem da aplicação de técnicas para escolha de materiais e o dimensionamento de estruturas. A formalização destes projetos e, consequentemente, da engenharia estrutural, é obtida através de teorias científicas que permitem aos engenheiros estabelecerem as forças e solicitações que podem atuar com segurança nas estruturas ou em seus componentes, e definir os materiais adequados e as dimensões necessárias da estrutura e seu componentes sem que estes sofram efeitos prejudiciais ao seu bom funcionamento.

O início da formalização teórica que relaciona a aplicação de forças às deformações foi feito por volta de 1678 por Robert Hooke, cujo sobrenome é extremamente familiar à maioria dos estudantes das engenharias mecânica e civil. Apesar desse longínquo início, grandes avanços na teoria da elasticidade não foram obtidos até o ano de 1821 quando 
Navier publicou seus estudos acerca das equações de equilíbrio. Este desenvolvimento foi seguido por Cauchy que estudou as equações básicas da elasticidade e desenvolveu o conceito de tensão em um ponto. Ao longo desses séculos, vários matemáticos e cientistas ilustres deram suas contribuições para formalizar a engenharia estrutural tal como se entende hoje. Até o início do século XX pode-se citar, dentre outros, Bernoulli, Lord Kelvin, Poisson, Lamé, Green, Saint-Venant, Betti, Airy, Kirchhoff, Rayleigh, Love, Timoshenko. A totalidade destes autores desenvolveu a forte base matemática presente nos livros e formulações usadas atualmente, sendo muito dos seus desenvolvimentos homenageados em denominações como a viga de Timoshenko, teoria de placas de Kirchhoff-Love, princípio de Saint-Venant, coeficiente de Poisson, solução fundamental de Kelvin para elasticidade Sadd (2009).

A engenharia tem como um de seus principais objetivos a prevenção de falhas em estruturas que possam a ocasionar perdas humanas e materiais. Assim, por meio da avaliação do comportamento mecânico das estruturas e de sua interação com o meio externo, modelos matemáticos foram desenvolvidos baseados em leis físicas do comportamento de sólidos e fluidos.

\subsection{O método dos elementos de contorno}

Desde a década de 1960, uma poderosa ferramenta despontou no mundo da engenharia: o computador, embora inicialmente apenas em institutos de pesquisa e universidade. A potencialidade de realizar repetidos cálculos em um tempo pequeno e de sistematizar certas atividades, levou os pesquisadores a aplicar alguns conceitos como análise matricial de estruturas a uma configuração mais abrangente. Surgiram assim, os métodos numéricos como: o Método dos Elementos Finitos (MEF), o Método das Diferenças Finitas (MDF) e o Método das Equações Integrais. Mas, este último, para distinguí-lo dos outros métodos que envolviam equações integrais, ele foi finalmente chamado de Método dos Elementos de Contorno.

De acordo com Brebbia and Dominguez (1989), o Método dos Elementos de Contorno (MEC) tem sido estabelecido como um método numérico alternativo ao Método dos Elementos Finitos (MEF), em casos particulares onde um melhor rigor é requerido devido a problemas como concentração de tensões ou onde o domínio se estende ao infinito. Isto se deve à sua simplicidade e redução na dimensionalidade do problema. Por exemplo, um problema bidimensional reduz-se somente a uma curva correspondente ao contorno do domínio que precisa ser discretizada em elementos e um problema tridimensional reduz-se a uma superfície do domínio que necessita ser discretizada. Isto significa que, comparado à análise de um método de domínio tipo MEF, uma análise 
do método dos elementos de contorno resulta em uma substancial redução na preparação dos dados e em um sistema algébrico de equações muito menor a ser resolvido numericamente.

O MEC pode ser aplicado em uma ampla variedade de problemas de engenharia, tais como: mecânica da fratura, mecânica do contato, barreira acústica, proteção catódica (em casco de navios e torres de distribuição elétrica), e problemas de elasticidade.

\subsection{Considerações sobre placas}

As formulações de elementos de contorno têm sido aplicadas a problemas de flexão em placas isotrópicas, considerando a teoria de Kirchhoff, bem como teorias de placas deformáveis ao cisalhamento. Shi and Bezine (1990) apresentam uma análise por elementos de contorno de problemas de flexão de placa usando a solução fundamental proposta por Wu and Altiero (1981), baseadas nos pressupostos de flexão da placa de Kirchhoff.

Rajamohan and Raamachandran (1999) propuseram uma formulação na qual as singularidades são evitadas por pontos fontes colocados fora do domínio. Paiva et al. (2003) apresentaram um tratamento analítico para integrais singulares e hipersingulares da formulação proposta por Shi and Bezine (1990).

Placas deformáveis por cisalhamento tem sido analisadas usando o método dos elementos de contorno por Rashed (2000), Weën (1982) e Wang and Schweizerhof (1997). com a solução fundamental proposta por Wang and Schweizerhof (1995).

No método dos elementos de contorno para flexão de placas, a presença de forças distribuídas no domínio fazem aparecer na formulação as integrais de domínio. Com o objetivo de resolver estas integrais, o esquema de integração por células pode dar resultados precisos, como demonstrado por Shi and Bezine (1990) para problemas de flexão em placas anisotrópicas. Contudo, a discretização do domínio em células reduz uma das principais vantagens do método dos elementos de contorno que é a discretização somente do contorno. Uma alternativa para este procedimento foi apresentada por Rajamohan and Raamachandran (1999) que propuseram o uso de soluções particulares para aproximar a discretização do domínio. Entretanto, o uso de soluções particulares requer a busca de uma função que satisfaça a equação governante. Dependendo da complexidade da equação governante, esta função pode ser difícil de ser encontrada.

As integrais de domínio devido a cargas distribuídas podem ser transformadas em integrais de contorno pelo método da integração radial. Este método foi inicialmente apresentado por Venturini (1988) para problemas de flexão em placas isotrópicas. Recentemente, Gao (2002) estendeu o método para problemas de elasticidade isotrópica 
tridimensional e Albuquerque et al. (2006) para placa de Kirchhoff anisotrópica.

A adoção de hipóteses simplificadoras, visando analisar a placa como um elemento bidimensional, fez surgir diferentes teorias para verificar o comportamento geral desta superfície estrutural. Kirchhoff (1850) estabeleceu as hipóteses fundamentais da teoria de placas finas, derivando a expressão da energia potencial para uma placa inclinada e aplicando o princípio dos trabalhos virtuais para obter uma equação diferencial, onde a rigidez à flexão foi definida em termos do módulo de Young e coeficiente de Poisson. Adicionalmente, ele percebeu que as três condições de contorno naturais propostas por Poisson (1829) não eram compatíveis com a natureza de quarta ordem da equação diferencial obtida e mostrou que estas poderiam ser reduzidas a duas condições de contorno naturais. Esta teoria não leva em conta o efeito da deformação pelo esforço cortante, assumindo-se que, retas normais ao plano médio da placa permanecem normais após a deformação. As hipóteses apresentadas por Kirchhoff resultaram em uma equação diferencial de quarta ordem, na qual o deslocamento é dado em função de duas coordenadas no plano médio da placa. Esta equação pode ser uma eficiente representação do comportamento de placas finas para pequenos deslocamentos, apresentando boa precisão de resultados para uma grande variedade de carregamentos e geometrias. Entretanto, a teoria desenvolvida por Kirchhoff não apresenta bons resultados quando são analisadas placas de maior espessura. Mindlin (1951) formulou uma teoria para analisar placas moderadamente espessas onde, assumindo-se que as distorções que ocorrem na espessura são constantes, as tensões são obtidas a partir da geometria imposta para as deformações. O sistema de equações diferenciais obtido é de sexta ordem e também satisfaz as três condições de contorno requeridas. As formulações apresentadas por Kirchhoff e Mindlin podem ser consideradas como expressivas contribuições para o aprimoramento da teoria bidimensional de placas.

\subsection{Revisão bibliográfica}

Nesta tese, o tema - associação de placas finas através do MEC - será abordado e, a análise estática e dinâmica será o seu foco. Para tanto, os seguinte temas serão revisados:

- As teorias desenvolvidas para placas e elasticidade plana.

- A associação de macro-elementos via sub-regiões.

- Limitações à aplicação do método de sub-regiões através do MEC.

Um dos primeiros estudos que relacionam a análise de estruturas tridimensionais formadas por associação de placas foi apresentado no artigo Tanaka et al. (1998) que 
aplicou o método de elementos de contorno a problema de vibração livre de estruturas finas de placas laminadas. As soluções fundamentais estática são utilizadas para a derivação das equações integrais no plano e fora do plano. Todas as equações integrais implementadas são regularizadas até uma ordem integrável e, em seguida, foram discretizadas por meio de elementos de contorno. Todo o sistema de equações para a associação elástica de estruturas de placa finas são obtidas através da montagem das componentes satisfazendo as condições de equilíbrio e compatibilidade na interface, bem como as condições de contorno.

O trabalho de Sanches et al. (2007) é uma versão direta do método dos elementos de contorno (MEC) desenvolvido para modelar a resposta dinâmica estacionária de estruturas de placas reforçadas, tais como painéis reforçados em edifícios, automóveis e aviões. As soluções fundamentais estacionárias e dinâmicas de placas finas e estado plano de tensão são usados para transformar equações diferenciais parciais em equações integrais de contorno. Dois conjuntos de equações integrais desacoplados são formulados, respectivamente, para o estado plano (membrana) e para o estado fora do plano (flexão). Estes sistemas desacoplados são unidos para formar o macro-elemento. A associação destes macro-elementos é capaz de simular estruturas de paredes finas, incluindo estruturas de placas reforçadas.

Di Pisa et al. (2010) aplicou o método de elementos de contorno para grandes deformações de placas sob cisalhamento em associação no espaço. Cada placa é modelada como uma sub-região submetida a cargas de flexão e esforços no plano. Termos não-lineares na formulação integral devido à grandes deformações são tratados como forças de corpo. As integrais de domínio associadas são transformadas em integrais de contorno usando o método da reciprocidade dual. Derivadas dos deslocamentos no contorno surgem devido aos termos não-lineares. Seus valores são avaliadas em pontos internos do domínio usando funções de base radial. Seções da placa são unidas ao longo de suas interfaces usando as condições de compatibilidade e de equilíbrio.

Di Pisa et al. (2012) associou o método dos elementos de contorno a formulação de multiregião com objetivo de simular uma placa submetida a grandes deflexões. Funções de base radial são usadas para aproximar as derivadas dos termos de deflexão. O método da reciprocidade dual é usado para transferir todas as integrais de domínio para o contorno.

Di Pisa and Aliabadi (2013) desenvolveram um método de análise de elementos de contorno para a modelagem do crescimento de trincas em placa espessas (na formulação de placas espessas é considerado o efeito da tensão cisalhante transversal). Uma formulação dos elementos de contorno com multi-região foi implementada, enquanto o método de elementos de contorno duplo é aplicado para extrair os fatores de intensi- 
dade tensão. A propagação da trinca é modelada com novos elementos de contorno e a direção é calculada por meio do critério da máxima tensão circunferencial.

Baiz and Aliabadi (2009a) apresentaram uma formulação dos elementos de contorno com sub-regiões para a análise de instabilidades locais em placas e cascas. Cada subregião é formada pelo acoplamento dos elementos de contorno de placa deformáveis ao cisalhamento e elasticidade plana. Integrais de domínio que aparecem na formulação (devido à curvatura e devido à carga no domínio) são transformadas em integrais de contorno. Tensões de membrana em pontos discretos do domínio de cada sub-região (placa ou casca) são obtidas a partir do estado de pré-flambagem, resultando em um conjunto de equações lineares em função da deflexão e do fator de carga.

Di Pisa and Aliabadi (2015) apresentou um método dos elementos de contorno para análise de trincas em painéis aeronáuticos enrijecidos reparados por placas rebitadas ou coladas. A formulação de multi-região é utilizada para modelar os reforços. Eles são, em seguida, conectados às placas por meio de rebites. O método de elementos de contorno é usado para simular a presença de trincas. O método do deslocamentos de abertura da trinca (COD) e a integral $J$ são implementadas para calcular os fatores de intensidade de tensão.

Costa (2015) desenvolveu uma formulação do método dos elementos de contorno para a análise de estruturas formadas pela associação tridimensional de placas espessas. Nesta abordagem, são estabelecidos seis graus de liberdade por nó, três deslocamentos e três rotações desenvolvidas a partir da associação das formulações de elasticidade plana e placas deformáveis por cisalhamento. A partir destas, dois deslocamentos vem da formulação de elasticidade plana, um deslocamento e duas rotações da formulação de placas. Para que se obtenha a terceira rotação, uma equação de rotação no plano é incluída aplicando a equação integral de contorno na expressão analítica da rotação de elasticidade plana, oriunda da teoria da elasticidade. No arranjo tridimensional, cada placa é definida como uma sub-região e os termos locais são calculados. Após a necessária transformação dessas equações para um plano de referência comum, as condições de compatibilidade de deslocamentos e rotações, e equilíbrio de momentos e forças de superfície são impostas.

Como pode-se observar, em Costa (2015) houve a necessidade de uma equação de rotação no plano na associação de placas espessas e, no caso desta tese, onde foi usada placas de Kirchhoff, não ocorre este problema. Esta é uma vantagem desta formulação de placas finas sobre a de placas espessas. Esta formulação tem um número reduzido de graus de liberdade quando comparada com a formulação proposta por Costa (2015). No caso deste trabalho tem-se 4 graus de liberdade por nós, enquanto que, na formulação empregada em Costa (2015), tem-se 6 graus de liberdade por nós. Além disso, as 
soluções fundamentais de placas finas são muito mais simples que as soluções de placas deformáveis pelo cisalhamento, o que proporciona um ganho no tempo computacional.

Desta forma, a principal contribuição deste trabalho é a associação da formulação do método dos elementos de contorno de placas finas e elasticidade plana para problemas estáticos e dinâmicos onde somente o contorno é discretizado. Não foi encontrado na literatura outro trabalho onde problemas dinâmicos de associação de placas no espaço sob carregamento estático e dinâmico fossem analisados sem a discretização do domínio. Desta forma, este trabalho é original e apresenta um avanço nas formulações do método dos elementos de contorno.

\subsection{Organização dos capítulos}

Este trabalho tem como principal objetivo a análise de deslocamentos em estruturas planas formadas pela associação de placas finas no espaço sob carregamentos estáticos e dinâmicos.

No Capítulo 2 será feita uma introdução aos conceitos básicos da teoria da elasticidade linear e plana para materiais isotrópicos.

No Capítulo 3 será apresentado conceitos da teoria da elasticidade plana para materiais isotrópicos, que será empregado nas formulações matemáticas para o desenvolvimento das equações integrais de contorno. A formulação de tensão e deformação será revista, levando à definição da equação constitutiva isotrópica.

No Capítulo 4 serão apresentadas algumas considerações gerais sobre placas, incluindo as definições e nomenclatura relacionadas. As hipóteses básicas das teorias de placas de Kirchhoff serão introduzidas. Uma vez introduzidas, estas hipóteses serão relacionadas com a teoria de Kirchhoff. Com base nesta teoria, serão obtidas as equações constitutivas de placas. Será mostrado o cálculo da rigidez à flexão em direções arbitrárias. Além disso, serão relacionadas as variáveis referentes às três principais condições de contorno: livre, apoiada e engastada. Será apresentada a obtenção das equações diferenciais e das soluções fundamentais isotrópicas.

No Capítulo 5 a formulação de elementos de contorno para flexão de placas é apresentada de forma detalhada. Será apresentada a obtenção da equação integral de contorno para deslocamentos. Além disso, é descrito como calcular os termos das matrizes de influência.

No Capítulo 6 são apresentadas duas formulações para o tratamento de integrais de domínio na formulação de elementos de contorno. A primeira faz a transformação exata da integral de domínio proveniente da carga distribuída em integral de contorno. A segunda transfere os efeitos da integral de domínio para o contorno usando-se o 
método de elementos de contorno de integração radial.

No Capítulo 7 desenvolve-se o conceito de associação tri-dimensional de elementos planos utilizando a técnica de sub-regiões do MEC.

No Capítulo 8 são apresentados resultados numéricos obtidos com as rotinas implementadas para associação de placas no plano e espaço considerando problemas estáticos.

No Capítulo 9 são apresentados resultados numéricos para a formulação de elementos de contorno aplicada ao cálculo do campo de deslocamento para problemas transientes no domínio do tempo.

Por fim, no Capítulo 10 são apresentadas as considerações finais e conclusões obtidas através da análise dos resultados apresentados neste trabalho. 


\section{Capítulo 2}

\section{Teoria da Elasticidade Linear}

\subsection{Introdução}

As teorias clássicas de placas e cascas são uma importante aplicação da teoria da elasticidade, que lida com as relações de forças, deslocamentos, tensões, e deformação em um corpo elástico. Quando um corpo sólido é sujeito a forças externas, estas deforma-o, produzindo deformações e tensões internas. A deformação depende das condições de contorno do corpo, da carga aplicada, e das propriedades mecânicas do material. A teoria da elasticidade linear restringi-se a atenção para materiais lineares elásticos; isto é, as relações entre tensões e deformações são linear, e as deformações e tensões anulam-se quando as forças externas são removidas. A teoria clássica da elasticidade assume que o material é homogêneo e isotrópico, ou seja, as suas propriedades mecânicas são as mesmas em todas as direções e em todos os pontos.

A presente seção contém uma breve descrição da teoria da elasticidade linear e plana que será útil para o desenvolvimento da teoria de placa.

\subsection{Tensão: Tensor de tensão}

Considere um corpo elástico sujeito a cargas externas que estão em equilíbrio. Em seguida, considere um ponto material em qualquer lugar no interior do corpo. Se atribuirmos um sistema de coordenadas cartesianas com eixos $x_{1}, x_{2}, x_{3}$, como mostrado na Figura 2.1; é conveniente atribuir um elemento infinitesimal em forma de paralelepípedo $d x_{1}, d x_{2}, d x_{3}$, com faces paralelas aos planos coordenados. Para efeitos de quantificação do modo como ocorre a transmissão de forças no interior de um sólido é necessário introduzir a noção de tensão, como foi definida por Cauchy.

As tensões atuando na face do elemento infinitesimal descrevem a intensidade das forças internas em um ponto da face do sólido. Estas tensões podem ser divididas 


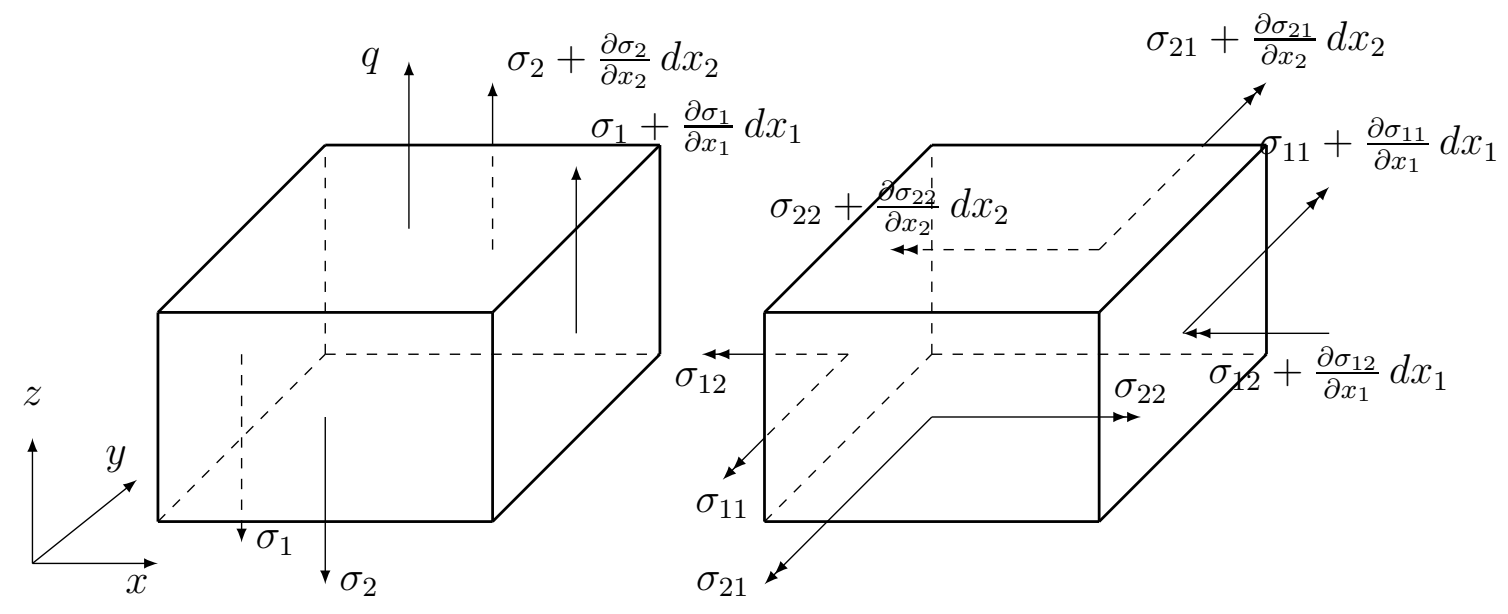

Figura 2.1: Estado geral de tensão.

em uma componente normal (tensão normal) e uma componente tangente (tensão de cisalhamento) à face. Como resultado, as três componentes da tensão, definidas por $\sigma_{11}, \sigma_{12}, \sigma_{13}$ para a face 1 , atuarão sobre cada elemento. A notação subscrita pelos componentes de tensão é interpretada da seguinte forma: o primeiro índice indica o sentido de uma normal exterior à face na qual atua a componente de tensão; o segundo subscrito refere-se ao sentido da própria tensão.

Quando a posição relativa entre dois pontos quaisquer num corpo contínuo é alterada, é dito que o corpo sofreu deformação. Se a distância entre qualquer par de pontos no corpo permanece constante durante o movimento, o corpo é dito rígido. $\mathrm{O}$ movimento de corpo rígido é caracterizado apenas pela translação e rotação.

Em mecânica do contínuo, o tensor tensão de Cauchy $\sigma$, tensor tensão verdadeira ou simplesmente denominado tensor tensão, denominado em memória de AugustinLouis Cauchy, é um tensor de segunda ordem, com nove componentes $\sigma_{i j}$, que define completamente o estado de tensão em um ponto no domínio de um material em sua configuração deformada. O tensor relaciona um vetor diretor de comprimento unitário $\mathbf{n}$ com o vetor tensão $T^{\mathbf{n}}$ sobre uma superfície imaginária perpendicular a $\mathbf{n}$ :

$$
T^{(\mathbf{n})}=\mathbf{n} \cdot \sigma \quad T_{j}^{(n)}=\sigma_{i j} \cdot n_{i} .
$$

Para os eixos coordenados da Figura 2.1, usando a notação indicial,

$$
\mathbf{T}_{\mathbf{S}}=\left[\begin{array}{ccc}
\sigma_{x} & \tau_{x y} & \tau_{x z} \\
\tau_{y x} & \sigma_{y} & \tau_{y z} \\
\tau_{z x} & \tau_{z y} & \sigma_{z}
\end{array}\right]
$$

o qual é simétrico em relação à diagonal principal devido a lei da reciprocidade das tensões de cisalhamento, 


$$
\tau_{x y}=\tau_{y x} ; \quad \tau_{x z}=\tau_{z x} \quad \tau_{y z}=\tau_{z y}
$$

Assim, apenas seis das nove componentes de tensão no tensor expresso pela Equação 2.2 são independentes. O tensor de tensão, $\mathbf{T}_{S}$, caracteriza completamente o estado tridimensional de tensão em um ponto do corpo sólido.

Para a análise de tensão de placas elásticas no estado plano, $\sigma_{z}=\tau_{y z}=\tau_{x z}=0$. Assim, a Equação (2.2) pode ser simplificada por:

$$
\mathbf{T}_{\mathbf{S}}=\left[\begin{array}{cc}
\sigma_{x} & \tau_{x y} \\
\tau_{y x} & \sigma_{y}
\end{array}\right], \quad \text { onde } \tau_{x y}=\tau_{y x}
$$

\subsection{Relações Deformação - Deslocamento}

Observemos o corpo elástico mostrado na Figura 2.2, é suportada de tal maneira que os deslocamentos do corpo rígido (translações e rotações) são evitadas. Assim, este corpo deforma sob a ação de forças exteriores e cada um dos pontos tem pequenos deslocamentos elásticos. Por exemplo, um ponto $A$ tinha as coordenadas $x, y, z$ no estado inicial não deformado. Após a deformação, esse ponto move-se para posição $A^{*}$ e suas coordenadas tornam-se $x^{\prime}=x+u, y^{\prime}=y+v, z^{\prime}=z+w$, onde $u, v$ e $w$ são as projeções do vetor deslocamento do ponto $A$, vetor $A A^{\prime} \operatorname{nos}$ eixos coordenados $x, y$ e $z$. No caso geral, $u, v$ e $w$ são funções de $x, y$ e $z$.

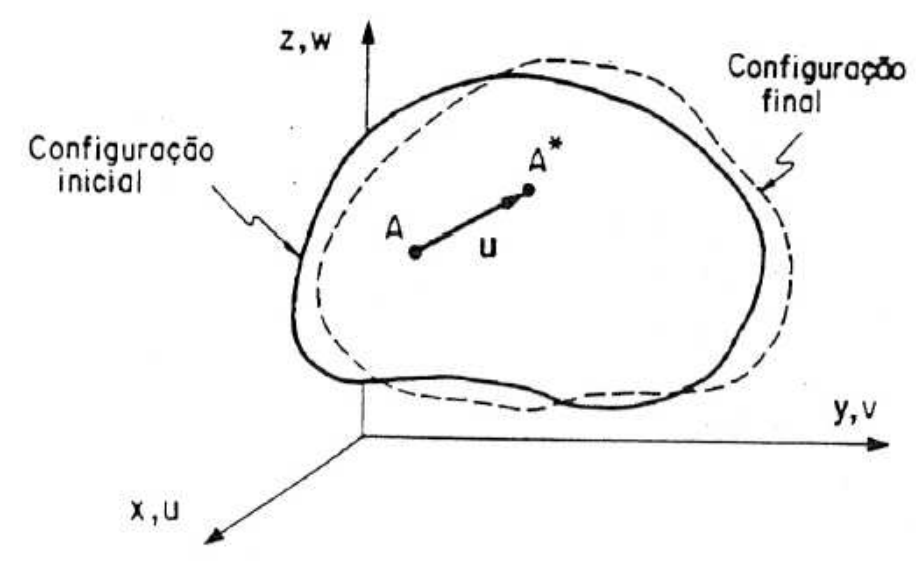

Figura 2.2: Deformações: campo de deslocamentos.

As deformações decorrentes podem ser indicadas por meio de índices. Dessa forma, as deformações lineares são:

$$
\epsilon_{x}=\frac{\delta(d x)}{d x} ; \quad \epsilon_{y}=\frac{\delta(d y)}{d y} \quad \epsilon_{z}=\frac{\delta(d z)}{d z}
$$


estas equações são chamadas de deformações lineares numa direção qualquer. Na Equação (2.5), o termo $\delta(d x)$, pode ser expresso segundo series de Taylor, ou seja, $\delta(d x)=\frac{\partial u}{\partial x} d x$, etc; então, pode-se escrever

$$
\epsilon_{x}=\frac{\partial u}{\partial x}, \quad \epsilon_{y}=\frac{\partial v}{\partial y}, \quad \epsilon_{z}=\frac{\partial w}{\partial z} .
$$

A deformação angular associada a um par de direções ortogonais quaisquer no ponto $A$ são denominadas por $\gamma_{x y}, \gamma_{x z}$ e $\gamma_{y z}$. O ângulo $B A C$ na Figura 2.3, apresenta configuração (deformada) $B^{*} A^{*} C^{*}$,assim, a deformação angular em função do plano de deslocamentos são:

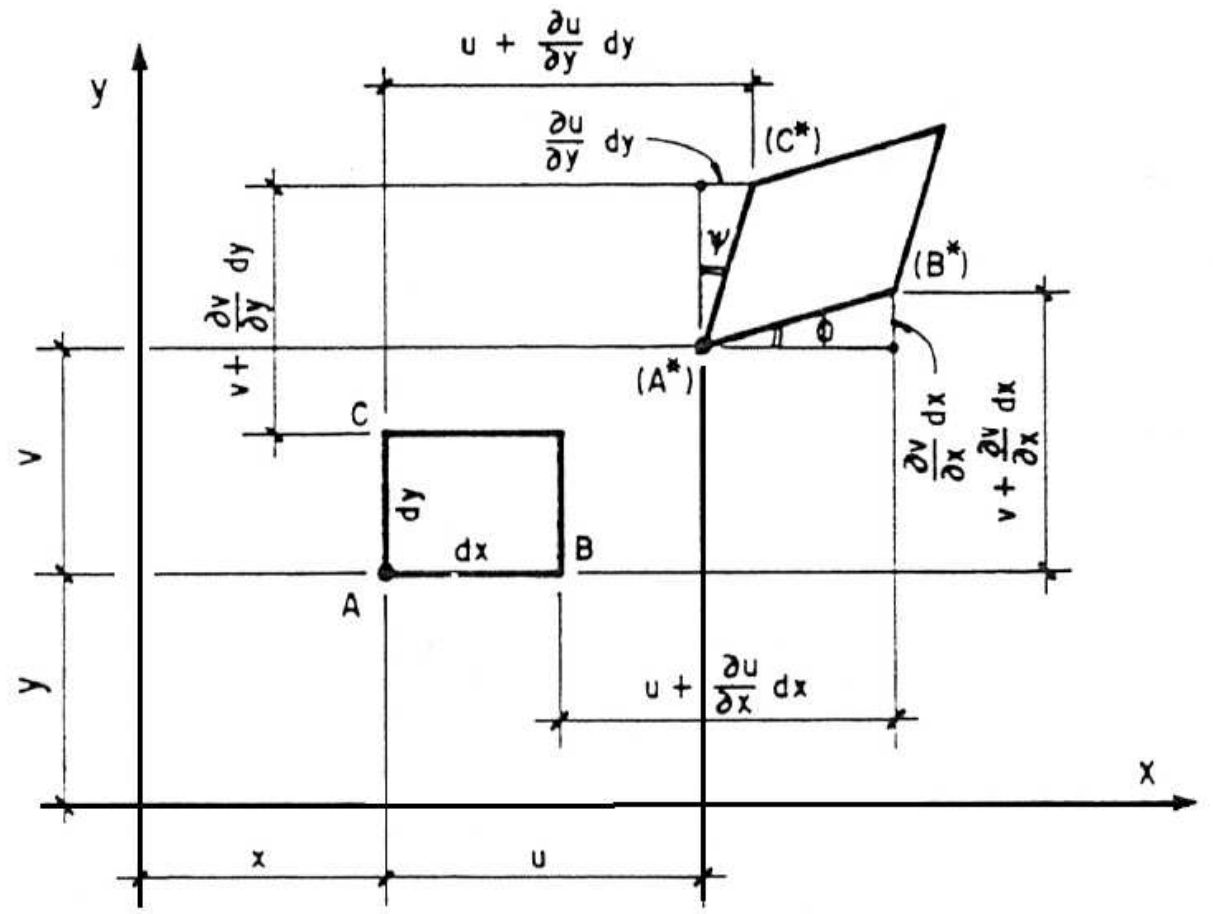

Figura 2.3: Configuração deforma de um corpo

$$
\gamma_{x y}=\frac{\frac{\partial v}{\partial x} d x}{d x+\frac{\partial u}{\partial x} d x}+\frac{\frac{\partial u}{\partial y} d y}{d y+\frac{\partial v}{\partial y} d y}=\frac{\frac{\partial v}{\partial x}}{1+\frac{\partial u}{\partial x}}+\frac{\frac{\partial u}{\partial y}}{1+\frac{\partial v}{\partial y}},
$$

Limitando-se ao caso de pequenas deformações, pode-se omitir os termos $\frac{\partial u}{\partial x}$ e $\frac{\partial v}{\partial y}$ do denominador da expressão acima, obtendo-se

$$
\gamma_{x y}=\frac{\partial v}{\partial x}+\frac{\partial u}{\partial y}
$$

Similarmente, pode-se obter $\gamma_{x z}$ e $\gamma_{y z}$. A deformação angular é dada por:

$$
\gamma_{x y}=\frac{\partial u}{\partial y}+\frac{\partial v}{\partial x}, \quad \gamma_{x z}=\frac{\partial u}{\partial z}+\frac{\partial w}{\partial x}, \quad \gamma_{y z}=\frac{\partial v}{\partial z}+\frac{\partial w}{\partial y}
$$


Assim, o tensor de tensão observado na Equação (2.2), pode ser definido como tensor de deformação

$$
\mathbf{T}_{\mathbf{D}}=\left[\begin{array}{ccc}
\epsilon_{x} & \frac{1}{2} \gamma_{x y} & \frac{1}{2} \gamma_{x z} \\
\frac{1}{2} \gamma_{y x} & \epsilon_{y} & \frac{1}{2} \gamma_{y z} \\
\frac{1}{2} \gamma_{z x} & \frac{1}{2} \gamma_{z y} & \epsilon_{z}
\end{array}\right] .
$$

Sabendo-se que, o tensor de deformação é simétrico, tem-se:

$$
\varepsilon_{x y}=\varepsilon_{y x}, \quad \varepsilon_{x z}=\varepsilon_{z x}, \quad \varepsilon_{y z}=\varepsilon_{z y}
$$

\subsection{Equações constitutivas}

Em elasticidade linear, o vetor de deslocamentos e suas derivadas são assumidos como infinitesimais. O tensor de deformação, considerando deslocamentos infinitesimais, pode ser escrito como:

$$
\varepsilon_{x}=\frac{1}{E}\left[\sigma_{x}-\nu\left(\sigma_{y}+\sigma_{z}\right)\right], \quad \varepsilon_{y}=\frac{1}{E}\left[\sigma_{y}-\nu\left(\sigma_{x}+\sigma_{z}\right)\right], \quad \varepsilon_{z}=\frac{1}{2}\left[\sigma_{z}-\nu\left(\sigma_{y}+\sigma_{x}\right)\right],
$$

$\mathrm{e}$

$$
\gamma_{x y}=\frac{1}{G} \tau_{x y}, \quad \gamma_{x z}=\frac{1}{G} \tau_{x z}, \quad \gamma_{y z}=\frac{1}{G} \tau_{y z},
$$

onde $E, \nu$ e $G$ são os módulos de elasticidade, coeficiente de Poisson e módulo de cisalhamento. A relação entre $E$ e $G$ é dada por:

$$
G=\frac{E}{2(1+\nu)}
$$

\subsection{Equações de equilíbrio}

As componentes de tensão introduzidas acima, devem satisfazer as equações de equilíbrio:

$$
\begin{aligned}
& \frac{\partial \sigma_{x}}{\partial x}+\frac{\partial \tau_{x y}}{\partial y}+\frac{\partial \tau_{x z}}{\partial z}+b_{x}=0 \\
& \frac{\partial \sigma_{y}}{\partial y}+\frac{\partial \tau_{y x}}{\partial x}+\frac{\partial \tau_{y z}}{\partial z}+b_{y}=0 \\
& \frac{\partial \sigma_{z}}{\partial z}+\frac{\partial \tau_{z x}}{\partial x}+\frac{\partial \tau_{z y}}{\partial y}+b_{z}=0
\end{aligned}
$$


onde $b_{x}, b_{y}$ e $b_{z}$ são as forças de corpo. A Equação 2.15 pode ser escrita como:

$$
\sigma_{i j, j}+b_{i}=0
$$

\subsection{Equações compatibilidade}

Considerando que é necessário conhecer o valor das seis componentes do tensor de deformação, Equação (2.9), a Equação (2.10), contém apenas três componentes de deslocamentos: $u_{x}, u_{y}, u_{z}$. Assim, este sistema de equações não possui uma solução única, pelo que as componentes não podem ser independentes entre si. Estas equações adicionais são definidas como equações de compatibilidade. Eliminando-se as componentes de deslocamento por sucessivas diferenciações, as seguintes equações de compatibilidade são obtidas

$$
\begin{aligned}
& \frac{\partial^{2} \varepsilon_{x}}{\partial y^{2}}+\frac{\partial^{2} \varepsilon_{y}}{\partial x^{2}}=\frac{\partial^{2} \gamma_{x y}}{\partial x \partial y} \\
& \frac{\partial^{2} \varepsilon_{y}}{\partial z^{2}}+\frac{\partial^{2} \varepsilon_{z}}{\partial y^{2}}=\frac{\partial^{2} \gamma_{y z}}{\partial y \partial z} \\
& \frac{\partial^{2} \varepsilon_{z}}{\partial x^{2}}+\frac{\partial^{2} \varepsilon_{x}}{\partial z^{2}}=\frac{\partial^{2} \gamma_{x z}}{\partial x \partial z}
\end{aligned}
$$

$\mathrm{e}$

$$
\begin{aligned}
& \frac{\partial}{\partial z}\left[\frac{\partial \gamma_{y z}}{\partial x}+\frac{\partial \gamma_{x z}}{\partial y}-\frac{\partial \gamma_{x y}}{\partial z}\right]=2 \frac{\partial^{2} \varepsilon_{z}}{\partial x \partial y} \\
& \frac{\partial}{\partial x}\left[\frac{\partial \gamma_{x z}}{\partial y}+\frac{\partial \gamma_{x y}}{\partial z}-\frac{\partial \gamma_{y z}}{\partial x}\right]=2 \frac{\partial^{2} \varepsilon_{x}}{\partial y \partial z} \\
& \frac{\partial}{\partial y}\left[\frac{\partial \gamma_{x y}}{\partial z}+\frac{\partial \gamma_{y z}}{\partial x}-\frac{\partial \gamma_{x z}}{\partial y}\right]=2 \frac{\partial^{2} \varepsilon_{y}}{\partial x \partial z}
\end{aligned}
$$

Para o estado plano de tensão $\left(\sigma_{z}=0, \tau_{x z}=\tau_{y z}=0\right)$, as condições de equilíbrio dada nas Equações (2.15) resulta em:

$$
\begin{aligned}
& \frac{\partial \sigma_{x}}{\partial x}+\frac{\partial \tau_{x y}}{\partial y}+b_{x}=0 \\
& \frac{\partial \sigma_{y}}{\partial y}+\frac{\partial \tau_{y x}}{\partial x}+b_{y}=0
\end{aligned}
$$

e as equações de compatibilidade 


$$
\frac{\partial^{2} \varepsilon_{x}}{\partial y^{2}}+\frac{\partial^{2} \varepsilon_{y}}{\partial x^{2}}=\frac{\partial^{2} \gamma_{x y}}{\partial x \partial y} \quad\left(\gamma_{x z}=\gamma_{y z}=\varepsilon_{z}=0\right)
$$

A Equação (2.20) pode ser escrita de forma mais compacta usando a notação indicial. Obtêm-se:

$$
\left(\frac{\partial^{2}}{\partial x^{2}}+\frac{\partial^{2}}{\partial y^{2}}\right)\left(\sigma_{x}+\sigma_{y}\right)=0
$$

Essas equações são chamadas de equações de Levy's. Indroduzindo as funções de tensão de Airy's $\phi(x, y)$ que satisfaz

$$
\sigma_{x}=\frac{\partial^{2} \phi}{\partial y^{2}}, \quad \sigma_{y}=\frac{\partial^{2} \phi}{\partial x^{2}}, \quad \tau_{x y}=-\frac{\partial^{2} \phi}{\partial x \partial y} .
$$

A Equação (2.21), torna-se

$$
\nabla^{2} \nabla^{2} \phi=0
$$

onde

$$
\nabla^{2}=\frac{\partial^{2}}{\partial x^{2}}+\frac{\partial^{2}}{\partial y^{2}}
$$

representa a equação de Laplace bidimensional. 


\section{Capítulo 3}

\section{Método dos Elementos de Contorno para elasticidade plana}

Esta seção apresenta as equações de equilíbrio da formulação de elasticidade plana isotrópica, a identidade Somigliana que permite obter os valores dos deslocamentos e o tipo de elemento de contorno utilizado neste trabalho. As formulações matemáticas presentes neste capítulo serão usados posteriormente, quer seja, na obtenção da formulação do método dos elementos de contorno, quer seja, na comparação com resultados numéricos obtidos a partir das formulações propostas.

\subsection{Formulação integral de contorno para elasticidade}

Entre as várias possibilidades de obtenção das equações integrais de contorno para elasticidade, podemos utilizar o teorema da reciprocidade de Betti. A formulação é obtida a partir deste teorema, considerando dois estados equilibrados, um no domínio do problema e o outro naquele conhecido como espaço fundamental, com variáveis $\left(u_{i}, t_{i}, b_{i}\right)$ e $\left(u_{i}^{*}, t_{i}^{*}, b_{i}^{*}\right)$, respectivamente, onde $u_{i}$ e $u_{i}^{*}$ são os deslocamentos, $t_{i}$ e $t_{i}^{*}$ são as forças de superfície, e $b_{i}$ e $b_{i}^{*}$ são as forças de corpo.

Assumindo-se uma função vetorial contínua $u_{i}^{*}$, que representa o deslocamento de um estado elasto-estático definido sobre um domínio $\Omega$, como sendo uma função peso da equação de equilíbrio (2.19), tem-se:

$$
\int_{\Omega} \sigma_{i j, j} u_{i}^{*} d \Omega+\int_{\Omega} b_{i} u_{i}^{*} d \Omega=0
$$

Pela regra de derivação do produto de duas funções tem-se:

$$
\left(\sigma_{i j} u_{i}^{*}\right)_{, j}=\sigma_{i j, j} u_{i}^{*}+\sigma_{i j} u_{i, j}^{*}
$$


Pode-se escrever $u_{i, j}^{*}$ como a soma de um tensor simétrico e um anti-simétrico, da forma

$$
u_{i, j}^{*}=\frac{1}{2}\left(u_{i, j}^{*}+u_{j, i}^{*}\right)+\frac{1}{2}\left(u_{i, j}^{*}-u_{j, i}^{*}\right)=\varepsilon_{i j}^{*}+\omega_{i j}^{*}
$$

sendo que $\varepsilon_{i j}^{*}$ e $\omega_{i j}^{*}$ representam os tensores deformação (simétrico) e de corpo rígido (anti-simétrico), respectivamente, do estado elástico " * ".

Substituindo (3.3) em (3.2) tem-se

$$
\left(\sigma_{i j} u_{i}^{*}\right)_{, j}=\sigma_{i j, j} u_{i}^{*}+\sigma_{i j} \varepsilon_{i j}^{*}+\sigma_{i j} \omega_{i j}^{*}
$$

sendo $\sigma_{i j}$ um tensor simétrico. O produto de um tensor simétrico por um anti-simétrico é nulo. Desta forma, a equação (3.4) torna-se

$$
\sigma_{i j, j} u_{i}^{*}=\left(\sigma_{i j} u_{i}^{*}\right)_{, j}-\sigma_{i j} \varepsilon_{i j}^{*}
$$

Substituindo a equação (3.5) na equação (3.1) tem-se

$$
-\int_{\Omega} \sigma_{i j} \varepsilon_{i j}^{*} d \Omega+\int_{\Omega}\left(\sigma_{i j} u_{i}^{*}\right)_{, j} d \Omega+\int_{\Omega} b_{i} u_{i}^{*} d \Omega=0
$$

Pelo teorema de Green tem-se:

$$
\int_{\Omega}\left(\sigma_{i j} u_{i}^{*}\right)_{, j} d \Omega=\int_{\Gamma}\left(\sigma_{i j} u_{i}^{*}\right) n_{j} d \Gamma=\int_{\Gamma} t_{i} u_{i}^{*} d \Gamma
$$

onde

$$
t_{i}=\sigma_{i j} n_{j}
$$

Substituindo (3.7) em (3.6), tem-se

$$
\int_{\Omega} \sigma_{i j} \varepsilon_{i j}^{*} d \Omega=\int_{\Gamma} t_{i} u_{i}^{*} d \Gamma+\int_{\Omega} b_{i} u_{i}^{*} d \Omega
$$

Se partirmos da equação (2.16) como sendo a correspondente ao estado $u_{i}^{*}$ e a função de interpolação da equação (3.1) como sendo $u_{i}$, obtém-se, de forma análoga a anterior

$$
\int_{\Omega} \sigma_{i j}^{*} \varepsilon_{i j} d \Omega=\int_{\Gamma} t_{i}^{*} u_{i} d \Gamma+\int_{\Omega} b_{i}^{*} u_{i} d \Omega
$$

Pelo Teorema Betti dois estados de um mesmo material podem ser relacionados por $\sigma_{i j}^{*} \varepsilon_{i j}=\sigma_{i j} \varepsilon_{i j}^{*}$. Desta forma, igualando-se as equações (3.10) e (3.9), tem-se

$$
\int_{\Gamma} t_{i} u_{i}^{*} d \Gamma+\int_{\Omega} u_{i}^{*} b_{i} d \Omega=\int_{\Gamma} t_{i}^{*} u_{i} d \Gamma+\int_{\Omega} u_{i} b_{i}^{*} d \Omega
$$


A equação integral (3.11) relaciona dois estados quaisquer de tensões. Para que se possa tratar problemas de elasticidade em meio contínuo, será adotado que um destes estados é conhecido, e o outro se deseja determinar. No caso de elementos de contorno, o estado conhecido é o chamado estado fundamental que corresponde a resposta de um corpo infinito sujeito a uma carga concentrada unitária em um ponto $\mathbf{x}^{\prime}$. A representação matemática de uma carga concentrada unitária é dada pelo delta de Dirac, que é definido como

$$
\begin{cases}\delta\left(\mathbf{x}-\mathbf{x}^{\prime}\right)=\infty & \text { se } \mathbf{x}=\mathbf{x}^{\prime} \\ \delta\left(\mathbf{x}-\mathbf{x}^{\prime}\right)=0 & \text { se } \mathbf{x} \neq \mathbf{x}^{\prime} \\ \int_{-\infty}^{\infty} \delta\left(\mathbf{x}-\mathbf{x}^{\prime}\right) d \Omega=1 & \end{cases}
$$

A razão da escolha do estado fundamental deve-se ao fato que a função delta de Dirac reduz o número de integrais de domínio, pois esta possui a propriedade

$$
\int_{\Omega} f(\mathbf{x}) \delta\left(\mathbf{x}-\mathbf{x}^{\prime}\right) d \Omega=f\left(\mathbf{x}^{\prime}\right)
$$

para um dado ponto $\mathbf{x}^{\prime} \in \Omega$.

Considerando o estado "* " como sendo o estado fundamental de um problema estático livre de forças de corpo $\left(b_{i}^{*}=0\right)$, a equação (3.11) pode ser escrita como

$$
\int_{\Gamma} T_{i k} u_{i} d \Gamma+\int_{\Omega} b_{i} U_{i k} d \Omega=\int_{\Gamma} t_{i} U_{i k} d \Gamma-\int_{\Omega} \delta_{i k} u_{i} d \Omega
$$

onde $U_{i k}$ e $T_{i k}$ representam respectivamente deslocamentos e forças de superfície na direção $k$, num ponto $\mathbf{x}$, devido a uma força concentrada unitária aplicada de forma estática num ponto $\mathbf{x}^{\prime}$ numa direção $i$. Por serem soluções do estado fundamental, $U_{i k}$ e $T_{i k}$ são chamadas soluções fundamentais de deslocamentos e forças de superfície, respectivamente.

Devido a propriedade (3.13), a equação (3.14) pode ser escrita como

$$
u_{k}+\int_{\Gamma} T_{i k} u_{i} d \Gamma=\int_{\Gamma} U_{i k} t_{i} d \Gamma-\int_{\Omega} b_{i} U_{i k} d \Omega
$$

Esta equação também é conhecida como Identidade de Somigliana e permite obter os valores dos deslocamentos $u$ em qualquer ponto $P$ do domínio $\Omega$ do problema, considerando-se que os valores de deslocamentos $u_{i}$ e forças de superfície $t_{i}$ no contorno da geometria e as forças de corpo $b_{i}$ são conhecidos.

Considerando que as forças de corpo $b_{i}$ são nulas, pode-se escrever:

$$
u_{k}+\int_{\Gamma} T_{i k} u_{i} d \Gamma=\int_{\Gamma} U_{i k} t_{i} d \Gamma
$$




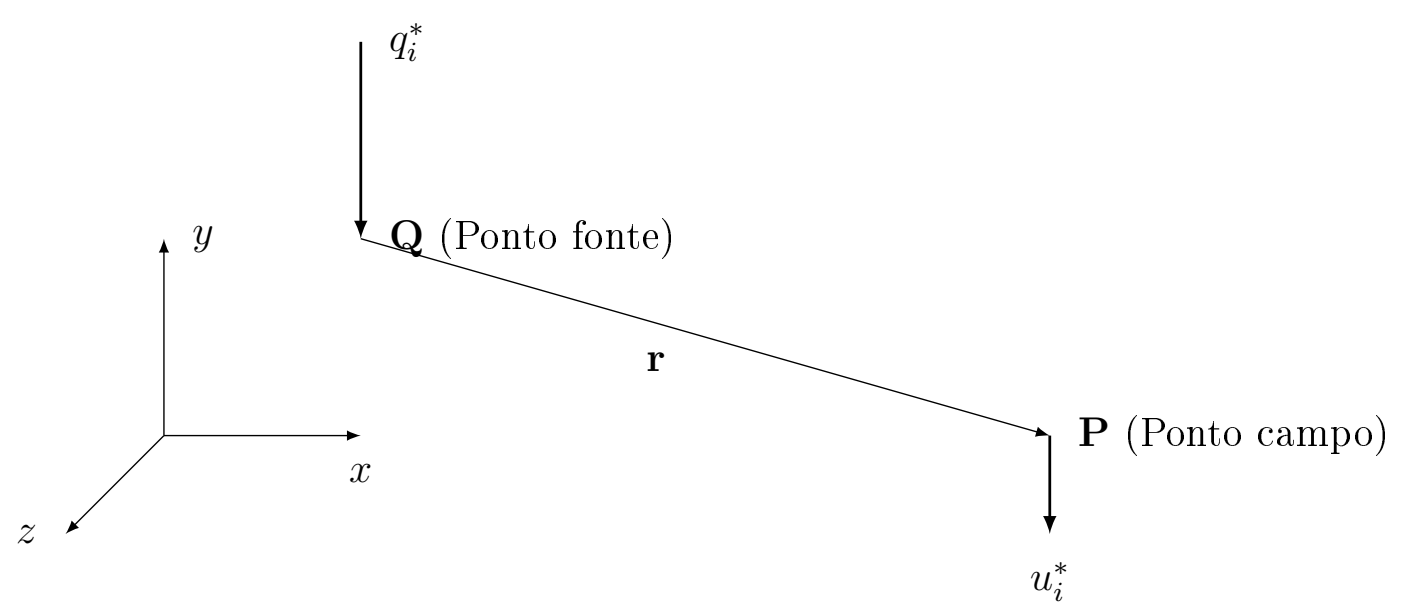

Figura 3.1: Ponto de carregamento (Q) e de deslocamento (P).

\subsection{Soluções fundamentais}

Para um estado plano de tensões as soluções fundamentais, conforme Figura 3.1 para os deslocamentos e forças de superfícies são dadas, respectivamente, por:

$$
\begin{gathered}
U_{i j}(X, x)=\frac{1}{8 \pi \mu}(1-\nu)(3-4 \nu) \log \frac{1}{R} \delta_{i j}+R_{, i} R_{, j} \\
T_{i j}(X, x)=\frac{-1}{4 \pi(1-\nu) R}\left[(1-2 \nu) \delta_{i j}+2 R_{, i} R_{, j}\right] \frac{\partial R}{\partial n}-(1-2 \nu)\left(R_{, i} n_{j}-R_{, j} n_{i}\right)
\end{gathered}
$$

Note que tanto a solução fundamental de deslocamentos quanto a de forças de superfície são singulares quando o ponto fonte $Q$ tende ao ponto campo $P$. No caso da solução fundamental de deslocamentos a singularidade é fraca (ln $r$ ). Já no caso da solução fundamental de forças de superfície tem-se uma singularidade forte $(1 / r)$, conforme Brebbia and Dominguez (1989). As formas como estas singularidades serão tratadas é mostrada na seção abaixo.

\subsection{Equações integrais singulares}

A equação integral (3.16) foi escrita para um ponto do interior do domínio. Uma vez que o ponto fonte é interno, a equação contém apenas integrandos regulares. Considere agora o limite da transição quando o ponto fonte tende ao contorno. Esta operação pode ser implementada colocando o ponto fonte no contorno e diminuindo o domínio do problema por uma região semi-circular, com contorno $\Gamma_{\epsilon}^{*}$ e raio $\epsilon$, centrado no ponto fonte, conforme mostrado na Figura 3.2. Com esta configuração, o contorno completo é dividido em duas partes, na forma 


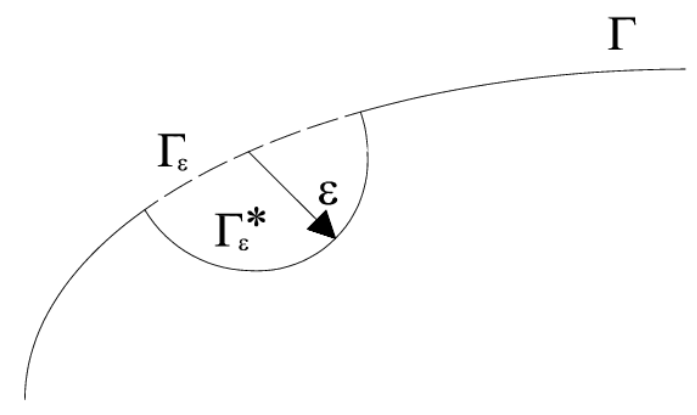

Figura 3.2: Ponto fonte localizado no contorno, circundado por uma região semicircular.

$$
\Gamma=\lim _{\epsilon \rightarrow 0}\left(\Gamma-\Gamma_{\epsilon}+\Gamma_{\epsilon}^{*}\right)
$$

onde $\epsilon$ é o raio do semi-círculo de centro no ponto fonte, pertencendo ao contorno $\Gamma$ (Figura 3.2). A equação (3.16) é, então, reescrita como:

$$
u_{l}+\lim _{\epsilon \rightarrow 0} \int_{\Gamma-\Gamma_{\epsilon}+\Gamma_{\epsilon}^{*}} T_{l i} u_{i} d \Gamma=\lim _{\epsilon \rightarrow 0} \int_{\Gamma-\Gamma_{\epsilon}+\Gamma_{\epsilon}^{*}} U_{l i} t_{i} d \Gamma
$$

A integral do lado direito da equação (3.20) contém um integrando de singularidade fraca da ordem $\ln (1 / r)$ e é integrável como uma integral imprópria. A integral do lado esquerdo tem uma singularidade forte, de ordem $1 / r$, que pode ser regularizada com o primeiro termo da expansão de Taylor em torno do ponto fonte, ou seja

$$
\begin{aligned}
\lim _{\epsilon \rightarrow 0} \int_{\Gamma-\Gamma_{\epsilon}+\Gamma_{\epsilon}^{*}} T_{l i} u_{i}(\mathbf{z}) d \Gamma= & \lim _{\epsilon \rightarrow 0} \int_{\Gamma_{\epsilon}^{*}} T_{l i}\left[u_{i}(\mathbf{z})-u_{i}\left(\mathbf{z}^{\prime}\right)\right] d \Gamma+ \\
& u_{i}\left(\mathbf{z}^{\prime}\right) \lim _{\epsilon \rightarrow 0} \int_{\Gamma_{\epsilon}^{*}} T_{l i} d \Gamma+ \\
& \lim _{\epsilon \rightarrow 0} \int_{\Gamma-\Gamma_{\epsilon}} T_{l i} u_{i}(\mathbf{z}) d \Gamma
\end{aligned}
$$

Assumindo que os deslocamentos são contínuos no ponto fonte, o primeiro termo do lado direito da equação (3.21) é integrável e desaparece no processo de limite. O segundo termo da equação representa um salto nos deslocamentos dado por $A_{i j}\left(\mathbf{z}^{\prime}\right) u_{j}\left(\mathbf{z}^{\prime}\right)$, no qual $A_{i j}\left(\mathbf{z}^{\prime}\right)$ é uma constante que depende da geometria local e das constantes elásticas. Finalmente, o terceiro termo do lado direito da equação resulta numa integral 
imprópria que é calculada no sentido do valor principal de Cauchy. Portanto, quando $\epsilon \rightarrow 0$, o ponto fonte tende ao contorno e, no limite, a equação (3.20) pode ser escrita na forma

$$
c_{l i} u_{i}+\int-T_{l i} u_{i} d \Gamma=\int_{\Gamma} U_{l i} t_{i} d \Gamma
$$

onde $f$ representa integral no sentido do valor principal de Cauchy e o coeficiente $c_{l i}\left(\mathbf{z}^{\prime}\right)$ é dado por $\delta i j+A_{i j}\left(\mathbf{z}^{\prime}\right)$, no qual $\delta_{i j}$ representa o delta de Kronecker.

\subsection{Elementos de contorno constante}

A discretização de um contorno $\Gamma$, é feita dividindo-se este em $N$ elementos geométricos de aproximação, denominados elementos de contorno, que percorrem todo ele, procurando reproduzi-lo matematicamente de forma aproximada.

Define-se elemento de contorno, ao ente geométrico unitário que possui forma e funcionalidade definida por meio de seus nós geométricos.

Os nós geométricos são formados pelo conjunto de pontos de localização do elemento, que define a forma geométrica do elemento. Estes são representados graficamente por um ".", conforme mostra a Figura 3.3. Os elementos de contorno podem possuir basicamente duas formas geométricas: a linear e a curva. Os elementos curvos podem ser de geometria parabólica, cúbica, etc.

Os nós funcionais são formados pelo conjunto de pontos pertencentes ao elemento localizados nesses nós. Eles são representados geometricamente por um "•", conforme mostra a Figura 3.3. Estes nós podem ou não coincidir com os nós geométricos.

Cada elemento possui certo número de nós geométricos e funcionais que dependem das funções de aproximação escolhidas ou utilizada para representar a geometria e a funcionalidade do elemento. O caso isoparamétrico é definido quando os nós geométricos coincidem com os nós funcionais. As funções de forma são funções de aproximação para a geometria do contorno.

Para se discretizar um contorno em elementos constantes, utiliza-se duas funções de interpolação linear para a geometria. Estas funções são dadas por:

$$
\phi_{a}(\eta)=\frac{1-\eta}{2}
$$

e

$$
\phi_{b}(\eta)=\frac{1+\eta}{2}
$$




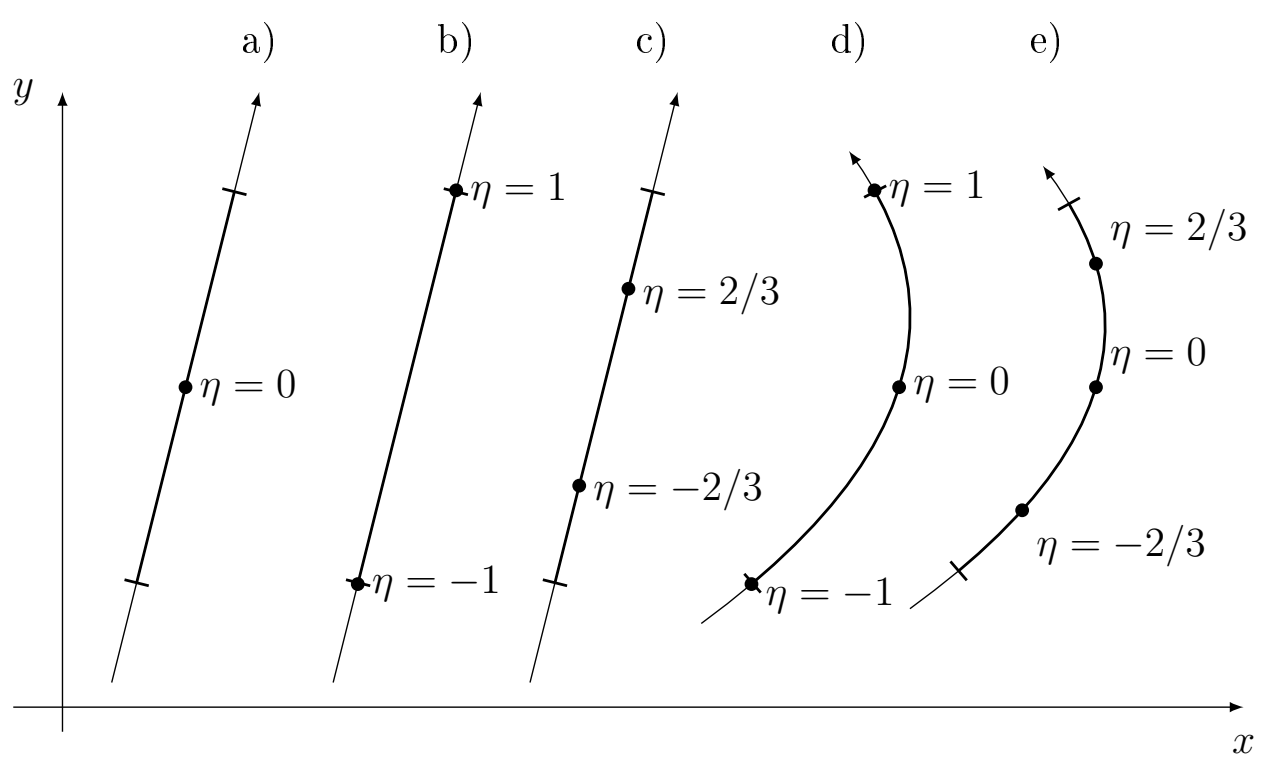

Figura 3.3: Ilustração de cinco tipos de elementos: a) constante b)lineares contínuos, c) lineares descontínuos, d) quadráticos contínuos, e) quadráticos descontínuos.

As coordenadas $x$ e $y$ dos pontos sobre o elemento são dadas por:

$$
x=x_{a} \phi_{a}(\eta)+x_{b} \phi_{b}(\eta),
$$

$\mathrm{e}$

$$
y=y_{a} \phi_{a}(\eta)+y_{b} \phi_{b}(\eta),
$$

onde $x_{a}, x_{b}, y_{a}$ e $y_{b}$ são as coordenadas dos nós físicos do elemento.

Substituindo as Equações (3.23) e (3.24) nas Equações (3.25) e (3.26), tem-se:

$$
x=x_{a} \frac{(1-\eta)}{2}+x_{b} \frac{(1+\eta)}{2},
$$

$\mathrm{e}$

$$
y=y_{a} \frac{(1-\eta)}{2}+y_{b} \frac{(1+\eta)}{2},
$$

$\mathrm{Ou}$

$$
x=\frac{\left(x_{a}+x_{b}\right)}{2}+\frac{\left(x_{b}-x_{a}\right)}{2} \eta,
$$




$$
y=\frac{\left(y_{a}+y_{b}\right)}{2}+\frac{\left(y_{b}-y_{a}\right)}{2} \eta,
$$

Chamando de:

$$
\xi_{x}=\frac{\left(x_{a}+x_{b}\right)}{2},
$$

e

$$
\xi_{y}=\frac{\left(y_{a}+y_{b}\right)}{2},
$$

Observando-se que:

$$
l_{x}=\frac{\left(x_{b}-x_{a}\right)}{2},
$$

$\mathrm{e}$

$$
l_{y}=\frac{\left(y_{b}-y_{a}\right)}{2},
$$

obtém-se:

$$
x=\xi_{x}+\left(l_{x}\right) \eta,
$$

e

$$
y=\xi_{y}+\left(l_{y}\right) \eta,
$$

\subsection{Formulação dos Elementos de Contorno Discreti- zada}

Para se obter a solução do problema elasto-estático, o contorno é dividido em elementos de contorno. Nesta etapa do trabalho, serão utilizados apenas elementos constantes (1 nó por elemento). 
Nesta formulação será mais conveniente trabalhar com vetores que usar notação indicial. Desta forma, tem-se:

$$
\begin{aligned}
& \mathbf{u}=\mathbf{u}^{(i)}, \\
& \mathbf{t}=\mathbf{t}^{(i)},
\end{aligned}
$$

sendo que as variáveis em negrito representam vetores de dimensões $2 N$, onde $N$ é o número de nós, $\mathbf{u}^{(i)}$ e $\mathbf{t}^{(i)}$ representam os valores nodais dos deslocamentos e forças de superfícies, respectivamente, $\mathbf{u}$ e $\mathbf{t}$ representam os deslocamentos e tensões ao longo do elemento, respectivamente. Neste trabalho, u e t serão considerados constantes ao longo do elemento.

Considere que o contorno tenha sido dividido em $N E$ elementos de contorno constantes. Substituindo as Equações (3.37) na Equação (3.22), tem-se

$$
\frac{1}{2} \mathbf{u}^{l}+\sum_{j=1}^{N E}\left\{f_{\Gamma_{j}} \mathbf{T} \mathbf{d} \boldsymbol{\Gamma}\right\} \mathbf{u}^{j}=\sum_{j=1}^{N E}\left\{\int_{\Gamma_{j}} \mathbf{U} \mathbf{d} \Omega\right\} \mathbf{t}^{j} .
$$

Chamando

$$
\int_{\Gamma} \mathrm{U} \mathbf{d} \Gamma=\mathbf{G}
$$

$\mathrm{e}$

$$
f_{\Gamma_{j}} \mathbf{T} \mathbf{d} \boldsymbol{\Gamma}=\mathbf{H}
$$

tem-se

$$
\sum_{j=1}^{N} H^{l j} u^{j}=\sum_{j=1}^{N} G^{l j} t^{j}
$$

ou, na forma matricial

$$
\mathbf{H u}=\mathbf{G t} .
$$




\section{Capítulo 4}

\section{Teoria de Placas}

Nesta seção, serão apresentadas algumas considerações gerais sobre placas, incluindo as definições e nomenclatura relacionadas. As hipóteses básicas em que se baseiam as teorias de placas serão introduzidas. Uma vez introduzidas, estas hipóteses serão relacionadas com a teoria de Kirchhoff. Com base nesta teoria, serão obtidas as equações constitutivas de placas. Será mostrado o cálculo da rigidez à flexão em direções arbitrárias. Além disso, serão relacionadas as variáveis referentes às três principais condições de contorno: livre, apoiada e engastada. Não obstante, mostrar-se-á a obtenção das equações diferenciais e soluções fundamentais isotrópicas.

\subsection{Considerações gerais}

Na classificação usual da teoria da elasticidade, existem os elementos estruturais unidimensionais (barras, vigas e eixos), os elementos bidimensionais (as lâminas, subclassificadas em chapas, placas e cascas) e os elementos tridimensionais, que são sólidos onde as três dimensões perpendiculares entre si são de ordem de grandeza equivalentes.

As lâminas são elementos estruturais que têm uma das dimensões, a espessura, muito menor em relação às outras duas. A espessura é delimitada ao meio por uma superfície equidistante das faces da lâmina, chamada superfície média. As placas e as cascas são corpos sólidos tridimensionais que apresentam a particularidade de serem sólidos limitados por duas superfícies. As chapas são lâminas com superfície média plana sujeitas a carregamento nesse plano. As placas são lâminas com faces planas e simétricas em relação à superfícies média, plana e, sujeitas a carregamentos aplicados perpendicularmente a esse plano, conforme Figura 4.1. Finalmente, as cascas são lâminas com superfície média curva ou poliédrica.

Uma consideração importante diz respeito à espessura da placa. Uma placa pode ser classificada como espessa, delgada ou fina, correspondendo a cada classificação um 


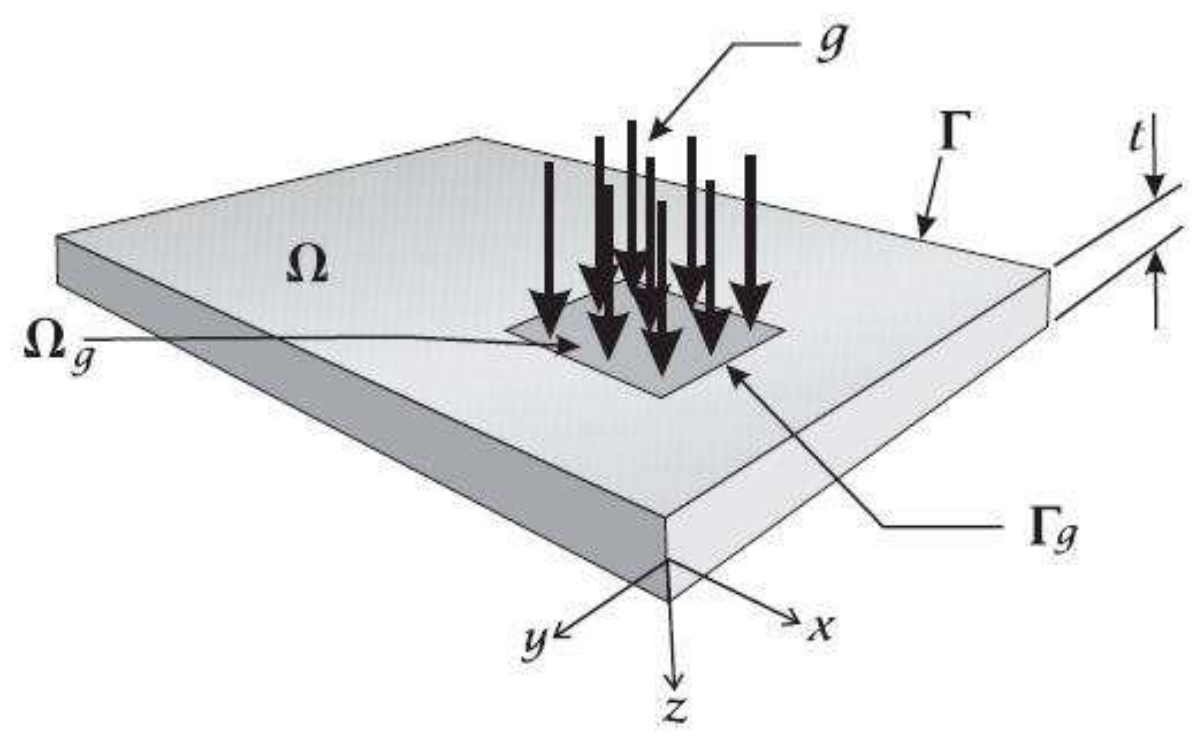

Figura 4.1: Placa fina.

modelo matemático específico. Dependendo da relação $(t / a)$ entre a espessura $(t)$ e a menor dimensão (a) medida no plano médio, a placa pode ser classificada como:

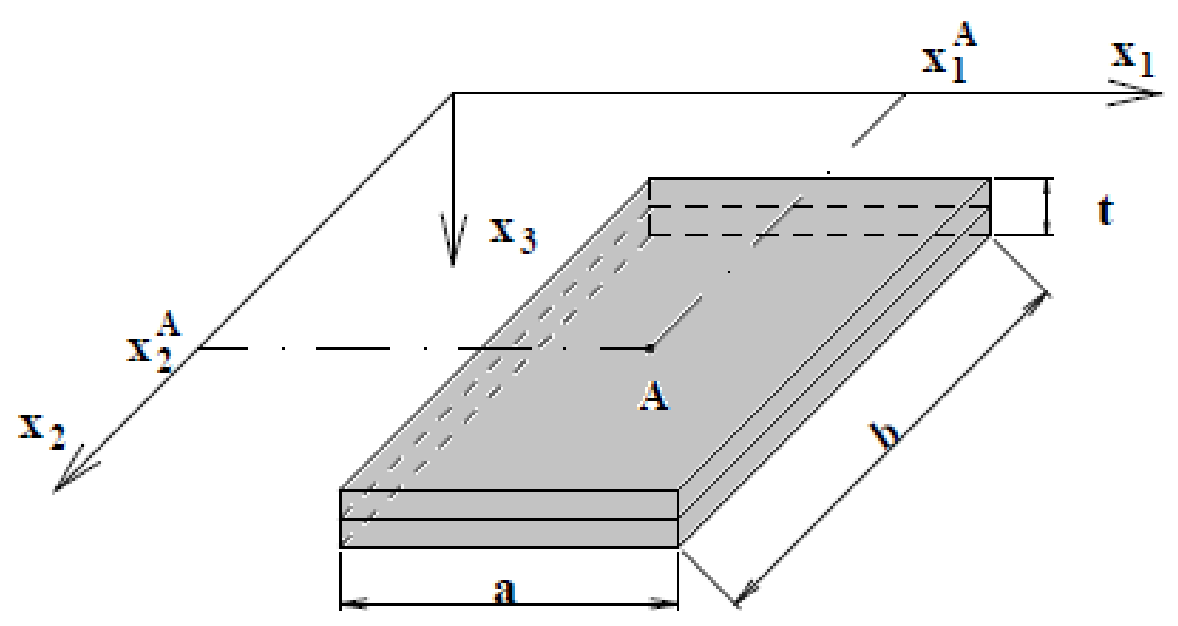

Figura 4.2: Dimensões da placa

- delgada: $t / a<1 / 80$

- fina: $1 / 80 \leq t / a \leq 1 / 5$

- espessa: $t / a>1 / 5$

Em qualquer caso, a espessura pode ser constante ou variável. Estas dimensões, entretanto, foram definidas para materiais metálicos, tais como aço e alumínio. Dependendo das propriedades elásticas do material esta razão entre as dimensões tem 
que ser revista. Uma definição mais completa é baseada no efeito das tensões de cisalhamento normais ao plano da placa. Se o efeito dessas tensões forem desprezíveis no comportamento da estrutura, então, tem-se uma placa fina.

Outra consideração importante a ser feita relaciona-se com a flecha. Flecha é o deslocamento $w$ fora do plano de um dado ponto da superfície média da placa quando esta é submetida a um carregamento, conforme Figura 4.3. A flecha é considerada pequena quando a relação entre o deslocamento $w$ e a espessura $t$ for menor que $1 / 5$.

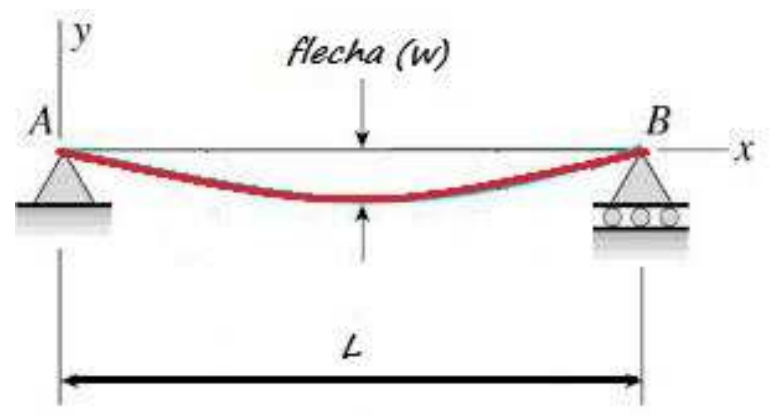

Figura 4.3: Flecha

Sob a ação de um carregamento, a placa se deforma. A superfície média acompanha essa deformação, assumindo uma nova configuração e, recebe o nome de superfície elástica.

\subsection{Hipóteses básicas}

O estudo de placas segue basicamente duas teorias: a teoria de Kirchhoff (1850) e a teoria de Mindlin (1951) ou Reissner (1944).

A formulação clássica de Kirchhoff para placas finas apresenta algumas hipóteses simplificadoras. Basicamente, o uso destas hipóteses tem como aspecto principal a não consideração do efeito dos esforços cortantes nas deformações por flexão. Isto decorre da hipótese de que seções planas e normais ao plano médio permanecem planas e normais após a deformação. Em função das hipóteses apresentadas nesta teoria, assumida como uma teoria de deformações impostas, pode-se deduzir que o deslocamento $w$ depende somente de $x$ e $y$ e que os deslocamentos $u$ e $v$ são lineares em $z$.

Com base nas hipóteses adotadas, a análise do comportamento da placa é feita em regime elástico linear para materiais homogêneos. O equilíbrio é feito na posição não deslocada, utilizando-se a hipótese de pequenas deformações. Sendo assim, se a deflexão da placa é pequena em comparação com sua espessura, quando a mesma está sujeita a um carregamento $g(x ; y)$, as seguintes hipóteses podem ser adotadas: 
- Os deslocamentos normais ao plano da placa são pequenos e as deformações na direção da espessura são pequenas o suficiente para serem desprezadas;

- As tensões normais atuando nos planos paralelos ao plano médio são pequenas, quando comparadas com as outras componentes de tensão, e podem ser desprezadas;

- Os componentes de deslocamento contidas no plano da placa, variam linearmente com a espessura;

- Retas normais ao plano médio na posição não deformada permanecem normais após a deformação. Isto significa desprezar as deformações por cortante que causam distorção;

- Não existem tensões de cisalhamento nas faces externas paralelas ao plano médio da placa.

As teorias de Reissner (tensões impostas) e Mindlin (deslocamentos impostos) para a flexão de placas moderadamente espessas, consideram ainda o efeito da deformação por cortante na formulação do problema. As hipóteses a serem consideradas por estas formulações são semelhantes àquelas apresentadas na teoria de Kirchhoff. Entretanto, estas teorias consideram que as seções normais ao plano médio da placa na posição não deformada, não mais permanecem normais após a deformação. Isto significa incluir as deformações por cortante, que causam distorção na peça.

\subsection{Placas de Kirchhoff}

Uma vez introduzidos todos esses conceitos, pode-se relacioná-los de acordo com a teoria de placas finas, também conhecida como teoria de Kirchhoff, na qual se baseia a formulação desenvolvida nesta tese. São assumidas algumas hipóteses simplificadoras necessárias para se escreverem as equações diferenciais básicas de um elemento de placa fina.

Com relação ao material, assume-se:

- isotrópico, ortotrópico ou anisotrópico;

- homogêneo;

- elástico-linear.

A placa apresenta geometria: 
- fina;

- constante

Quanto ao comportamento da placa em relação ao carregamento, considera-se:

- os deslocamentos transversais são pequenos quando comparados com a espessura da placa (as flechas são pequenas);

- não há deformação do plano médio da placa;

- um plano normal à superfície média da placa, antes da deformação, permanece normal à superfície média deformada, após a deformação;

- as tensões normais e de cisalhamento na direção transversal à placa são desprezíveis.

\subsection{Placas isotrópicas}

Considere uma placa não carregada onde o plano $x y$ coincide com o plano médio, sendo assim, a deflexão em z é igual a zero, Figura 4.4. As componentes do deslocamento num ponto nas direções $x, y$ e $z$ são $u, v$ e $w$, respectivamente. Quando, devido a carregamento laterais, existe deformação, a superfície média num ponto qualquer $\left(x_{a}, y_{a}\right)$ tem deflexão $w$. Os pressupostos fundamentais da teoria de flexão com deflexões pequenas (teoria clássica de placas isotrópicas, homogêneas e finas) baseia-se na geometria das deformações. O deslocamento sofrido por um ponto na direção normal ao plano médio denomina-se deslocamento transversal.
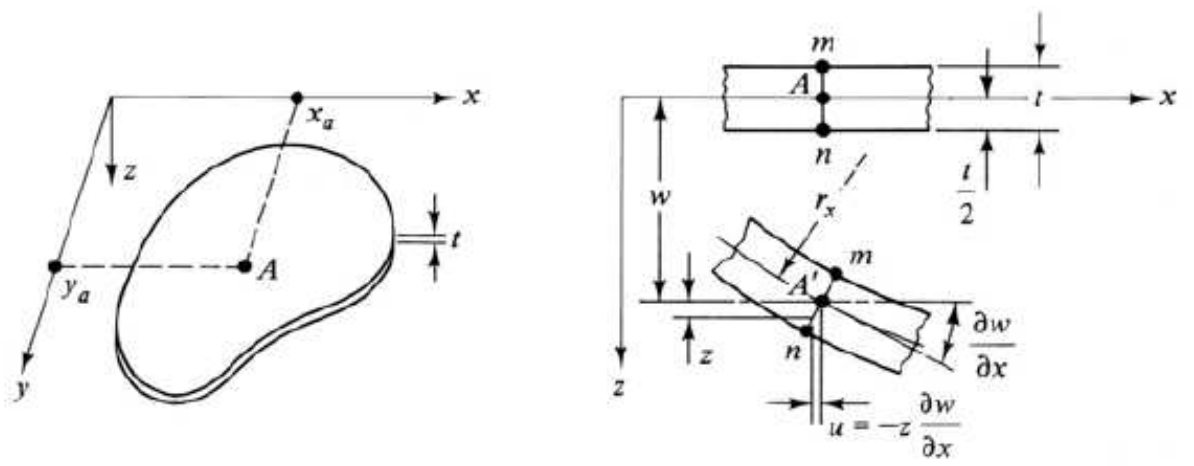

Figura 4.4: Comportamento Geral de Placas. 


\subsubsection{Relações deslocamento-deformação e curvatura}

Como consequência dos pressupostos de Kirchhoff, as relações de extensão-deslocamento observadas na Equações (2.6) e (2.9) resultam:

$$
\epsilon_{x}=\frac{\partial u}{\partial x}, \quad \epsilon_{y}=\frac{\partial v}{\partial y}, \quad \epsilon_{z}=\frac{\partial w}{\partial z}=0
$$

e

$$
\gamma_{x y}=\frac{\partial u}{\partial y}+\frac{\partial v}{\partial x}, \quad \gamma_{x z}=\frac{\partial u}{\partial z}+\frac{\partial w}{\partial x}=0, \quad \gamma_{y z}=\frac{\partial v}{\partial z}+\frac{\partial w}{\partial y}=0
$$

onde

$$
\gamma_{x y}=\gamma_{y x}, \quad \gamma_{x z}=\gamma_{z x}, \quad \gamma_{y z}=\gamma_{z y}
$$

Integrando-se $\varepsilon_{z}$ da Equação (4.1), tem-se:

$$
w=w(x, y)
$$

indicando que a deflexão lateral não varia na espessura da placa.

Da mesma forma, integrando as expressões de $\gamma_{x z}$ e $\gamma_{y z}$, tem-se:

$$
u=-z \frac{\partial w}{\partial x}+u_{0}(x, y) ; \quad v=-z \frac{\partial w}{\partial y}+v_{0}(x, y)
$$

Torna-se claro que $u_{0}(x, y)$ e $v_{0}(x, y)$ representam, respectivamente, os valores de $u$ e de $v$ na superfície média. Conclui-se que $u_{0}=v_{0}=0$. Assim,

$$
u=-z \frac{\partial w}{\partial x} ; \quad v=-z \frac{\partial w}{\partial y}
$$

Substituindo as Equações (4.6) nas Equações (4.1) e (4.2) de extensões, obtém-se:

$$
\varepsilon_{x}=-z \frac{\partial^{2} w}{\partial x^{2}} ; \quad \varepsilon_{y}=-z \frac{\partial^{2} w}{\partial y^{2}} ; \quad \gamma_{x y}=-2 z \frac{\partial^{2} w}{\partial x \partial y}
$$

A curvatura plana é definida como a taxa de variação do ângulo de declive da curva em relação à distância ao longo da curva. As derivadas parciais das Equações (4.7) representam as curvaturas da placa. Assim, as curvaturas $\kappa$ na superfície média em planos paralelos ao plano $x z, y z$ e $x y$ são, respectivamente

$$
\begin{gathered}
\frac{1}{r_{x}}=\frac{\partial}{\partial x}\left(\frac{\partial w}{\partial x}\right)=\kappa_{x}, \\
\frac{1}{r_{y}}=\frac{\partial}{\partial y}\left(\frac{\partial w}{\partial y}\right)=\kappa_{y}, \\
\frac{1}{r_{x y}}=\frac{\partial}{\partial x}\left(\frac{\partial w}{\partial y}\right)=\kappa_{x y}
\end{gathered}
$$


onde $\kappa_{x y}=\kappa_{y x}$.

A última expressão também é conhecida como a torção do plano médio em relação $\operatorname{aos}$ eixos $x$ e $y$.

Assim, as relações extensão-curvatura da placa podem representar-se na seguinte forma:

$$
\varepsilon_{x}=-z \kappa_{x}, \quad \varepsilon_{y}=-z \kappa_{y}, \quad \gamma_{x y}=-2 z \kappa_{x y} .
$$

\subsubsection{Tensões e Resultantes de Tensões}

No caso de um estado de tensão tridimensional, as tensões e as deformações estão relacionadas pela lei de Hooke generalizada, válida para um material isotrópico homogêneo como observado nas Equações (2.12) e (2.13). Substituindo $\varepsilon_{z}=\gamma_{y z}=\gamma_{x z}=0$, obtém-se as relações tensão-extensão da placa fina:

$$
\sigma_{x}=\frac{E}{1-\nu^{2}}\left(\varepsilon_{x}-\nu \varepsilon_{y}\right), \quad \sigma_{y}=\frac{E}{1-\nu^{2}}\left(\varepsilon_{y}-\nu \varepsilon_{x}\right), \quad \tau_{x y}=G \gamma_{x y} .
$$

onde $\nu$ é o coeficiente de Poisson.

Introduzindo as curvaturas da placa, as Equações (4.10) ficam com a forma seguinte:

$$
\begin{aligned}
\sigma_{x}=-\frac{E z}{1-\nu^{2}}\left(\kappa_{x}+\nu \kappa_{y}\right) & =-\frac{E z}{1-\nu^{2}}\left(\frac{\partial^{2} w}{\partial x^{2}}+\nu \frac{\partial^{2} w}{\partial y^{2}}\right), \\
\sigma_{y}=-\frac{E z}{1-\nu^{2}}\left(\kappa_{y}+\nu \kappa_{x}\right) & =-\frac{E z}{1-\nu^{2}}\left(\frac{\partial^{2} w}{\partial y^{2}}+\nu \frac{\partial^{2} w}{\partial x^{2}}\right), \\
\tau_{x y} & =-\frac{E z}{1-\nu} \kappa_{x y}=-\frac{E z}{1-\nu} \frac{\partial^{2} w}{\partial x \partial y} .
\end{aligned}
$$

Pode-se observar que a tensão desaparece na superfície média e varia linearmente ao longo da espessura da placa.

As tensões distribuídas pela espessura da placa produzem momentos fletores, momentos torsores e forças de corte verticais, Figura 4.5. Estes momentos e forças por unidade de comprimento são conhecidas por resultantes de tensões.

Da Figura 4.5, para a tensão $\sigma_{x}$, tem-se:

$$
\int_{-t / 2}^{t / 2} z \sigma_{x} d y d z=d y \int_{-t / 2}^{t / 2} z \sigma_{x} d z=M_{x} d y
$$

Da mesma forma, para as outras tensões obtêm-se as seguintes resultantes de tensão:

$$
\left\{\begin{array}{l}
M_{x} \\
M_{y} \\
M_{x y}
\end{array}\right\}=\int_{-t / 2}^{t / 2}\left\{\begin{array}{c}
\sigma_{x} \\
\sigma_{y} \\
\tau_{x y}
\end{array}\right\} z d z
$$




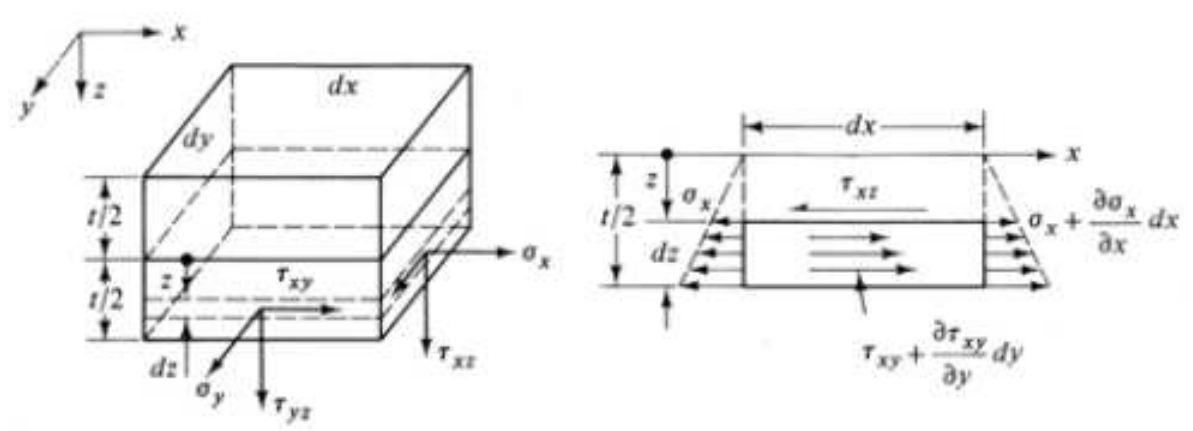

Figura 4.5: Resultantes de tensões.

onde $M_{x y}=M_{y x}$.

Para as forças cortantes por unidade de comprimento, tem-se:

$$
\left\{\begin{array}{l}
Q_{x} \\
Q_{y}
\end{array}\right\}=\int_{-t / 2}^{t / 2}\left\{\begin{array}{l}
\tau_{x z} \\
\tau_{y z}
\end{array}\right\} z d z
$$

É importante notar que apesar da teoria de placas finas omitir o efeito das deformações $\gamma_{x z}=\tau_{x z} / G$ e $\gamma_{y z}=\tau_{y z} / G$ na flexão, as forças verticais $Q_{x}$ e $Q_{y}$ não são desprezáveis.

Substituindo as equações das tensões em função dos deslocamentos nas equações dos momentos, pode-se deduzir as fórmulas dos momentos fletores e torsores em função das curvaturas e deflexões.

$$
\begin{gathered}
M_{x}=-D\left(\kappa_{x}+\nu \kappa_{y}\right)=-D\left(\frac{\partial^{2} w}{\partial x^{2}}+\nu \frac{\partial^{2} w}{\partial y^{2}}\right), \\
M_{y}=-D\left(\kappa_{y}+\nu \kappa_{x}\right)=-D\left(\frac{\partial^{2} w}{\partial y^{2}}+\nu \frac{\partial^{2} w}{\partial x^{2}}\right) \\
M_{x y}=-D(1-\nu) \kappa_{x y}=-D(1-\nu) \frac{\partial^{2} w}{\partial x \partial y} .
\end{gathered}
$$

onde $D$ é a rigidez de flexão dada por

$$
D=\frac{E t^{3}}{12\left(1-\nu^{2}\right)}
$$

Substituindo as equações dos momentos nas equações das tensões, pode-se obter as tensões em função dos momentos.

$$
\sigma_{x}=\frac{12 M_{x} z}{t^{3}}, \quad \sigma_{y}=\frac{12 M_{y} z}{t^{3}}, \quad \tau_{x y}=\frac{12 M_{x y} z}{t^{3}} .
$$

A tensão máxima ocorre nas superfícies superior e inferior $(z= \pm t / 2)$ da placa. Desta análise pode observa-se que existe uma correspondência direta entre os momentos e as tensões. Conclui-se que as equações de transformação das tensões e dos momentos são análogas. 
A análise do círculo de Mohr e todas as conclusões sobre as tensões podem ser aplicadas aos momentos. A determinação das tensões $\sigma_{z}, \tau_{x z}$ e $\tau_{y z}$ através da lei de Hooke não é possível porque não se relacionam com as extensões.

Através das Equações diferenciais de equilíbrio (2.15) as demais componentes de tensões podem ser calculadas da seguinte forma:

$$
\begin{aligned}
& \frac{\partial \sigma_{x}}{\partial x}+\frac{\partial \tau_{x y}}{\partial y}+\frac{\partial \tau_{x z}}{\partial z}=0 \\
& \frac{\partial \sigma_{y}}{\partial y}+\frac{\partial \tau_{y x}}{\partial x}+\frac{\partial \tau_{y z}}{\partial z}=0 \\
& \frac{\partial \sigma_{z}}{\partial z}+\frac{\partial \tau_{z x}}{\partial x}+\frac{\partial \tau_{z y}}{\partial y}=0
\end{aligned}
$$

As tensões de cisalhamento $\tau_{x z}$ e $\tau_{y z}$ das Equações (4.18) depois de integrar, são dadas por:

$$
\begin{gathered}
\tau_{x z}=\int_{z}^{t / 2}\left(\frac{\partial \sigma_{x}}{\partial x}+\frac{\partial \tau_{x y}}{\partial y}\right) d z=-\frac{E}{2\left(1-\nu^{2}\right)}\left(\frac{t^{2}}{4}-z^{2}\right)\left[\frac{\partial}{\partial x}\left(\frac{\partial^{2} w}{\partial x^{2}}+\frac{\partial^{2} w}{\partial y^{2}}\right)\right] \\
\tau_{y z}=\int_{z}^{t / 2}\left(\frac{\partial \sigma_{x}}{\partial y}+\frac{\partial \tau_{x y}}{\partial x}\right) d z=-\frac{E}{2\left(1-\nu^{2}\right)}\left(\frac{t^{2}}{4}-z^{2}\right)\left[\frac{\partial}{\partial y}\left(\frac{\partial^{2} w}{\partial x^{2}}+\frac{\partial^{2} w}{\partial y^{2}}\right)\right] .
\end{gathered}
$$

Pode-se observar que as distribuições de $\tau_{x z}$ e $\tau_{y z}$ na espessura da placa variam de acordo com uma lei parabólica.

A componente $\sigma_{z}$ é obtida usando a terceira equação de equilíbrio, substituindo para $\tau_{x z}$ e $\tau_{y z}$ e integrando.

$$
\sigma_{z}=-\frac{E}{2\left(1-\nu^{2}\right)}\left(\frac{t^{3}}{12}-\frac{t^{2} z}{4}+\frac{z^{3}}{3}\right)\left[\left(\frac{\partial^{2}}{\partial x^{2}}+\frac{\partial^{2}}{\partial y^{2}}\right)\left(\frac{\partial^{2} w}{\partial x^{2}}+\frac{\partial^{2} w}{\partial y^{2}}\right)\right]
$$

A tensão normal $\sigma_{z}$ varia na forma de uma parábola cúbica ao longo da espessura da placa. As tensões cisalhante na direção $z$ são consideradas muito pequenas quando comparadas com as outras tensões.

\subsection{Equações constitutivas}

As componentes da tensão (resultantes de tensão) variam, geralmente, de ponto para ponto numa placa carregada. Essas variações são governadas pelas condições de equilíbrio da estática. O cumprimento destas condições estabelece certas relações conhecidas por equações de equilíbrio. 


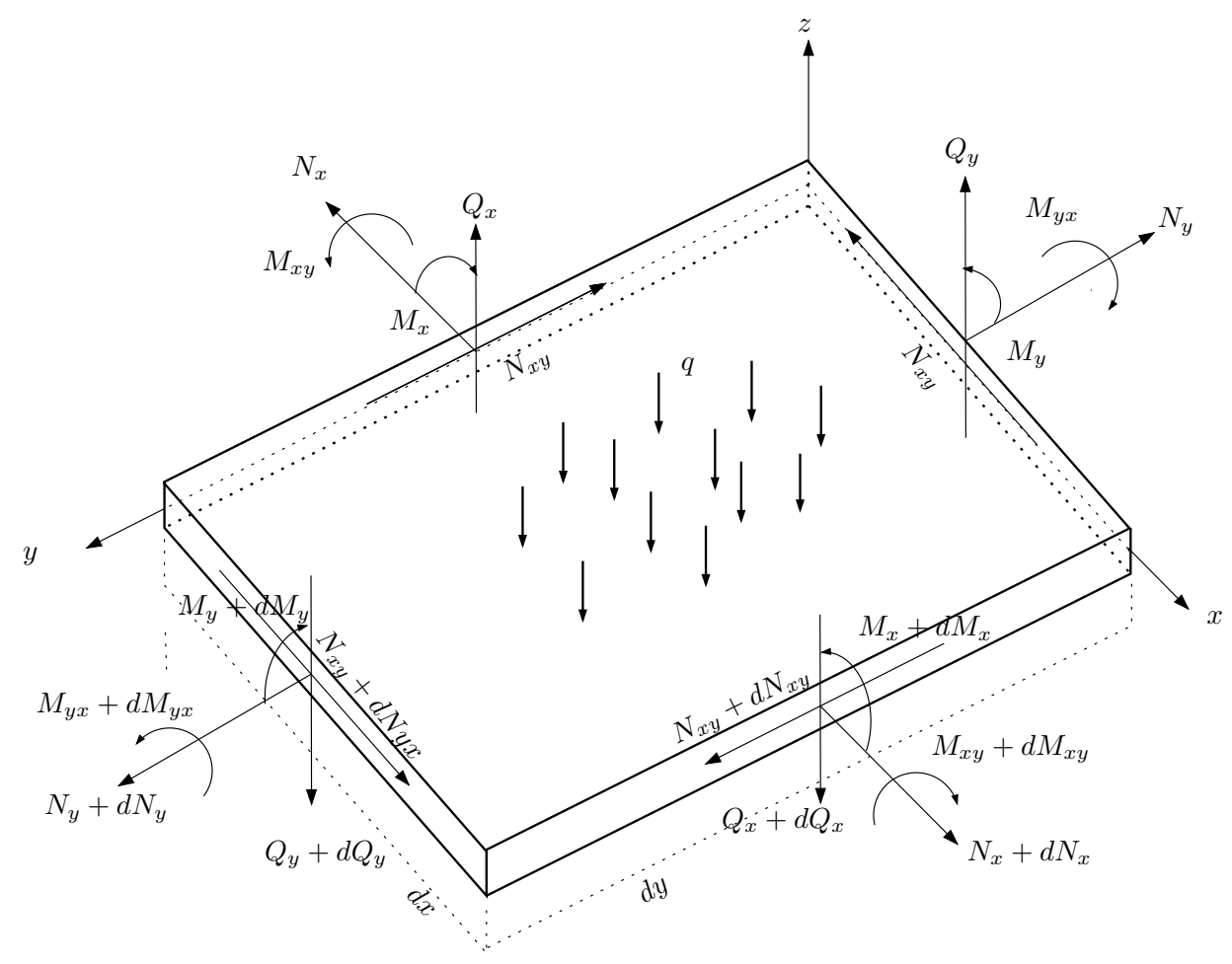

Figura 4.6: Equilíbrio de forças e momentos em uma placa

A Figura 4.6 mostra um elemento de dimensões $d x$ e $d y$ extraído de uma placa submetida a uma carga $q=q(x ; y)$. Nessa figura indicam-se os esforços internos por unidade de comprimento segundo os sentidos considerados positivos.

Assume-se que a inclusão do peso da placa, sendo um valor pequeno, no carregamento $q$ não afeta a precisão do resultado. Além disso, assume-se que as componentes de força e de momento estão distribuídas uniformemente em cada uma das faces. Na Figura 4.6, elas estão representadas por um vetor único de valores médios, aplicado no centro de cada face. Com uma mudança de posição, por exemplo, da face esquerda para a face direita, a componente do momento $M_{x}$ que atua na face negativa de $x$ varia em valor relativamente à face positiva de $x$. Esta variação pode ser representada por uma série de Taylor truncada.

$$
M_{x}+\frac{\partial M_{x}}{\partial x} d x
$$

Usa-se a derivada parcial, pois $M_{x}$ é função de $x$ e $y$. Tratando todas as componentes de forma similar, obtém-se o estado das resultantes de tensão a partir da Figura 4.6.

O equilíbrio de forças na direção $w$ nesse elemento é dado por

1. O somatório de forças no eixo $z$

$$
\frac{\partial Q_{x}}{\partial x} d x d y+\frac{\partial Q_{y}}{\partial y} d x d y+q d x d y=0
$$


a partir do qual

$$
\frac{\partial Q_{x}}{\partial x}+\frac{\partial Q_{y}}{\partial y}+q=0 .
$$

2. O somatório de momentos sobre o eixo $x$

$$
\frac{\partial M_{x y}}{\partial x} d x d y+\frac{\partial M_{y}}{\partial y} d x d y-Q_{y} d x d y=0,
$$

$\mathrm{Ou}$

$$
\frac{\partial M_{x y}}{\partial x}+\frac{\partial M_{y}}{\partial y}-Q_{y}=0,
$$

3. O somatório de momentos sobre o eixo y

$$
\frac{\partial M_{y x}}{\partial y}+\frac{\partial M_{x}}{\partial x}-Q_{x}=0 .
$$

As seguintes expressões, equações (4.25) e (4.26), que são as forças de cisalhamento $Q_{x}$ and $Q_{y}$ podem sem expressas em termos dos momentos:

$$
\begin{aligned}
Q_{x} & =\frac{\partial M_{x}}{\partial x}+\frac{\partial M_{x y}}{\partial y}, \\
Q_{y} & =\frac{\partial M_{x y}}{\partial x}+\frac{\partial M_{y}}{\partial y} .
\end{aligned}
$$

\subsubsection{Equação diferencial}

Conforme mostrado por Fernandes (1974), as derivadas das Equações (4.15) são dadas por:

$$
\begin{aligned}
\frac{\partial M_{x}}{\partial x} & =-D\left(\frac{\partial^{3} w}{\partial x^{3}}+\nu \frac{\partial^{3} w}{\partial x \partial y^{2}}\right), \\
\frac{\partial M_{y}}{\partial y} & =-D\left(\frac{\partial^{3} w}{\partial y^{3}}+\nu \frac{\partial^{3} w}{\partial x^{2} \partial y}\right), \\
\frac{\partial M_{x y}}{\partial x} & =D(1-\nu)\left(\frac{\partial^{3} w}{\partial x^{2} \partial y}\right), \\
\frac{\partial M_{x y}}{\partial y} & =D(1-\nu)\left(\frac{\partial^{3} w}{\partial x \partial y^{2}}\right) .
\end{aligned}
$$

Substituindo as expressões, Equações (4.28) a (4.31), nas Equações (4.25) a (4.26), obtém-se $Q_{x}$ e $Q_{y}$ em função do deslocamento $w$, 


$$
\begin{aligned}
& Q_{x}=-D\left(\frac{\partial^{3} w}{\partial x^{3}}+\frac{\partial^{3} w}{\partial x \partial y^{2}}\right) \\
& Q_{y}=-D\left(\frac{\partial^{3} w}{\partial y^{3}}+\frac{\partial^{3} w}{\partial x^{2} \partial y}\right)
\end{aligned}
$$

Considerando-se a simetria de momentos $M_{x y}=M_{y x}$. Substituindo as Equações (4.32) nas Equações (4.23), encontra-se o seguinte:

$$
\frac{\partial^{2} M_{x}}{\partial x^{2}}+2 \frac{\partial^{2} M_{x y}}{\partial x \partial y}+\frac{\partial^{2} M_{y}}{\partial y^{2}}=-q(x, y)
$$

Finalmente, introduzindo-se as expressões de $M_{x}, M_{y}$ e $M_{x y}$ das Equações (4.15) na Equação (4.33), obtém-se

$$
\frac{\partial^{4} w}{\partial x^{4}}+2 \frac{\partial^{4} w}{\partial x^{2} \partial y^{2}}+\frac{\partial^{4} w}{\partial y^{4}}=\frac{g}{D}
$$

Esta é a equação diferencial de deslocamento para análise de flexão de placas finas baseado na Teoria de Kirchhoff. Essa equação foi obtida por Lagrange in 1811. Matematicamente, a equação diferencial (4.34) pode ser classificada como uma equação diferencial linear parcial de quarta ordem com coeficientes constantes.

A equação (4.34) pode ser reescrita, obtendo-se:

$$
\nabla^{2}\left(\nabla^{2} w\right)=\nabla^{4} w=\frac{q}{D}
$$

onde

$$
\nabla^{4}()=\frac{\partial^{4}}{\partial x^{4}}+2 \frac{\partial^{4}}{\partial x^{2} \partial y^{2}}+\frac{\partial^{4}}{\partial y^{4}}
$$

é comumente chamado de operador biharmônico.

\subsection{Cálculo da rigidez à flexão em direções arbitrárias}

Ao aplicarem-se momentos $M_{x}$ e $M_{y}$ em planos perpendiculares entre si, surgem momentos de flexão e de torção em dois planos arbitrários e perpendiculares entre si. Tomando um elemento $A B C D$ de uma placa solicitada nas bordas por momentos $M_{x}$ e $M_{y}$, observa-se que, as faces desse elemento estão submetidas a $M_{x}$ e $M_{y}$, Figura 4.7.

Como mostrado na figura 4.8, tomando-se os eixos $n$ e $t$ perpendiculares entre si e, formando o ângulo $\alpha$ com os eixos $x$ e $y$, respectivamente, os momentos provocados por $M_{x}$ e $M_{y}$ em planos paralelos a essas direções serão $M_{n}$ e $M_{n t}$. 

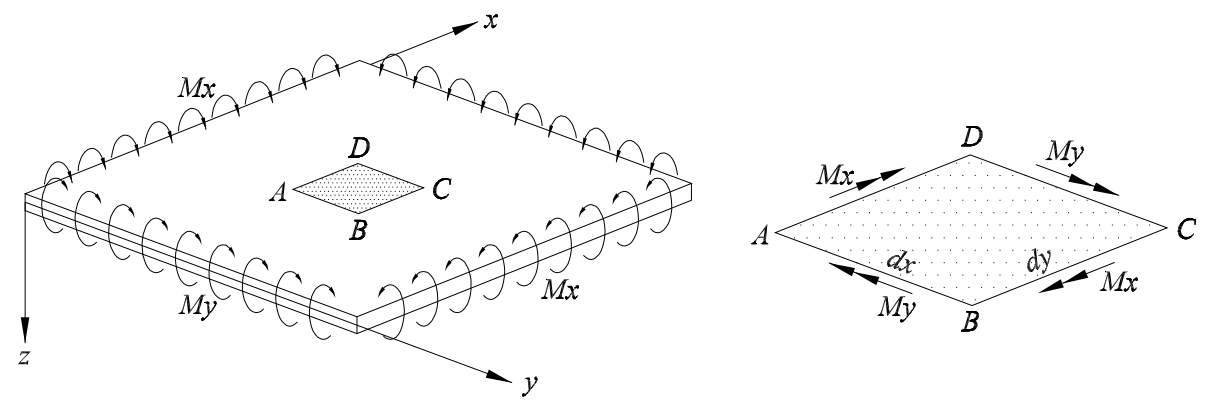

Figura 4.7: Elemento de placa submetido a momentos nas bordas.

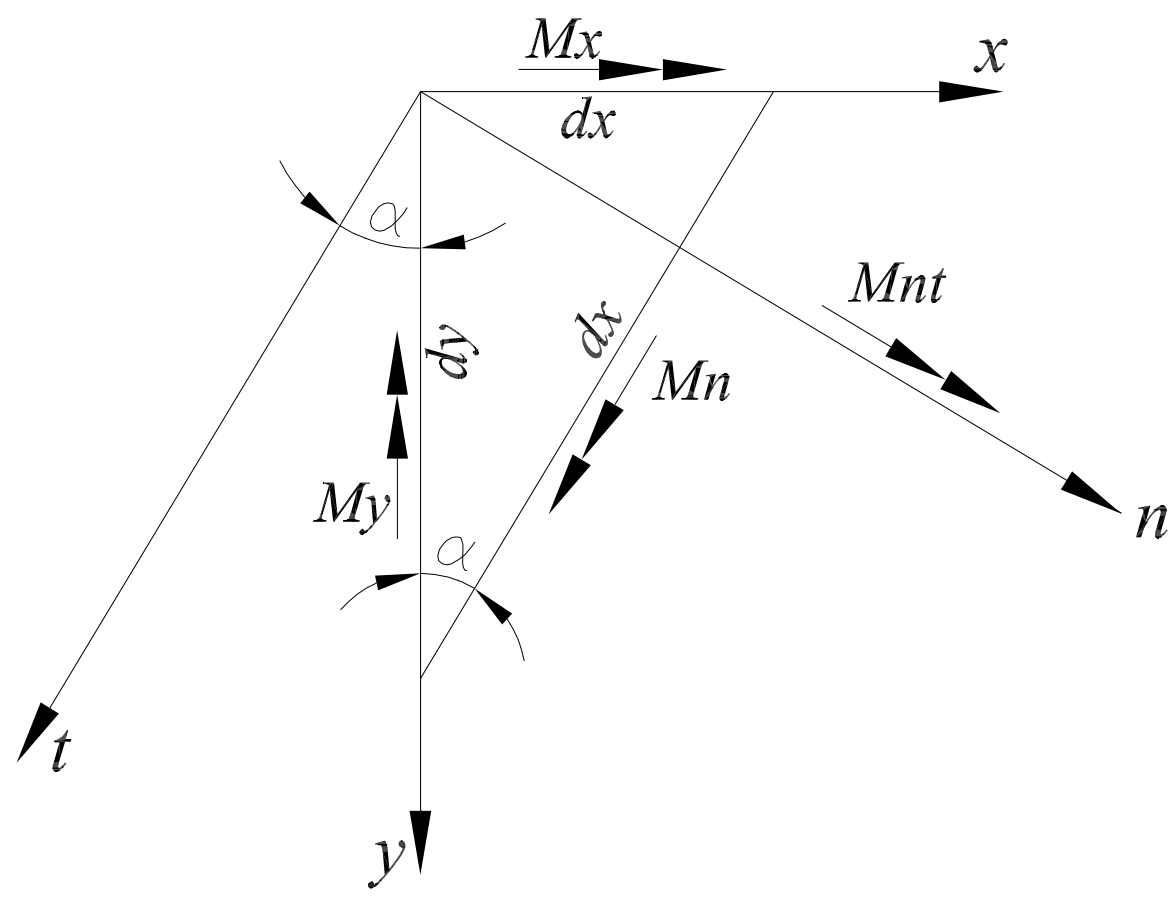

Figura 4.8: Elemento de placa submetido a momentos em direções arbitrárias.

O momento fletor $M_{n}$ e o momento volvente $M_{n t}$ são considerados positivos pela regra da mão direita de acordo com os eixo $n$ e $t$.

Fazendo-se o equilíbrio de momentos em torno do eixo $t$ tem-se $M_{n}$,

$$
M_{n}=M_{x} \cos ^{2} \alpha+M_{y} \operatorname{sen}^{2} \alpha+2 M_{x y} \operatorname{sen} \alpha \cos \alpha
$$

e, fazendo-se o equilíbrio em torno do eixo $n$ tem-se $M_{n t}$

$$
M_{n t}=\left(M_{y}-M_{x}\right) \operatorname{sen} \alpha \cos \alpha+M_{x y}\left(\cos ^{2} \alpha-\operatorname{sen}^{2} \alpha\right)
$$

Da mesma forma, a força cortante $Q_{n}$ pode ser escrita como:

$$
Q_{n}=Q_{x} \cos \alpha+Q_{y} \operatorname{sen} \alpha
$$


Kirchhoff (1850), mostrou que as condições de contorno de força cortante $Q_{n}$ e momento volvente $M_{n t}$, Figura 4.9, podem ser escritas como uma única condição de contorno dada por

$$
V_{n}=Q_{n}+\frac{\partial M_{n t}}{\partial t}
$$

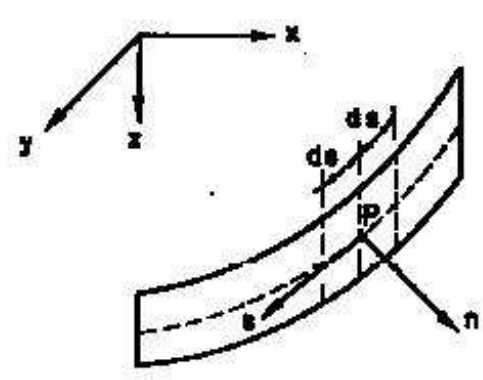

a)

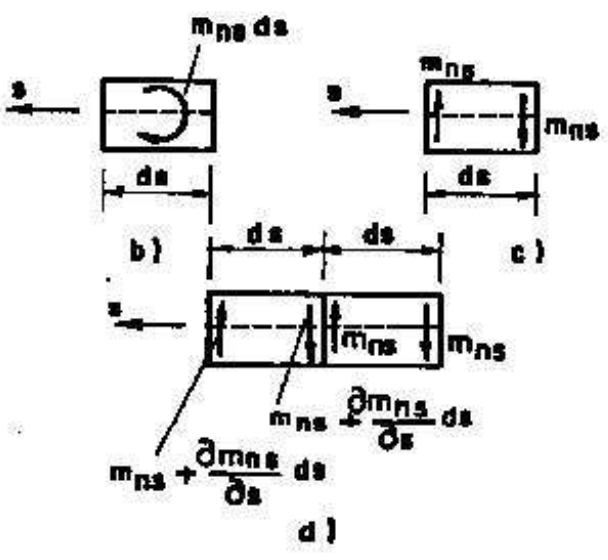

Figura 4.9: Momento volvente no contorno. Paiva (1987)

Portanto, as condições de contorno de deslocamentos são a deflexão $w$ e a rotação $\frac{\partial w}{\partial n}$ e as condições de contorno de carregamento são a força cortante equivalente $V_{n}$ e o momento fletor $M_{n}$.

\subsection{Condições de contorno}

Para solucionar o problema de placas, é necessária a prescrição das condições de contorno do problema em questão. Considerando-se o sistema de coordenadas genérico $n t$, conforme Figura 4.9, as condições de contorno podem ser estabelecidas segundo a Tabela 4.1 abaixo:

Tabela 4.1: Condições de contorno.

\begin{tabular}{ccccc}
\hline Condições de contorno & \multicolumn{2}{c}{ Conhecidas } & Desconhecidas \\
\hline Engastada & $w=0$ & $\frac{\partial w}{\partial n}=0$ & $V_{n}$ & $M_{n}$ \\
Apoiada & $w=0$ & $M_{n}=0$ & $\frac{\partial w}{\partial n}$ & $V_{n}$ \\
Livre & $V_{n}=0$ & $M_{n}=0$ & $w$ & $\frac{\partial w}{\partial n}$ \\
\hline
\end{tabular}

Com relação às condições de contorno, a teoria de Kirchhoff determina que apenas duas condições são suficientes para a completa determinação de $w$. 


\subsection{Soluções fundamentais isotrópicas}

Na formulação das equações integrais do método dos elementos de contorno, por meio do teorema da reciprocidade, dois estados de tensão num corpo são relacionados. Um estado de tensão, é conhecido e, o outro necessita ser determinado. O estado a ser determinado está relacionado com o problema a ser resolvido, ou seja, com a análise a ser realizada. O problema a ser resolvido pode ser representado por um meio, um corpo ou uma peça mecânica, o qual tem geometria, carregamentos e condições de contorno definidos. O estado conhecido é dado pela resposta de um corpo de domínio infinito, cujas propriedades do material são as mesmas do estado a ser determinado à aplicação de uma carga concentrada unitária e pontual. À esta resposta, é dado o nome de solução fundamental.

A formulação do método dos elementos de contorno requer o conhecimento da solução fundamental. No caso particular de placas, a solução fundamental é dada pelo deslocamento $w$ em um ponto $P(x, y)$ qualquer do domínio, chamado de ponto campo, devido a aplicação de uma carga unitária $q$ em um ponto $Q\left(x_{0}, y_{0}\right)$ qualquer, chamado ponto fonte conforme Figura 3.1.

A solução fundamental do deslocamento transversal de placas fletidas é calculado fazendo o termo não-homogêneo da equação diferencial (4.34) igual a uma força concentrada dada por uma função delta de Dirac $\delta(Q, P)$. Esta solução fundamental é dada por:

$$
w^{*}=\frac{1}{8 \pi D_{22}} r^{2}(\log r)
$$

onde $r^{2}=\left[\left(x-x_{0}\right)^{2}+\left(y-y_{0}\right)^{2}\right]$ é a distância do ponto fonte ao ponto campo. Essa escolha, deve-se ao método dos elementos de contorno exigir a utilização de diversas derivadas da solução fundamental $w$. As demais soluções fundamentais, derivadas desta conforme Paiva (1987), são dadas por: 


$$
\begin{aligned}
V_{n}^{*}= & \frac{1}{4 \pi \rho}(\mathbf{n} \cdot \mathbf{r})\left[-3+\nu+2(1-\nu)(\mathbf{s} \cdot \mathbf{r})^{2}\right] \\
m_{n}^{*}= & -\frac{1}{8 \pi}\left[2(\mathbf{n} \cdot \mathbf{r})^{2}(1-\nu)+2 \nu+(1+\nu)(1+2 \log r)\right] \\
\frac{\partial w^{*}}{\partial n}= & \frac{1}{8 \pi D_{22}}[(\mathbf{n} \cdot \mathbf{r}) r(1+2 \log r)] \\
\frac{\partial w^{*}}{\partial m}= & -\frac{1}{8 \pi D_{22}}[(\mathbf{n} \cdot \mathbf{r}) r(1+2 \log r] \\
\frac{\partial v_{n}^{*}}{\partial m}= & -\frac{1}{4 \pi r^{2}}[(-\mathbf{m} \cdot \mathbf{n}+2(\mathbf{n} \cdot \mathbf{r})(\mathbf{n} \cdot \mathbf{r}))(3-\nu)+ \\
\frac{\partial m_{n}^{*}}{\partial m}= & \left.\left.\frac{1}{4 \pi r}[-2(\mathbf{n} \cdot \mathbf{r})(-\mathbf{m} \cdot \mathbf{\text { m }})(2 \mathbf{m} \cdot \mathbf{s}(\mathbf{n} \cdot \mathbf{r})+(\mathbf{m} \cdot \mathbf{n} \cdot \mathbf{n})(\mathbf{n} \cdot \mathbf{r}))(1-\nu)+\mathbf{r})-\mathbf{n} \cdot \mathbf{r} \cdot \mathbf{r}\right)(1+\nu)\right] \\
\frac{\partial^{2} w^{*}}{\partial n \partial m}= & \left.-\frac{1}{4 \pi D_{22}}[\mathbf{n} \cdot \mathbf{r}(\mathbf{s} \cdot \mathbf{r}))\right]
\end{aligned}
$$

onde $\mathbf{n}$ é o vetor unitário normal ao contorno no ponto campo, $\mathbf{m}$ é o vetor unitário normal ao contorno no ponto fonte, s é o vetor unitário tangente ao contorno no ponto fonte e $\mathbf{r}$ é o vetor unitário na direção da reta que passa pelo ponto fonte e ponto campo. 


\section{Capítulo 5}

\section{Equações do Método dos Elementos de Contorno para Placas de Kirchhoff}

Neste capítulo, será apresentada de forma detalhada a formulação para obtenção da equação integral de contorno e a solução do sistema de equações para os $N$ números de elementos.

\subsection{Formulação integral}

Para a determinação da equação integral para pontos do domínio da placa, seja uma placa de domínio finito $\Omega$ e contorno $\Gamma$ contida em outra de domínio infinito $\Omega_{\infty}$ e contorno $\Gamma_{\infty}$. Conforme a Figura 5.1, a placa finita está submetida a um carregamento $q$ distribuído em uma área $\Omega_{q}$, Paiva (1987).
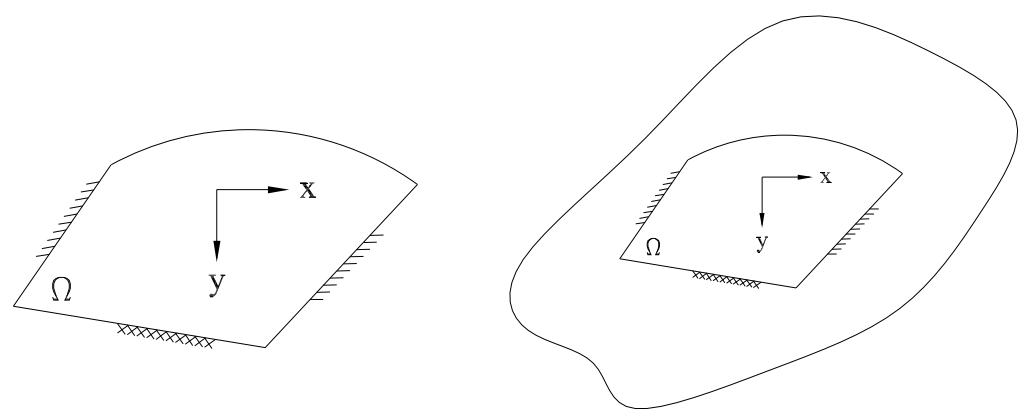

Figura 5.1: Placa finita contida em uma placa infinita.

O teorema de Betti, Kane (1993), é obtido considerando-se que a placa de domínio finito é submetida a dois carregamentos não simultâneos $q$ e $q^{*}$, associados a superfícies elásticas $w$ e $w^{*}$, respectivamente. São identificados dois estados de tensão $\sigma$ e $\sigma^{*}$, com 
seus respectivos estados de deformação $\varepsilon$ e $\varepsilon^{*}$. Podemos relacionar dois estados de tensão-deformação de um material linear como:

$$
\int_{\Omega} \sigma_{i j}^{*} \varepsilon_{i j} d \Omega=\int_{\Omega} \sigma_{i j} \varepsilon_{i j}^{*} d \Omega
$$

Escrevendo o lado direito da Equação (5.1) na notação de von Karman, temos:

$$
\int_{\Omega} \sigma_{i j} \varepsilon_{i j}^{*} d \Omega=\int_{\Omega}\left(\sigma_{x} \varepsilon_{x}^{*}+\sigma_{y} \varepsilon_{y}^{*}+\sigma_{z} \varepsilon_{z}^{*}+\tau_{x y} \gamma_{x y}^{*}+\tau_{x z} \gamma_{x z}^{*}+\tau_{y z} \gamma_{y z}^{*}\right) d \Omega
$$

Desconsiderando as tensões normais à superfície média da placa, a Equação (5.2) é escrita como:

$$
\int_{\Omega} \sigma_{i j} \varepsilon_{i j}^{*} d \Omega=\int_{\Omega}\left(\sigma_{x} \varepsilon_{x}^{*}+\sigma_{y} \varepsilon_{y}^{*}+\tau_{x y} \gamma_{x y}^{*}\right) d \Omega .
$$

Substituindo as Equações (2.21) e (2.23) na Equação (5.3), pode-se escrever o primeiro termo da integral do lado direito da Equação (5.3) como:

$$
\int_{\Omega} \sigma_{x} \varepsilon_{x}^{*} d \Omega=\int_{\Omega}\left[\int_{z}\left(B_{11} \frac{\partial^{2} w}{\partial x^{2}}+B_{12} \frac{\partial^{2} w}{\partial y^{2}}+2 B_{16} \frac{\partial^{2} w}{\partial x \partial y}\right)\left(z \frac{\partial^{2} w}{\partial x^{2}}\right) d z\right] d \Omega .
$$

Integrando (5.4) ao longo da espessura da placa, tem-se:

$$
\int_{\Omega} \sigma_{x} \varepsilon_{x}^{*} d \Omega=\int_{\Omega}\left(D_{11} \frac{\partial^{2} w}{\partial x^{2}}+D_{12} \frac{\partial^{2} w}{\partial y^{2}}+2 D_{16} \frac{\partial^{2} w}{\partial x \partial y}\right) \frac{\partial^{2} w}{\partial x^{2}} d \Omega=\int_{\Omega} m_{x} \frac{\partial^{2} w}{\partial x^{2}} d \Omega .
$$

Para obter as equações do método dos elementos de contorno, é necessário transformar as integrais de domínio em integrais de contorno.

Considere duas funções $f(x)$ e $g(x)$. A derivada de seu produto pode ser escrita como:

$$
\frac{\partial}{\partial x}[f(x) g(x)]=\frac{\partial f(x)}{\partial x} g(x)+\frac{\partial g(x)}{\partial x} f(x) .
$$

Usando a propriedade de derivação (5.6) na Equação (5.5), pode-se escrever:

$$
\int_{\Omega} \sigma_{x} \varepsilon_{x}^{*} d \Omega=-\int_{\Omega}\left[\frac{\partial}{\partial x}\left(m_{x} \frac{\partial w^{*}}{\partial x}\right)-\frac{\partial w^{*}}{\partial x} \frac{\partial m_{x}}{\partial x}\right] d \Omega .
$$

Usando o teorema de Green (Kane, 1994), a Equação (5.7) pode ser escrita como:

$$
\int_{\Omega} \sigma_{x} \varepsilon_{x}^{*} d \Omega=-\int_{\Gamma} m_{x} \frac{\partial w^{*}}{\partial x} \cos \alpha d \Gamma+\int_{\Omega} \frac{\partial w^{*}}{\partial x} \frac{\partial m_{x}}{\partial x} d \Omega .
$$

Aplicando a propriedade de derivação (5.6) no segundo termo do lado direito da Equação (5.8), tem-se: 


$$
\int_{\Omega} \sigma_{x} \varepsilon_{x}^{*} d \Omega=-\int_{\Gamma} m_{x} \frac{\partial w^{*}}{\partial x} \cos \alpha d \Gamma+\int_{\Omega}\left[\frac{\partial}{\partial x}\left(w^{*} \frac{\partial m_{x}}{\partial x}\right)-w^{*} \frac{\partial^{2} m_{x}}{\partial x^{2}}\right] d \Omega
$$

Depois, usando o teorema de Green, pode-se escrever:

$$
\int_{\Omega} \sigma_{x} \varepsilon_{x}^{*} d \Omega=\int_{\Gamma}\left(-m_{x} \frac{\partial w^{*}}{\partial x} \cos \alpha+w^{*} \frac{\partial m_{x}}{\partial x} \cos \alpha\right) d \Gamma-\int_{\Omega} w^{*} \frac{\partial^{2} m_{x}}{\partial x^{2}} d \Omega .
$$

Seguindo um procedimento similar, podemos mostrar que:

$$
\int_{\Omega} \sigma_{y} \varepsilon_{y}^{*} d \Omega=\int_{\Gamma}\left(-m_{y} \frac{\partial w^{*}}{\partial y} \sin \alpha+w^{*} \frac{\partial m_{y}}{\partial y} \sin \alpha\right) d \Gamma-\int_{\Omega} w^{*} \frac{\partial^{2} m_{y}}{\partial y^{2}} d \Omega
$$

e

$$
\begin{gathered}
\int_{\Omega} \tau_{x y} \gamma_{x y}^{*} d \Omega=\int_{\Gamma}\left(-m_{x y} \frac{\partial w^{*}}{\partial y} \cos \alpha-m_{x y} \frac{\partial w^{*}}{\partial x} \sin \alpha+w^{*} \frac{\partial m_{x y}}{\partial x} \sin \alpha+\right. \\
\left.w^{*} \frac{\partial m_{x y}}{\partial y} \cos \alpha\right) d \Gamma-\int_{\Omega} 2 w^{*} \frac{\partial^{2} m_{x y}}{\partial x \partial y} d \Omega
\end{gathered}
$$

Assim, a Equação (5.3) é escrita como:

$$
\begin{gathered}
\int_{\Omega} \sigma_{i j} \varepsilon_{i j}^{*} d \Omega=-\int_{\Gamma}\left(m_{x} \frac{\partial w^{*}}{\partial x} \cos \alpha+m_{y} \frac{\partial w^{*}}{\partial y} \sin \alpha+m_{x y} \frac{\partial w^{*}}{\partial y} \cos \alpha+\right. \\
\left.m_{x y} \frac{\partial w^{*}}{\partial x} \sin \alpha\right) d \Gamma+\int_{\Gamma} w^{*}\left[\left(\cos \alpha \frac{\partial m_{x}}{\partial x}+\frac{\partial m_{x y}}{\partial y}\right)\left(\sin \alpha \frac{\partial m_{y}}{\partial y}+\frac{\partial m_{x y}}{\partial x}\right)\right] d \Gamma- \\
\int_{\Gamma} w^{*}\left(\frac{\partial^{2} m_{x}}{\partial x^{2}}+2 \frac{\partial^{2} m_{x y}}{\partial x \partial y}+\frac{\partial^{2} m_{y}}{\partial y^{2}}\right) d \Gamma
\end{gathered}
$$

Substituindo as Equações (4.25) e (3.5) e usando a Equação (4.39), a Equação (5.13) pode ser escrita como:

$$
\begin{gathered}
\int_{\Omega} \sigma_{i j} \varepsilon_{i j}^{*} d \Omega=-\int_{\Gamma}\left(m_{x} \frac{\partial w^{*}}{\partial x} \cos \alpha+m_{y} \frac{\partial w^{*}}{\partial y} \sin \alpha+m_{x y} \frac{\partial w^{*}}{\partial y} \cos \alpha+\right. \\
\left.m_{x y} \frac{\partial w^{*}}{\partial x} \sin \alpha\right) d \Gamma+\int_{\Gamma} w^{*} q_{n} d \Gamma+\int_{\Omega} g w^{*} d \Omega .
\end{gathered}
$$

Da relação entre dois sistemas de coordenadas $(x, y)$ e $(n, s)$ tem-se: 


$$
\begin{gathered}
\frac{\partial w^{*}}{\partial x}=\frac{\partial w^{*}}{\partial n} \cos \alpha-\frac{\partial w^{*}}{\partial s} \sin \alpha, \\
\frac{\partial w^{*}}{\partial y}=\frac{\partial w^{*}}{\partial n} \sin \alpha+\frac{\partial w^{*}}{\partial s} \cos \alpha .
\end{gathered}
$$

Substituindo as Equações (5.15) na Equação (5.14) tem-se:

$$
\begin{aligned}
& \int_{\Omega} \sigma_{i j} \varepsilon_{i j}^{*} d \Omega=\int_{\Gamma}\left[m_{x} \cos \alpha\left(\frac{\partial w^{*}}{\partial n} \cos \alpha-\frac{\partial w^{*}}{\partial s} \sin \alpha\right)+\right. \\
& m_{y} \sin \alpha\left(\frac{\partial w^{*}}{\partial n} \sin \alpha+\frac{\partial w^{*}}{\partial s} \cos \alpha\right)+m_{x y} \cos \alpha\left(\frac{\partial w^{*}}{\partial n} \sin \alpha+\frac{\partial w^{*}}{\partial s} \cos \alpha\right)+ \\
& \left.m_{x y} \sin \alpha\left(\frac{\partial w^{*}}{\partial n} \cos \alpha-\frac{\partial w^{*}}{\partial s} \sin \alpha\right)\right] d \Gamma+\int_{\Gamma} w^{*} q_{n} d \Gamma+\int_{\Omega} g w^{*} d \Omega
\end{aligned}
$$

Depois de algumas manipulações algébricas, a Equação (5.16) pode ser reescrita como:

$$
\begin{gathered}
\int_{\Omega} \sigma_{i j} \varepsilon_{i j}^{*} d \Omega=\int_{\Gamma}\left\{\frac{\partial w^{*}}{\partial n}\left(m_{x} \cos ^{2} \alpha+m_{y} \sin ^{2} \alpha+2 m_{x y} \sin \alpha \cos \alpha\right)+\right. \\
\left.\frac{\partial w^{*}}{\partial s}\left[m_{x y}\left(\cos ^{2} \alpha-\sin ^{2} \alpha\right)+\left(m_{y}-m_{x}\right) \sin \alpha \cos \alpha\right]\right\} d \Gamma+ \\
\int_{\Gamma} w^{*} q_{n} d \Gamma+\int_{\Omega} g w^{*} d \Omega .
\end{gathered}
$$

Substituindo as Equações (4.37) e (4.38) na Equação (5.17), tem-se:

$$
\int_{\Omega} \sigma_{i j} \varepsilon_{i j}^{*} d \Omega=\int_{\Gamma}\left(m_{n} \frac{\partial w^{*}}{\partial n}+m_{n s} \frac{\partial w^{*}}{\partial s}-q_{n} w^{*}\right) d \Gamma+\int_{\Omega} g w^{*} d \Omega .
$$

Calculando o segundo termo da primeira integral do lado direito da Equação (5.18), temos:

$$
\int_{\Gamma} m_{n s} \frac{\partial w^{*}}{\partial s} d \Gamma=\left.m_{n s} w^{*}\right|_{\Gamma_{1}} ^{\Gamma_{2}}-\int_{\Gamma} \frac{\partial m_{n s}}{\partial s} w^{*} d \Gamma,
$$

onde $\Gamma_{1}$ e $\Gamma_{2}$ são as coordenadas dos extremos do contorno onde a integração está sendo realizada.

No caso de um contorno fechado sem canto, isto é, a função que descreve a curva de contorno e suas derivadas são contínuas, o primeiro termo do lado direito da Equação (5.19) desaparece. No caso onde há cantos, a Equação (5.19) pode ser escrita como: 


$$
\int_{\Gamma} m_{n s} \frac{\partial w^{*}}{\partial s} d \Gamma=-\sum_{i=1}^{N_{c}} R_{c_{i}} w_{c_{i}}^{*}-\int_{\Gamma} \frac{\partial m_{n s}}{\partial s} w^{*} d \Gamma
$$

onde

$$
R_{c_{i}}=m_{n s_{i}}^{+}-m_{n s_{i}}^{-}
$$

e os termos $w_{c_{i}}, m_{n s_{i}}^{+}, m_{n s_{i}}^{-}$são, respectivamente, os valores de deslocamentos e momentos depois e antes do canto $i$ da placa (Figura 5.2), $N_{c}$ é o número total de cantos no contorno, conforme Paiva (1987).
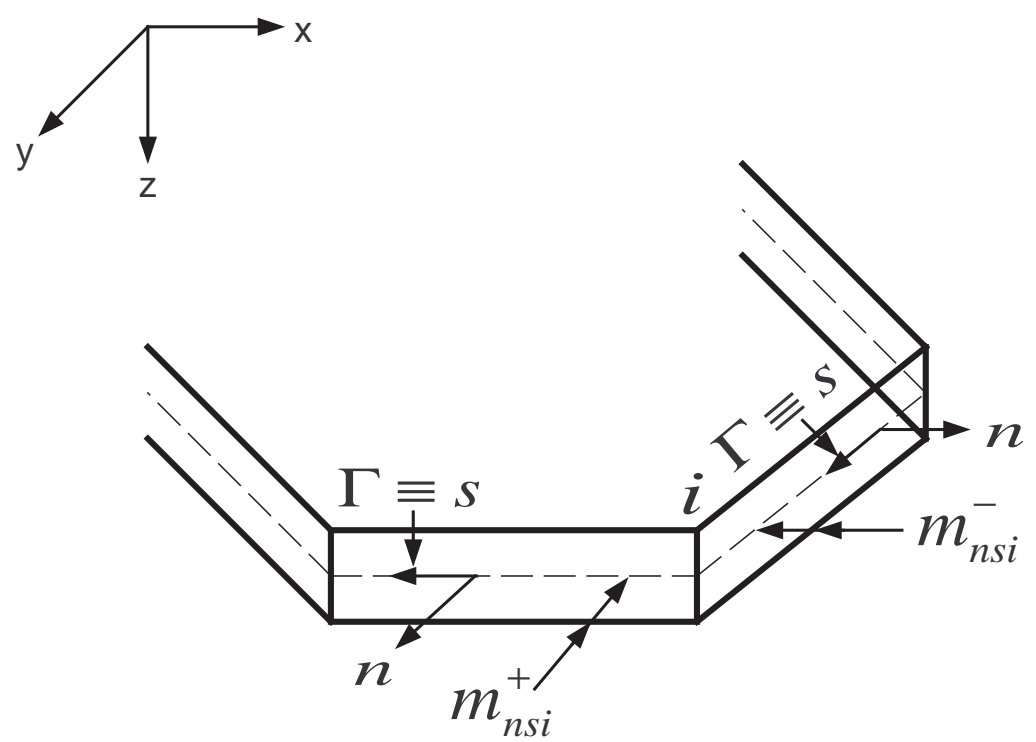

Figura 5.2: Canto $i$ do contorno da placa.

Das Equações (5.18) e (5.20), pode-se escrever:

$$
\int_{\Omega} \sigma_{i j} \varepsilon_{i j}^{*} d \Omega=\int_{\Gamma}\left(q_{n} w^{*}-m_{n} \frac{\partial w^{*}}{\partial n}+\frac{\partial m_{n s}}{\partial s}\right) d \Gamma+\sum_{i=1}^{N_{c}} R_{c_{i}} w_{c_{i}}^{*}+\int_{\Omega} g w^{*} d \Omega .
$$

Das Equações (5.22) e (4.40), tem-se:

$$
\int_{\Omega} \sigma_{i j} \varepsilon_{i j}^{*} d \Omega=\int_{\Gamma}\left(V_{n} w^{*}-m_{n} \frac{\partial w^{*}}{\partial n}\right) d \Gamma+\sum_{i=1}^{N_{c}} R_{c_{i}} w_{c_{i}}^{*}+\int_{\Omega} g w^{*} d \Omega .
$$

Seguindo um procedimento similar àquele usado para obter a Equação (5.23), o lado esquerdo da Equação (5.1) pode ser escrito como:

$$
\int_{\Omega} \sigma_{i j}^{*} \varepsilon_{i j} d \Omega=\int_{\Gamma}\left(V_{n}^{*} w-m_{n} \frac{\partial w^{*}}{\partial n}\right) d \Gamma+\sum_{i=1}^{N_{c}} R_{c_{i}}^{*} w_{c_{i}}+\int_{\Omega} g^{*} w d \Omega .
$$


Substituindo as Equações (5.23) e (5.24) na Equação (5.1), pode-se escrever:

$$
\begin{array}{r}
\int_{\Gamma}\left(V_{n} w^{*}-m_{n} \frac{\partial w^{*}}{\partial n}\right) d \Gamma+\sum_{i=1}^{N_{c}} R_{c_{i}} w_{c_{i}}^{*}+\int_{\Omega} g w^{*} d \Omega= \\
\int_{\Gamma}\left(V_{n}^{*} w-m_{n} \frac{\partial w^{*}}{\partial n}\right) d \Gamma+\sum_{i=1}^{N_{c}} R_{c_{i}}^{*} w_{c_{i}}+\int_{\Omega} g^{*} w d \Omega .
\end{array}
$$

A Equação (5.25) relaciona dois estados de um material elástico. Para aplicar esta equação para resolver problemas de flexão, precisamos considerar um dos estados como conhecido e o outro como o estado que queremos analisar. Para obter a equação integral de contorno, o estado conhecido é ajustado para que a integral de domínio dada por:

$$
\int_{\Omega} g^{*} w d \Omega
$$

desapareça. Usando as propriedades da função delta de Dirac $\delta(P, Q)$, de forma que $g^{*}=\delta(P, Q)$, a integral (5.26) é escrita como:

$$
\int_{\Omega} \delta(P, Q) w(P) d \Omega(P)=w(Q)
$$

onde $Q$ é o ponto onde a carga é aplicada, conhecido como ponto fonte, e $P$ é o ponto onde o deslocamento é observado, conhecido como ponto campo. O estado correspondente a um material linear sob carregamento de uma função delta de Dirac é conhecido como um estado fundamental e as variáveis da Equação (5.25) relacionadas a este estado $\left(w^{*}, V_{n}^{*}\right.$ e $\left.m_{n}^{*}\right)$ são conhecidas como soluções fundamentais, as quais são calculadas analiticamente a partir da Equação (4.42).

Considerando o estado "*"como o estado fundamental, a Equação (5.25) pode ser escrita como:

$$
\begin{gathered}
K w(Q)+\int_{\Gamma}\left[V_{n}^{*}(Q, P) w(P)-m_{n}^{*}(Q, P) \frac{\partial w(P)}{\partial n}\right] d \Gamma(P)+\sum_{i=1}^{N_{c}} R_{c_{i}}^{*}(Q, P) w_{c_{i}}(P)- \\
\int_{\Gamma}\left[V_{n}(P) w^{*}(Q, P)-m_{n}(P) \frac{\partial w^{*}}{\partial n}(Q, P)\right] d \Gamma(P)+\sum_{i=1}^{N_{c}} R_{c_{i}}(P) w_{c_{i}}^{*}(Q, P)+ \\
\int_{\Omega} g(P) w^{*}(Q, P) d \Omega .
\end{gathered}
$$

A Equação (5.28) é a equação de placas finas para deslocamentos em pontos do domínio da placa. Esta equação fornece deslocamentos em todos os pontos do domínio da placa a partir das cortantes equivalentes $\left(V_{n}\right)$, momentos de flexão na direção normal 
$\left(m_{n}\right)$, reação de canto $\left(R_{c_{i}}\right)$, deslocamentos $(w)$ e rotações em relação à normal $(\partial w / \partial n)$ conhecidos no contorno.

A constante $K$ é introduzida para se considerar que a função delta de Dirac pode ser aplicada no domínio, no contorno ou fora do domínio. Se a função delta de Dirac é aplicada em um ponto onde o contorno é suave, então $K=1 / 2$. As variáveis da equação (5.28) são deslocamentos $w(P)$, rotações $\frac{\partial w(P)}{\partial n}$, momentos $m_{n}(P)$, e forças $V_{n}(P)$. Para uma dada condição de contorno, algumas destas variáveis são conhecidas e outras desconhecidas. Para se ter um número de equações igual ao número de variáveis desconhecidas, é necessário escrever a equação integral correspondente a derivada do deslocamento $w(Q)$ em relação ao sistema de coordenadas cartesiano fixo no ponto de origem, isto é, o ponto onde o delta de Dirac do estado fundamental é aplicado. As direções dos eixos deste sistema de coordenadas são coincidentes com as direções normal e a tangente ao contorno no ponto fonte. Para problemas de flexão em placas isotrópicas tem-se que a equação integral de contorno escrita em termos de quatro valores de contorno básicos, isto é, deflexão $w$, inclinação da normal $\partial w / \partial n$, força cortante $V_{n}$ e momento fletor $m_{n}$. Em um problema bem colocado dois destes quatro valores são incógnitas do problema e dois são condições de contorno conhecidas.

Pode-se verificar que num problema de flexão em placas há sempre duas incógnitas a serem determinadas em qualquer ponto do contorno e consequentemente, a solução do problema requer que uma segunda equação seja estabelecida.

A segunda equação integral de contorno é obtida da derivada da Equação (5.28) em relação à direção $n_{1}$ normal ao contorno no ponto fonte e também corresponde à solução do binário unitário.

$$
\begin{aligned}
& \frac{1}{2} \frac{\partial w(Q)}{\partial n_{1}}+\int_{\Gamma}\left[\frac{\partial V^{*}}{\partial n_{1}}(Q, P) w(P)-\frac{\partial m_{n}^{*}}{\partial n_{1}}(Q, P) \frac{\partial w}{\partial n}(P)\right] d \Gamma(P)+ \\
& \sum_{i=1}^{N_{c}} \frac{\partial R^{*} c_{i}}{\partial n_{1}}(Q, P) w_{c_{i}}(P)=\int_{\Gamma}\left\{V_{n}(P) \frac{\partial w^{*}}{\partial n_{1}}(Q, P)-m_{n}(P) \frac{\partial}{\partial n_{1}}\left[\frac{\partial w^{*}}{\partial n_{1}}(Q, P)\right]\right\} d \Gamma(P)+ \\
& \sum_{i=1}^{N_{c}} R_{c_{i}}(P) \frac{\partial w_{c_{i}}^{*}}{\partial n_{1}}(Q, P)+\int_{\Omega} g(P) \frac{\partial w^{*}}{\partial n_{1}}(Q, P) d \Omega .
\end{aligned}
$$

É importante dizer que é possível usar apenas a Equação (5.28) em uma formulação de elementos de contorno usando como pontos fonte os nós do contorno e um número igual de pontos externos ao domínio do problema. 


\subsection{Equação Matricial}

Com o objetivo de calcular as variáveis de contorno desconhecidas, o contorno $\Gamma$ é discretizado em $N_{e}$ elementos e as variáveis de contorno $w, \partial w / \partial n, m_{n}$ e $V_{n}$ são assumidas com uma variação constante ao longo de cada elemento. Tomando um nó $d$ como o ponto fonte, as equações (5.28) e (5.29) podem ser escritas na forma matricial como:

$$
\begin{aligned}
& \frac{1}{2}\left\{\begin{array}{c}
w^{(d)} \\
\frac{\partial w^{(d)}}{\partial n_{1}}
\end{array}\right\}+\sum_{i=1}^{N E}\left(\left[\begin{array}{ll}
H_{11}^{(i, d)} & H_{12}^{(i, d)} \\
H_{21}^{(i, d)} & H_{22}^{(i, d)}
\end{array}\right]\left\{\begin{array}{c}
w^{(i)} \\
\frac{\partial w^{(i)}}{\partial n}
\end{array}\right\}\right)= \\
& \sum_{i=1}^{N E}\left(\left[\begin{array}{ll}
G_{11}^{(i, d)} & G_{12}^{(i, d)} \\
G_{21}^{(i, d)} & G_{22}^{(i, d)}
\end{array}\right]\left\{\begin{array}{c}
V_{n}^{(i)} \\
m_{n}^{(i)}
\end{array}\right\}\right)+\sum_{i=1}^{N C}\left(\left\{\begin{array}{c}
C_{1}^{(i, d)} \\
C_{2}^{(i, d)}
\end{array}\right\} w_{c}^{(i)}\right)+ \\
& \sum_{i=1}^{N C}\left(\left\{\begin{array}{l}
F_{1}^{(i, d)} \\
F_{2}^{(i, d)}
\end{array}\right\} R_{c}^{(i)}\right)+\left\{\begin{array}{c}
P_{1} \\
P_{2}
\end{array}\right\}
\end{aligned}
$$

onde $N C$ são números de cantos. Os termos da equação (5.30) são integrais dadas por:

$$
\begin{array}{rlrl}
H_{11}^{(i, d)} & =\int_{\Gamma_{i}} V_{n}^{*} d \Gamma, & H_{12}^{(i, d)} & =-\int_{\Gamma_{i}} m_{n}^{*} d \Gamma, \\
H_{21}^{(i, d)}=\int_{\Gamma_{i}} \frac{\partial V_{n}^{*}}{\partial n_{1}} d \Gamma, & H_{22}^{(i, d)}=-\int_{\Gamma_{i}} \frac{\partial m_{n}^{*}}{\partial n_{1}} d \Gamma, \\
G_{11}^{(i, d)}=\int_{\Gamma_{i}} w^{*} d \Gamma, & G_{12}^{(i, d)}=-\int_{\Gamma_{i}} \frac{\partial w^{*}}{\partial n} d \Gamma, \\
G_{21}^{(i, d)}=\int_{\Gamma_{i}} \frac{\partial w^{*}}{\partial n_{1}} d \Gamma, & G_{22}^{(i, d)}=-\int_{\Gamma_{i}} \frac{\partial}{\partial n_{1}} \frac{\partial m_{n}^{*}}{\partial n_{1}} d \Gamma, \\
C_{1}^{(i, d)}=R_{c}^{*}, & C_{2}=\frac{\partial R_{c}^{*}}{\partial n_{1}}, \\
P_{1}=\int_{\Gamma_{g}} F^{*} d \Gamma, & P_{2}=\int_{\Gamma_{g}} G^{*} d \Gamma .
\end{array}
$$

As integrais observadas acima, podem ser definidas como: fracas, fortes ou hipersingulares, quando o elemento que esta sendo integrado localiza-se no ponto fonte. Neste trabalho, estas integrais são tratados analiticamente, como mostrado por Paiva et al. (2003).

A equação matricial (5.30) tem duas equações e $2 N E+N C$ variáveis desconhecidas. Para se obter um sistema linear solucionável, o ponto fonte é colocado sucessivamente 
em cada nó do contorno $d=1, \ldots, N E$ bem como em cada nó de canto $d=N E+$ $1, \ldots, N E+N C$. É importante notar que enquanto ambas as equações, (5.28) e (5.29), são usadas para cada nó de contorno (fornecendo as primeiras $2 N E$ equações), somente a equação (5.28) é usada para cada canto (fornecendo outras $N C$ equações). Então, a seguinte é obtida:

$$
\left[\begin{array}{cc}
\mathbf{H}^{\prime} & \mathbf{R}^{\prime} \\
\mathbf{H}^{\prime \prime} & \mathbf{R}^{\prime \prime}
\end{array}\right]\left\{\begin{array}{c}
\mathbf{w}_{b n} \\
\mathbf{w}_{c}
\end{array}\right\}=\left[\begin{array}{cc}
\mathbf{G}^{\prime} & \mathbf{C}^{\prime} \\
\mathbf{G}^{\prime \prime} & \mathbf{C}^{\prime \prime}
\end{array}\right]\left\{\begin{array}{ll}
\mathbf{V}_{b n} & \mathbf{V}_{c}
\end{array}\right\}+\left\{\begin{array}{c}
\mathbf{P}_{b n} \\
\mathbf{P}_{c}
\end{array}\right\}
$$

onde, $\mathbf{w}_{b n}$ contém o deslocamento transversal e a rotação de cada nó de contorno, $\mathbf{V}_{b n}$ contém a força cisalhante e o momento torsor de cada nó de contorno, $\mathbf{P}_{b n}$ contém a integral de domínio para cada nó de contorno, $\mathbf{w}_{c}$ contém o deslocamento transversal de cada canto, $\mathbf{V}_{c}$ contém a reação de canto para cada canto, $\mathbf{P}_{c}$ contém a integral de domínio para cada canto. Os termos $\mathbf{H}^{\prime}, \mathbf{C}^{\prime}, \mathbf{R}^{\prime}$ e $\mathbf{G}^{\prime}$ são matrizes que contém os respectivos termos da equação (5.30) escritos para os $N_{e}$ nós de contorno. Os termos $\mathbf{H}^{\prime \prime}, \mathbf{C}^{\prime \prime}, \mathbf{R}^{\prime \prime}$ e $\mathbf{G}^{\prime \prime}$ são matrizes que contém os respectivos primeiros termos da equação (5.30) escrita para os $N_{c}$ cantos.

A equação (5.37) pode ser reescrita como:

$$
\mathbf{H w}=\mathbf{G V}+\mathbf{P}
$$

onde,

$$
\begin{gathered}
\mathbf{H}=\left[\begin{array}{ll}
\mathbf{H}^{\prime} & \mathbf{R}^{\prime} \\
\mathbf{H}^{\prime \prime} & \mathbf{R}^{\prime \prime}
\end{array}\right], \\
\mathbf{w}=\left\{\begin{array}{l}
\mathbf{w}_{b n} \\
\mathbf{w}_{c}
\end{array}\right\}, \\
\mathbf{G}=\left[\begin{array}{ll}
\mathbf{G}^{\prime} & \mathbf{C}^{\prime} \\
\mathbf{G}^{\prime \prime} & \mathbf{C}^{\prime \prime}
\end{array}\right], \\
\mathbf{V}=\left\{\begin{array}{c}
\mathbf{V}_{b n} \\
\mathbf{V}_{c}
\end{array}\right\}, \\
\mathbf{P}=\left\{\begin{array}{c}
\mathbf{P}_{b n} \\
\mathbf{P}_{c}
\end{array}\right\} .
\end{gathered}
$$

Aplicando as condições de contorno, a equação (5.37) pode ser rearranjada como:

$$
\mathrm{Ax}=\mathrm{b}
$$

que pode ser resolvida pelo procedimento padrão para sistemas lineares. 


\section{Capítulo 6}

\section{Transformação das Integrais de Domínio em Integrais de Contorno}

\subsection{Introdução}

A aplicação do método dos elementos de contorno requer, preferencialmente, que a solução fundamental para o problema em consideração seja conhecida. Essa solução fundamental deve levar em conta todos os termos da equação governante de forma a obter uma formulação onde apenas o contorno é discretizado. Quando isso não for possível, os termos não considerados na obtenção da solução fundamental produzirão integrais de domínio que preferencialmente, devem ser transformadas em integrais de contorno. A primeira alternativa é fazer a transformação exata da integral de domínio em integral de contorno. Pórem, isto só é possível quando os termos não considerados são funções apenas da geometria (carregamento distribuído, por exemplo). A segunda alternativa é transferir os efeitos da integral de domínio para o contorno usando-se o método de elementos de contorno de integração radial. A terceira alternativa é transformar os efeitos da integral de domínio para o contorno usando-se integração analítica. No caso deste trabalho, as cargas distribuídas serão transformadas por transformação exata, enquanto que os termos de inércia serão transformados usando o método de integração radial.

A força de corpo é representada pela equação abaixo:

$$
b=g+\varrho h \ddot{w}
$$

onde $g$ denota a carga distribuída e $\varrho$ w representa o termo de inércia, sendo $h$ a espessura e $\varrho$ a densidade do material. 


\subsection{Transformação Exata}

Como pôde ser observado nas Equações (5.28) e (5.29), há integrais de domínio na formulação devido a carga distribuída no domínio e aos termos de inércia. Estas integrais podem ser calculadas por integração direta, através de células na área $\Omega_{g}$, Figura 4.1. Contudo, a formulação dos elementos de contorno perde seu principal atrativo que é a discretização somente do contorno. Neste trabalho, as integrais de domínio oriundas das cargas distribuídas são transformadas em integrais de contorno por uma transformação exata.

Considere a placa da Figura 4.1 sob um carregamento $g$ aplicado em uma área $\Omega_{g}$. Assumindo que o carregamento $g$ tem uma distribuição linear $(A x+B y+C)$ na área $\Omega_{g}$, a integral de domínio pode ser escrita como:

$$
\int_{\Omega_{g}} g w^{*} d \Omega=\int_{\Omega_{g}}(A x+B y+C) w^{*} \rho d \rho d \theta,
$$

$\mathrm{Ou}$

$$
\int_{\Omega_{g}} g w^{*} d \Omega=\int_{\theta} \int_{0}^{r}(A x+B y+C) w^{*} \rho d \rho d \theta,
$$

onde, $r$ é o valor de $\rho$ em um ponto do contorno $\Gamma_{g}$.

Definindo $F^{*}$ como a seguinte integral:

$$
F^{*}=\int_{0}^{r}(A x+B y+C) w^{*} \rho d \rho
$$

pode-se escrever:

$$
\int_{\Omega_{g}} g w^{*} d \Omega=\int_{\theta} F^{*} d \theta
$$

Considerando um ângulo infinitesimal $d \theta$, Figura 6.1, a relação entre o comprimento do arco $r d \theta$ e o comprimento infinitesimal do contorno $d \Gamma$, pode ser escrito como:

$\mathrm{Ou}$

$$
\cos \alpha=\frac{r \frac{d \theta}{2}}{\frac{d \Gamma}{2}}
$$

$$
d \theta=\frac{\cos \alpha}{r} d \Gamma .
$$

Usando as propriedades do produto interno dos vetores unitários $\mathbf{n}$ e $\mathbf{r}$, indicados na Figura 6.1, podemos escrever:

$$
d \theta=\frac{\mathbf{n} \cdot \mathbf{r}}{r} d \Gamma
$$




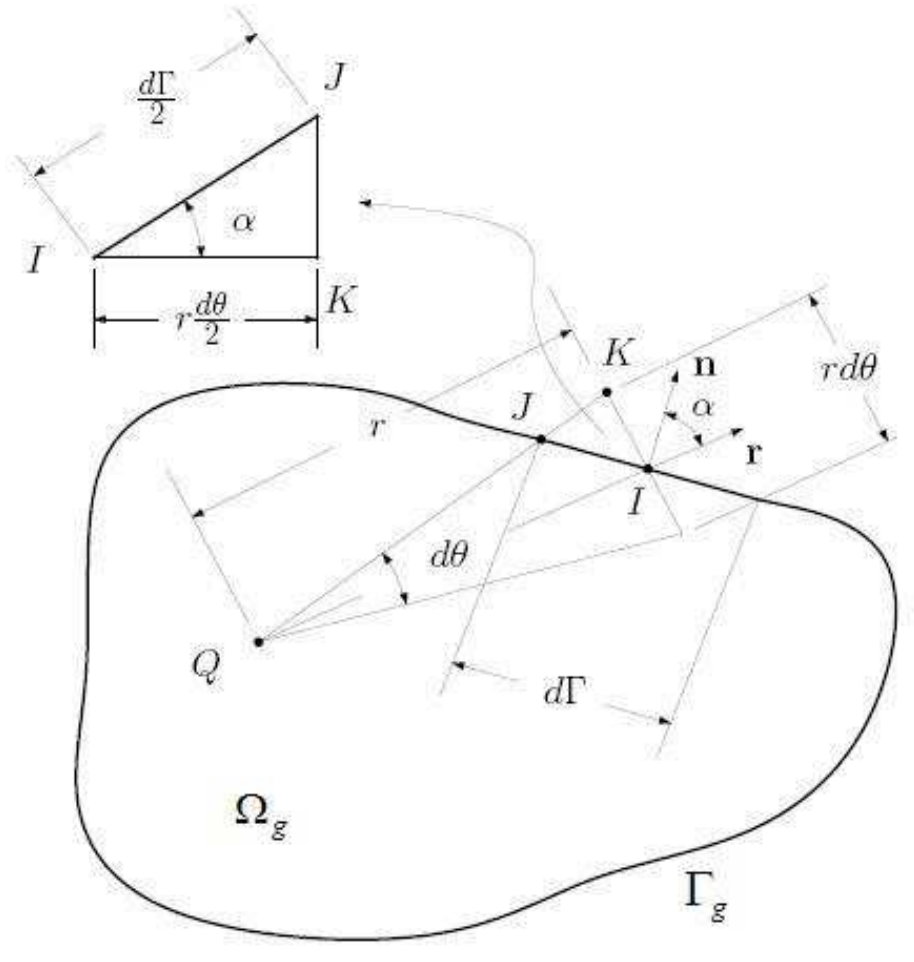

Figura 6.1: Transformação da integral de domínio em integral de contorno.

Finalmente, substituindo a Equação (6.8) na Equação (6.5), a integral de domínio da Equação (5.28) pode ser escrita como uma integral de contorno dada por:

$$
\int_{\Omega_{g}} g w^{*} d \Omega=\int_{\Gamma_{g}} \frac{F^{*}}{r} \mathbf{n} \cdot \mathbf{r} d \Gamma .
$$

Sabendo que

$$
x=\rho \cos \theta
$$

e

$$
y=\rho \sin \theta,
$$

O termo de domínio da Equação (5.29) pode ser escrito como:

$$
\int_{\Omega_{g}} g \frac{\partial w^{*}}{\partial n_{1}} d \Omega=\int_{\theta} G^{*} d \theta
$$

onde

$$
G^{*}=\int_{0}^{r}(A x+B y+C) \frac{\partial w^{*}}{\partial n_{1}} \rho d \rho
$$




\subsection{Método da integração radial - RIM}

O RIM aproxima a força de corpo $b$ como uma soma de $M$ produtos de funções de aproximação $f^{m}$ e coeficientes a determinar $\gamma^{m}$, ou seja:

$$
b(P)=\sum_{m=1}^{M} \gamma^{m} f^{m}
$$

para as funções de aproximação baseadas puramente em funções de base radiais, ou:

$$
b(P)=\sum_{m=1}^{M} \gamma^{m} f^{m}+a x+b y+c
$$

$\mathrm{e}$

$$
\sum_{m=1}^{M} \gamma^{m} x_{m}=\sum_{m=1}^{M} \gamma^{m} y_{m}=\sum_{m=1}^{M} \gamma^{m}=0
$$

para as funções de aproximação expandidas por polinômios.

A integral de domínio da equação (5.28) pode ser escrita como:

$$
P_{1}(Q)=\sum_{m=1}^{M} \gamma^{m} \int_{\Omega_{g}} f^{m} w^{*}(Q, P) \rho d \rho d \theta
$$

$\mathrm{Ou}$

$$
P_{1}(Q)=\sum_{m=1}^{M} \gamma^{m} \int_{\theta} \int_{0}^{r} f^{m} w^{*}(Q, P) \rho d \rho d \theta,
$$

onde $r$ é o valor de $\rho$ em um ponto no contorno $\Gamma_{g}$ (ver Figura 6.1):

Definindo $F^{m}(Q)$ como:

$$
F^{m}(Q)=\int_{0}^{r} f^{m} w^{*}(Q, P) \rho d \rho
$$

pode-se escrever:

$$
P_{1}(Q)=\sum_{m=1}^{M} \gamma^{m} \int_{\theta} F^{m}(Q) d \theta
$$

Substituindo a equação (6.8) na equação (6.20), a equação integral de domínio da equação (5.28) pode ser escrita na seguinte integral de contorno dada por:

$$
P_{1}(Q)=\sum_{m=1}^{M} \gamma^{m} \int_{\Gamma_{g}} \frac{F^{m}(Q)}{r} \mathbf{n} \cdot \mathbf{r} d \Gamma .
$$

Seguindo procedimentos similares aos usados para obter a equação (6.21), o termo de domínio da equação pode ser escrito assim: 


$$
P_{2}(Q)=\int_{\Omega_{g}} b \frac{\partial w^{*}(Q, P)}{\partial n_{1}} d \Omega=\sum_{m=1}^{M} \gamma^{m} \int_{\Gamma_{g}} \frac{G^{m}(Q)}{r} \mathbf{n} \cdot \mathbf{r} d \Gamma
$$

onde

$$
G^{m}(Q)=\int_{0}^{r} f^{m} \frac{\partial w^{*}(Q, P)}{\partial n_{1}} \rho d \rho .
$$

Assim, a integral de domínio da equação (5.30) pode ser escrita como:

$$
\left\{\begin{array}{c}
P_{1}^{(d)} \\
P_{2}^{(d)}
\end{array}\right\}=\sum_{m=1}^{M} \gamma^{m} \sum_{i=1}^{N_{e}}\left\{\begin{array}{c}
p_{1}^{(d, m)} \\
p_{2}^{(d, m)}
\end{array}\right\}
$$

onde

$$
p_{1}^{(d, m)}=\int_{\Gamma_{i}} \frac{F^{(d, m)}}{r} \mathbf{n} \cdot \mathbf{r} d \Gamma, \quad p_{2}^{(d, m)}=\int_{\Gamma_{i}} \frac{G^{(d, m)}}{r} \mathbf{n} \cdot \mathbf{r} d \Gamma .
$$

ou, na forma matricial, como:

$$
P(Q)=\left[\begin{array}{llll}
\int_{\Gamma} \frac{F_{1}(Q)}{r} \mathbf{n} \cdot \mathbf{r} d \Gamma & \int_{\Gamma} \frac{F_{2}(Q)}{r} \mathbf{n} \cdot \mathbf{r} d \Gamma & \ldots & \int_{\Gamma} \frac{F_{M}(Q)}{r} \mathbf{n} \cdot \mathbf{r} d \Gamma
\end{array}\right]\left\{\begin{array}{c}
\gamma_{1} \\
\gamma_{2} \\
\vdots \\
\gamma_{M}
\end{array}\right\}
$$

Para calcular $\gamma_{m}$, é necessário considerar a força de corpo em $M$ pontos do domínio e do contorno. No caso deste trabalho, estes pontos são os nós do contorno e alguns pontos internos. Assim, a equação (6.14) pode ser escrita como:

$$
\mathbf{b}=\mathbf{F} \gamma
$$

e $\gamma$ pode ser calculado como:

$$
\gamma=\mathbf{F}^{-1} \mathbf{b}
$$

Substituindo (6.28) na equação (6.26), tem-se:

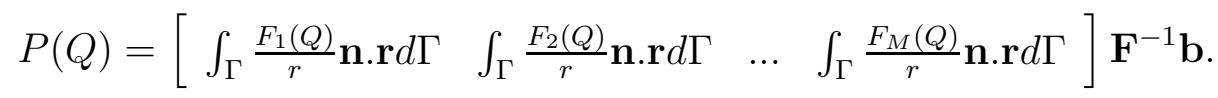

Escrevendo a equação (6.29) para todos os pontos do domínio, isto é, todos os nós do contorno e pontos internos, tem-se: 


$$
\mathbf{P}=\mathbf{R F}^{-1} \mathbf{b}=\mathbf{S b}
$$

onde $\mathbf{S}=\mathbf{R F}^{-1}, \mathbf{P}$ é um vetor que contém os valores de $P(Q)$ em todos os pontos fontes $Q$ e $\mathbf{R}$ é uma matriz que contém todos os valores das integrais (6.29) quando esta equação é escrita para todos os pontos fontes $Q$.

As funções de aproximação $f^{m}$ serão funções de base radial escritas em termos de $R$, onde $R$ é a distância entre o centro $S$ da função de base radial e o ponto de integração $P$. Da Figura 6.2, pode-se escrever:

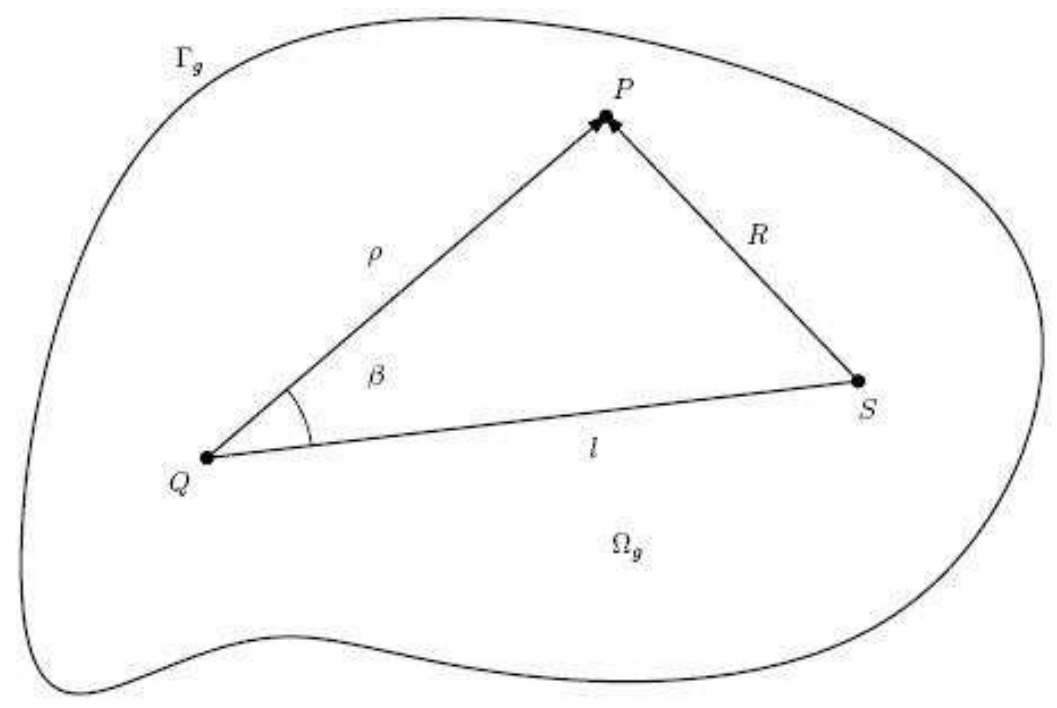

Figura 6.2: Posição dos pontos no domínio.

$$
R=\sqrt{\rho^{2}+l^{2}-2 \rho l \cos \beta}
$$

onde $l$ é a distância entre os pontos $S$ e $Q$ e $\beta$ é o ângulo entre $\rho$ e $l$.

Como consequência do uso das funções de aproximação, o custo computacional do RIM é alto, uma vez que, as integrais dadas pelas equações (6.19) e (6.23) não podem ser calculadas analiticamente para a maioria das funções de aproximação. Por exemplo, se $f^{m}=R$, a equação (6.19) é escrita como:

$$
F^{m}(Q)=\int_{0}^{r} c_{1} \sqrt{\rho^{2}+l^{2}-2 \rho l \cos \beta} \log \left(c_{2} \rho\right) \rho^{3} d \rho,
$$

onde $c_{1}$ e $c_{2}$ são coeficientes que não dependem de $\rho$. A integral da equação (6.32) não pode ser calculada analiticamente. O cálculo numérico desta integral torna mais alto o custo computacional do RIM, uma vez que no DRM não se faz nenhuma integração numérica na transformação da integral de domínio em integrais de contorno. 
A vantagem mais interessante do RIM sobre o DRM para formulações que envolvam materiais anisotrópicos é que as funções de aproximação $f_{m}$ podem ser escolhidas livremente, pois o RIM não usa as soluções particulares $\hat{w}_{m}$ obtidas da equação diferencial (4.34).

Estas soluções particulares são necessárias no DRM, o que restringe a escolha das funções de aproximação devido a complexidade da equação (4.34).

Neste trabalho foi usada como função de aproximação de base radial denominada spline de placas finas que é dada por:

$$
f_{m_{3}}=R^{2} \log (R)
$$

Alguns trabalhos da literatura Golberg et al. (1999) mostraram que esta função possui uma alta taxa de convergência quando usadas na forma aumentada por polinômios, ou seja, quando a aproximação da força de corpo é dada pelas equações (6.15) e (6.16). Neste caso ela é chamada de função spline de placas finas aumentada, ou ATPS (augmented thin plate spline).

\subsection{Método de Houbolt}

O método dos elementos de contorno de integração radial será aplicados ao cálculo dos campos de deslocamentos para problemas transientes no domínio do tempo. A integração no tempo será realizada utilizando o método proposto por Houbolt (1950) por já ter sido mostrado por Loeffler and Mansur (1987) que o método de Houbolt é o mais apropriado para se usar a integração direta no tempo junto com o método dos elementos de contorno de integração radial. Uma importante característica do método de Houbolt é que ele tem um alto amortecimento numérico. Este amortecimento torna os resultados obtidos pelo método dos elementos de contorno de integração radial mais suaves que os obtidos por outras formulações do método dos elementos de contorno aplicadas à elasto-dinâmica, Fedelinsk et al. (1996) e Chirino et al. (1994). Porém, o amortecimento numérico pode ser reduzido usando-se passos de tempo menores. Algumas vezes, para se conseguir reduzir o passo de tempo e obter uma maior precisão dos resultados é necessário refinar a malha ou aumentar o número de nós internos.

Considere que as únicas forças de corpo presente num corpo de domínio $\Omega$ e contorno $\Gamma$ são devido ao campo de aceleração ü, ou seja:

$$
\mathbf{b}=\rho h \ddot{\mathbf{u}} .
$$

O esquema de Houbolt está enquadrado entre os métodos de múltiplos passos, pois o mesmo não se utiliza apenas dos valores do passo anterior para determinar os valores 
atuais e sim de três outros passos passados. Para se proceder a integração no tempo durante um período $\mathcal{T}$, este período é dividido em $N$ intervalos iguais $\Delta \tau(\mathcal{T}=N \Delta \tau)$. A aceleração em $\tau+\Delta \tau$ é aproximada pela expressão de diferenças finitas:

$$
\ddot{\mathbf{u}}_{\tau+\Delta \tau}=\frac{1}{\Delta \tau^{2}}\left(2 \mathbf{u}_{\tau+\Delta \tau}-5 \mathbf{u}_{\tau}+4 \mathbf{u}_{\tau-\Delta \tau}-\mathbf{u}_{\tau-2 \Delta \tau}\right) .
$$

onde: $\tau+\Delta \tau \rightarrow$ passo de tempo atual; $\tau \rightarrow$ passo de tempo imediatamente anterior ao passo atual $\tau+\Delta \tau ; \tau-\Delta \tau \rightarrow$ passo de tempo imediatamente anterior ao passo $\tau$; $\tau-2 \Delta \tau \rightarrow$ passo de tempo imediatamente anterior ao passo $\tau-\Delta \tau ; \Delta \tau \rightarrow$ intervalo de tempo entre passos consecutivos de tempo.

Desde que $\mathbf{u}_{\tau}, \mathbf{u}_{\tau-\Delta \tau}$ e $\mathbf{u}_{\tau-2 \Delta \tau}$ sejam conhecidos, pode-se calcular $\mathbf{u}_{\tau+\Delta \tau}$ através de um sistema, da forma:

$$
\mathbf{A x}_{\tau+\Delta \tau}=\mathbf{y}_{\tau+\Delta \tau}
$$

onde $\mathbf{x}_{\tau+\Delta \tau}$ é o vetor de variáveis desconhecidas e $\mathbf{y}_{\tau+\Delta \tau}$ é o vetor de variáveis conhecidas, sendo que seus elementos são calculados a partir dos valores de $\mathbf{u}$ dos passos de tempo anteriores e das condições de contorno no tempo $\tau+\Delta \tau$.

Uma vez que o sistema (6.36) é resolvido, o vetor $\mathbf{u}_{\tau+\Delta \tau}$ é conhecido e a solução pode então ser encontrada para o próximo passo de tempo. 


\section{Capítulo 7}

\section{Associação de Macro-elementos Via Sub-regiões do MEC}

\subsection{Introdução}

Neste capítulo, os aspectos finais da implementação serão abordados. Até o momento as duas formulações, de elasticidade plana e placas finas, foram apresentadas paralelamente ao longo dos capítulos 2 a 5. A contribuição deste trabalho decorre da associação das formulações anteriormente descritas sob o aspecto estático e dinâmico e, além disso, calcular o campo de deslocamento para problemas transientes no domínio do tempo desenvolvido por Hoefel (2006). As seções a seguir, tem a intenção de agrupar estas formulações em uma só e dar os detalhes finais a respeito da implementação da formulação para associação de placas no espaço.

A análise de estruturas tridimensionais formadas por elementos estruturais planos é feita admitindo-se que cada elemento que compõem essas estruturas seja analisado como um macro-elemento. A principal característica deste macro-elemento, é a superposição do estado plano de tensão e de flexão de placas finas.

As equações integrais de flexão de placas finas e estado plano de tensão em um macro-elemento são discretizadas em elementos de contorno constantes. Em seguida, a técnica de sub-regiões é aplicada no desenvolvimento da formulação para associação de macro-elementos no espaço. O sistema algébrico para a estrutura é obtido através da associação dos sistemas de equações de cada macro-elemento individual. A junção destes sistemas de equações é feita através de imposições de equilíbrio e compatibilidade de esforços e deslocamentos entre as interfaces (arestas comuns). 


\subsection{Formulação do macro-elemento.}

O objetivo desta seção é desenvolver um elemento estrutural plano que contenha simultaneamente comportamento de elasticidade plana (membrana) e de placa. $\mathrm{O}$ elemento plano, denominado macro-elemento, é obtido a partir da discretização das equações integrais de contorno desenvolvida ao longo desta tese. As equações integrais obtidas são transformadas, depois da discretização, em um sistema de equações lineares onde as incógnitas são deslocamentos, rotações, forças de superfície e momentos em nós do contorno, pontos internos e em cantos dos macro-elementos. Aplicando-se as condições de contorno e resolvendo o sistema de equações resultantes obtém-se as variáveis desconhecidas da estrutura formada pelos macro-elementos.

\subsubsection{Equações Algébricas do Macro-Elemento}

Seja $\Psi$ um ponto genérico de um macro-elemento, Tanaka et al. (1998), como apresentado na Figura 7.1. O campo de deslocamentos e esforços para o $\Psi$ é composto de quatro variáveis relacionadas à superposição dos estados plano de tensão e flexão de placas finas. Nas equações integrais da elasticidade plana, as variáveis do problema são os deslocamentos $u_{1}$ e $u_{2}$ e dois esforços de superfície $t_{1}$ e $t_{2}$. Nas equações integrais de placa, as variáveis relacionadas são $w, \frac{\partial w}{\partial n}, V_{n}$ e $M_{n}$ e são definidas no Capítulo 3. Uma vez que não existe graus de liberdade nos cantos na formulação de elasticidade plana, os cantos são considerados livres no macro elemento $\left(R_{c}=0\right)$. O vetor $\mathbf{u}$ é definido como o vetor de deslocamentos do macro-elemento em um ponto genérico $\Psi$ e seus termos são organizados da seguinte forma:

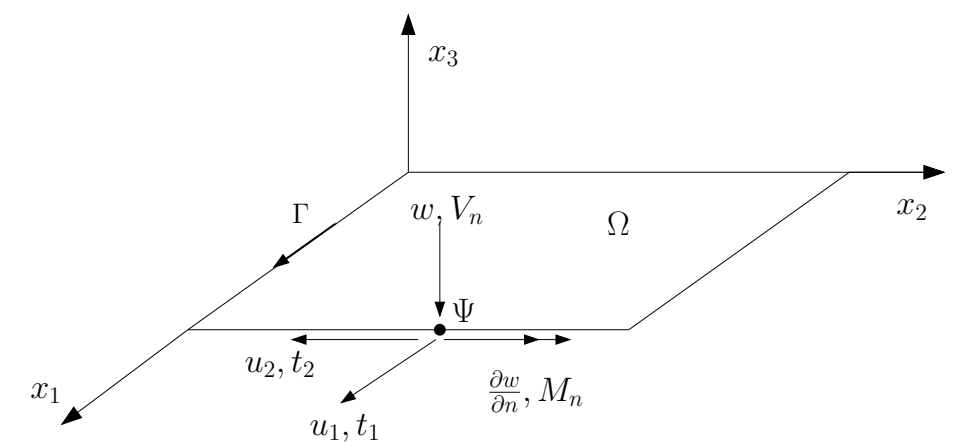

Figura 7.1: Deslocamentos e esforços em um nó genérico $\Psi$ de um macro-elemento.

$$
\mathbf{u}=\left\{\begin{array}{c}
u_{1} \\
u_{2} \\
w \\
\frac{\partial w}{\partial n}
\end{array}\right\}
$$


e o vetor t é definido como o vetor de esforços do nó $\Psi$ do macro-elemento e é dado por:

$$
\mathbf{t}=\left\{\begin{array}{c}
t_{1} \\
t_{2} \\
V_{n} \\
M_{n},
\end{array}\right\}
$$

O seguinte sistema algébrico pode ser montado para um dado ponto fonte sendo um nó do contorno, com o contorno do macro-elemento com $N C$ cantos discretizado em $N E$ elementos de contorno constantes.

$$
\begin{gathered}
\frac{1}{2}\left\{\begin{array}{c}
u_{1}^{(d)} \\
u_{2}^{(d)} \\
w^{(d)} \\
\frac{\partial w^{(d)}}{\partial n}
\end{array}\right\}+\sum_{i=1}^{N E}\left(\left[\begin{array}{cccc}
h_{11} & h_{12} & 0 & 0 \\
h_{21} & h_{22} & 0 & 0 \\
0 & 0 & h_{11}^{w} & h_{12}^{w} \\
0 & 0 & h_{21}^{w} & h_{22}^{w}
\end{array}\right]\left\{\begin{array}{c}
u_{1} \\
u_{2} \\
w \\
\frac{\partial w}{\partial n}
\end{array}\right\}\right)= \\
\sum_{i=1}^{N E}\left(\left[\begin{array}{cccc}
g_{11} & g_{12} & 0 & 0 \\
g_{21} & g_{22} & 0 & 0 \\
0 & 0 & g_{11}^{w} & g_{12}^{w} \\
0 & 0 & g_{21}^{w} & g_{22}^{w}
\end{array}\right]\left\{\begin{array}{c}
t_{1} \\
t_{2} \\
V_{n} \\
M_{n}
\end{array}\right\}\right)+\sum_{i=1}^{N C}\left(\left\{\begin{array}{c}
0 \\
0 \\
C_{1}^{(i, d)} \\
C_{2}^{(i, d)}
\end{array}\right\} w_{c}^{(i)}\right)+\left\{\begin{array}{c}
P_{1} \\
P_{2} \\
P_{3} \\
P_{4}
\end{array}\right\} .
\end{gathered}
$$

Caso o ponto fonte seja um ponto interno, a equação 7.3 é escrita como:

$$
\begin{gathered}
\left\{\begin{array}{c}
u_{1}^{(d)} \\
u_{2}^{(d)} \\
w^{(d)}
\end{array}\right\}+\sum_{i=1}^{N E}\left(\left[\begin{array}{cccc}
h_{11} & h_{12} & 0 & 0 \\
h_{21} & h_{22} & 0 & 0 \\
0 & 0 & h_{11}^{w} & h_{12}^{w}
\end{array}\right]\left\{\begin{array}{c}
u_{1} \\
u_{2} \\
w \\
\frac{\partial w}{\partial n}
\end{array}\right\}\right)= \\
\sum_{i=1}^{N E}\left(\left[\begin{array}{cccc}
g_{11} & g_{12} & 0 & 0 \\
g_{21} & g_{22} & 0 & 0 \\
0 & 0 & g_{11}^{w} & g_{12}^{w}
\end{array}\right]\left\{\begin{array}{c}
t_{1} \\
t_{2} \\
V_{n} \\
M_{n}
\end{array}\right\}\right)+\sum_{i=1}^{N C}\left(\left\{\begin{array}{c}
0 \\
0 \\
C_{1}^{(i, d)}
\end{array}\right\} w_{c}^{(i)}\right)+\left\{\begin{array}{c}
P_{1} \\
P_{2} \\
P_{3}
\end{array}\right\} .
\end{gathered}
$$

Caso o ponto fonte seja um canto, a equação (7.3) é escrita como:

$$
c w^{(d)}+\sum_{i=1}^{N E}\left(\left[\begin{array}{ll}
h_{11}^{w} & h_{12}^{w}
\end{array}\right]\left\{\begin{array}{c}
w \\
\frac{\partial w}{\partial n}
\end{array}\right\}\right)=
$$




$$
\sum_{i=1}^{N E}\left(\left[\begin{array}{ll}
g_{11}^{w} & g_{12}^{w}
\end{array}\right]\left\{\begin{array}{c}
V_{n} \\
M_{n}
\end{array}\right\}\right)+\sum_{i=1}^{N C}\left(C_{1}^{(i, d)} w_{c}^{(i)}\right)+P_{3}
$$

Os termos não nulos da primeira e segunda linha das matrizes (7.3) e (7.4) são referentes às equações integrais do estado plano, enquanto que os termos não nulos da terceira e quarta linha da equação (7.3) e terceira linha da equação (7.4) referem às equações integrais de flexão de placas representadas pelo índice $w$. A equação (7.5) é referente ao deslocamento do canto na direção transversal ao macro-elemento. Nas matrizes, observa-se que, os estados plano de tensão e o regime de flexão de placa estão desacoplados e, as equações finais encontradas são independente. O acoplamento destas equações se dá quando dois macro-elementos pertencentes a planos diferentes estão acoplados a uma mesma interface.

\subsubsection{Montagem do sistema de equações}

Após as integrações das equações integrais dos problemas da elasticidade plana e de flexão de placas finas sobre os $N E$ elementos considerando como pontos fontes os $N E$ nós do contorno do macro-elemento, os NPI pontos internos e os $N C$ cantos, pode-se escrever genericamente o seguinte sistema algébrico de $4 N E+3 N P I+N C$ equações:

$$
\mathbf{H} \mathbf{U}=\mathbf{G} \mathbf{T}+\mathbf{b}
$$

onde $\mathbf{H}$ e $\mathbf{G}$ são matrizes de influência do MEC de dimensão $(4 N E+3 N P I+N C) \times$ $(4 N E+3 N P I+N C)$, o vetor $\mathbf{U}$ contém deslocamentos e rotações dos nós do contorno e os deslocamentos dos pontos internos e cantos (lembrando que o deslocamento do canto é só na direção transversal ao macro-elemento). Os vetores $\mathbf{T}$ contém reações (forças e momento) nos nós do contorno. O vetor $\mathbf{b}$ é devido à carga transversal distribuída aplicada no domínio do macro-elemento.

\subsection{Associação espacial de macro-elementos}

Neste seção, é desenvolvida a formulação para associação espacial de macro-elementos através da técnica de sub-regiões do MEC.

\subsubsection{Sistema de coordenadas}

Uma das principais características das equações integrais de flexão de placas e estado plano de tensão é que o deslocamento de um ponto é descrito em função de sua posição 
relativa aos demais pontos do problema. Portanto, as relações entre as forças e os deslocamentos no contorno serão preservadas independente do sistema de coordenadas usado para a montagem das matrizes do problema. Assim, cada macro-elemento pode possuir seu próprio sistema de coordenadas, no qual suas equações integrais são escritas.

Quando se tem a associação de macro-elementos, o sistema de coordenadas pode não ser o mesmo para cada macro-elemento. Como se deseja compatibilizar forças e deslocamentos de vários macro-elementos, resolvendo-os simultaneamente, é necessário escrever essas incógnitas em um mesmo sistema global de coordenadas. Assim, tornase necessário escrever equações de transformação de coordenadas para cada macroelemento, relacionando-se os sistemas local e global de coordenadas.

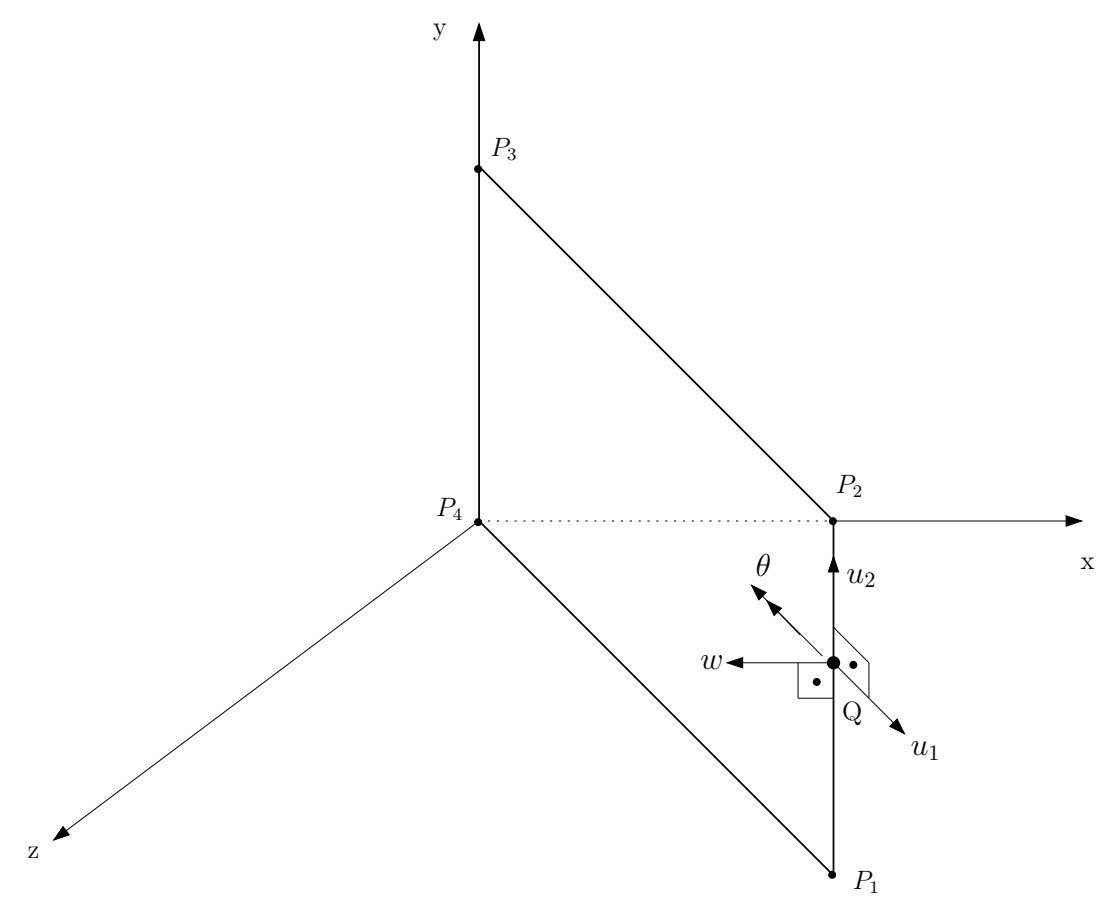

Figura 7.2: Sistema de Coordenadas.

Considere o sistema de coordenadas da Figura 7.2. Seja $U^{\prime}$ o vetor de deslocamentos no ponto $Q$ expresso no sistema local de coordenadas da seguinte forma:

$$
\mathbf{U}^{\prime}=\left\{\begin{array}{c}
u_{1}^{\prime} \\
u_{2}^{\prime} \\
w^{\prime} \\
\theta^{\prime}
\end{array}\right\}
$$

onde $u_{1}^{\prime}, u_{2}^{\prime}$ e $w^{\prime}$ são os deslocamentos nas direções dos vetores $\mathbf{n}, \mathbf{l}$ e $\mathbf{m}$. A rotação $\theta$ é dada por $\theta=\frac{\partial w}{\partial n}$.

O vetor $\mathbf{n}$ é dado por: 


$$
\mathbf{n}=\frac{P_{2}-P_{1}}{\left\|P_{2}-P_{1}\right\|}=\left\{n_{1}, n_{2}, n_{3}\right\}
$$

onde $P_{2}$ e $P_{1}$ são as coordenadas dos pontos que definem a primeira aresta do macroelemento. Convém lembrar que neste trabalho todas as arestas são retilíneas.

Um vetor $\mathbf{l}^{\prime}$ (que pode ou não coincidir com o vetor l) é calculado como:

$$
\mathbf{l}^{\prime}=\frac{P_{3}-P_{2}}{\left\|P_{3}-P_{2}\right\|}=\left\{l_{1}^{\prime}, l_{2}^{\prime}, l_{3}^{\prime}\right\}
$$

onde $P_{3}$ é o terceiro ponto que define o macro elemento e, juntamente com $P_{2}$, define a segunda aresta do macro elemento. Caso a segunda aresta não seja ortogonal a primeira aresta, o vetor $\mathbf{l}^{\prime}$ não irá coincidir com o vetor $\mathbf{l}$ que será calculado a seguir.

$\mathrm{O}$ vetor $\mathbf{m}$, perpendicular ao macro-elemento, é dado por:

$$
\mathbf{m}=\mathbf{n} \times \mathbf{l} \boldsymbol{\prime}=\left\{m_{1}, m_{2}, m_{3}\right\},
$$

O vetor 1 é dado por:

$$
\mathbf{l}=\mathbf{m} \times \mathbf{n}=\left\{l_{1}, l_{2}, l_{3}\right\},
$$

Passando ao sistema global de coordenadas, o vetor de deslocamentos é expresso como:

$$
\mathbf{U}=\left\{\begin{array}{l}
u_{1} \\
u_{2} \\
w \\
\theta
\end{array}\right\}
$$

Os deslocamentos $u_{1}, w, u_{2}$, no sitema global de coordenadas, podem ser escritos em termos dos deslocamentos $u_{1}^{\prime}, w^{\prime}, u_{2}^{\prime}$ no sistema local, como:

$$
\left\{\begin{array}{l}
u_{1} \\
u_{2} \\
w \\
\theta
\end{array}\right\}=\left[\begin{array}{cccc}
n_{1} & m_{1} & l_{1} & 0 \\
n_{2} & m_{2} & l_{2} & 0 \\
n_{3} & m_{3} & l_{3} & 0 \\
0 & 0 & 0 & \beta
\end{array}\right]\left\{\begin{array}{c}
u_{1}^{\prime} \\
u_{2}^{\prime} \\
w^{\prime} \\
\hat{\theta}
\end{array}\right\}
$$

onde $\beta$ depende da orientação dos macro-elementos que dividem a interface (veja a seção 7.3.2).

De maneira compacta:

$$
\mathbf{u}=\mathbf{T} \mathbf{u}^{\prime}
$$


onde $\mathbf{T}$ é a matriz que transforma o vetor de deslocamento $\mathbf{u}^{\prime}$ escrito em coordenadas locais em um vetor $\mathbf{u}$ expresso em coordenadas globais.

Adotando o mesmo procedimento para descrever o vetor de esforços $\mathbf{t}$ em coordenadas globais, tem-se:

$$
\left\{\begin{array}{l}
t_{1} \\
t_{2} \\
V_{n} \\
M_{n}
\end{array}\right\}=\left[\begin{array}{cccc}
n_{1} & m_{1} & l_{1} & 0 \\
n_{2} & m_{2} & l_{2} & 0 \\
n_{3} & m_{3} & l_{3} & 0 \\
0 & 0 & 0 & \beta
\end{array}\right]\left\{\begin{array}{c}
t_{1} \\
M_{n}^{\prime} \\
t_{2}^{\prime} \\
V_{n}^{\prime}
\end{array}\right\},
$$

\subsubsection{Compatibilização de momentos e rotações}

Na associação de macro-elementos, aparecem na interface, momentos de flexão e rotações em torno da inteface. As direções destes momentos e rotações não são alterados com a aplicação da matriz de transformação apresentada na seção anterior pois ambos são em torno da interface (aresta comum). O sentido, porém, pode-se inverter dependendo do sentido das arestas que compõe a interface. Adota-se $\beta=1$ na matriz de transformação dos dois macro elementos se os momentos locais e as rotações têm sentidos opostos. Caso contrário, adota-se $\beta=1$ no primeiro macro elemento e $\beta=-1$ no segundo. O mesmo se faz com a rotação $\frac{\partial v}{\partial n}$ (veja figura 7.3).

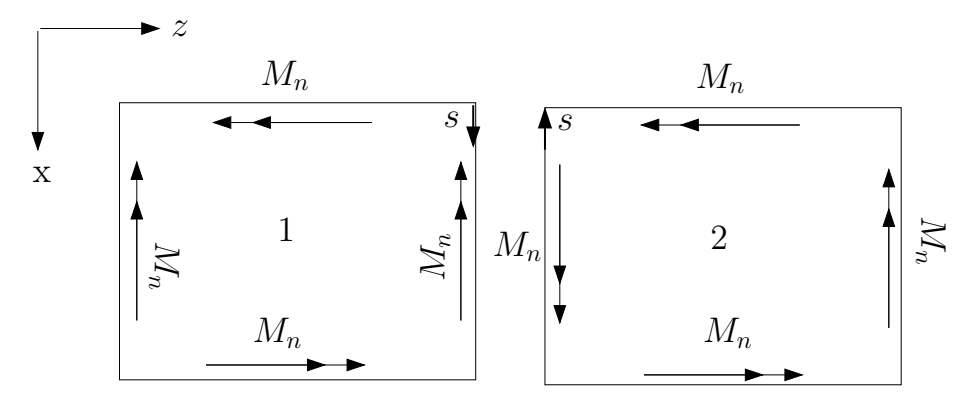

Figura 7.3: Compatibilização de momentos e rotações nos macro-elementos (neste caso $\beta=1)$.

\subsubsection{Associação de macro-elementos via método de sub-regiões do MEC}

Seja a associação plana de dois macro-elementos conforme a Figura 7.4. De acordo com o que foi desenvolvido em capítulos anteriores, o vetor de deslocamentos em um dado nó no contorno de um macro-elemento, possui 4 graus de liberdade, dois referentes ao estado plano e dois referente à flexão de placas. Sabe-se que, em nós pertencentes a 
interface dos macro-elementos existem 8 variáveis desconhecidas $\left(u_{1}, u_{2}, w, \theta, t_{1}, t_{2}, V_{n}\right.$ e $\left.M_{n}\right)$. Através da aplicação das condições de equilíbrio e continuidade de deslocamentos na interface, as variáveis desconhecidas são reduzidas a 4 (Sanches, 2002).

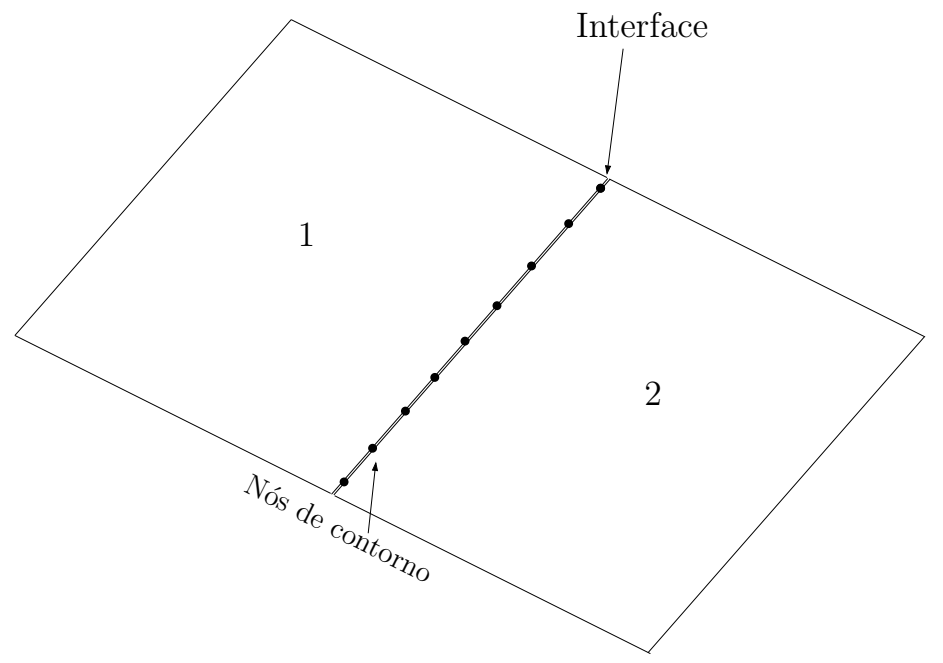

Figura 7.4: Associação de dois macro-elementos.

As condições de continuidade de deslocamento são dadas por:

$$
\begin{aligned}
& u_{i}^{1}=u_{i}^{2}, \\
& t_{i}^{1}=t_{i}^{2}, \\
& \theta_{i}^{1}=\theta_{i}^{2}
\end{aligned}
$$

Para as condições equilíbrio de forças de superfície $t_{i}$, esforço cortante $V_{n}$ e momentos, $M_{i}$ Baiz and Aliabadi (2009b); Pisa (2005):

$$
\begin{aligned}
t_{i}^{1}+t_{i}^{2}+t_{i} & =0 \\
V_{n}^{1}+V_{n}^{2}+V_{n} & =0 \\
M_{n}^{1}+M_{n}^{2}+M_{n} & =0
\end{aligned}
$$

onde os índices superiores referem-se ao número de macro-elemento.

Nas equações apresentadas em (7.17), a terceira parcela de cada equação refere-se aos esforços na aresta. Estas forças relacionam-se com os esforços da interface, ou quando o deslocamento é incógnito, a força é conhecida ou vice-versa, ou seja, não há aumento do número de incógnitas. O sistema final de equações algébricas obtido após o cálculo das integrais pode ser escrito da seguinte forma:

$$
\mathbf{H} \mathbf{U}=\mathbf{G} \mathbf{T}+\mathbf{b}
$$


onde as matrizes elementares que fazem parte das matrizes finais $\mathbf{H}$ e $\mathbf{G}$ da Equação (7.18), são montadas a partir das equações de transformação de coordenadas locais em globais mostrada na seção 7.3.1.

Usando a técnica de sub-regiões do MEC, a equação matricial correspondente ao macro-elemento 1 pode ser escrita como:

$$
\left[\begin{array}{ll}
\mathbf{H}_{11}^{1} & \mathbf{H}_{1 i}^{1} \\
\mathbf{H}_{i 1}^{1} & \mathbf{H}_{i i}^{1}
\end{array}\right]\left\{\begin{array}{c}
\mathbf{U}_{1} \\
\mathbf{U}_{A}
\end{array}\right\}=\left[\begin{array}{ll}
\mathbf{G}_{11}^{1} & \mathbf{G}_{1 i}^{1} \\
\mathbf{G}_{i 1}^{1} & \mathbf{G}_{i i}^{1}
\end{array}\right]\left\{\begin{array}{c}
\mathbf{T}_{1} \\
\mathbf{T}_{A}
\end{array}\right\}+\left\{\begin{array}{c}
\mathbf{b}_{1} \\
\mathbf{b}_{A}^{1}
\end{array}\right\}
$$

da mesma forma, para o macro-elemento 2, têm-se:

$$
\left[\begin{array}{cc}
\mathbf{H}_{11}^{2} & \mathbf{H}_{1 i}^{2} \\
\mathbf{H}_{i 1}^{2} & \mathbf{H}_{i i}^{2}
\end{array}\right]\left\{\begin{array}{c}
\mathbf{U}_{2} \\
\mathbf{U}_{A}
\end{array}\right\}=\left[\begin{array}{ll}
\mathbf{G}_{11}^{2} & \mathbf{G}_{1 i}^{2} \\
\mathbf{G}_{i 1}^{2} & \mathbf{G}_{i i}^{2}
\end{array}\right]\left\{\begin{array}{c}
\mathbf{T}_{2} \\
\mathbf{T}_{A}
\end{array}\right\}+\left\{\begin{array}{c}
\mathbf{b}_{2} \\
\mathbf{b}_{A}^{2}
\end{array}\right\}
$$

Os termos $\mathbf{H}_{\mathbf{i j}}^{\mathbf{2}}$ e $\mathbf{G}_{\mathbf{i j}}^{\mathbf{2}}$, são as matrizes de influência das formulações de flexão de placas e elasticidade plana, como abordado em Dirgantara and Aliabadi (2001). Sendo:

1. $\mathbf{U}_{1}$ e $\mathbf{U}_{2}$ os deslocamentos nodais sobre o contorno dos macro-elementos 1 e 2, respectivamente.

2. $\mathbf{T}_{\mathbf{1}}$ e $\mathbf{T}_{\mathbf{2}}$ os esforços nodais sobre o contorno dos macro-elementos 1 e 2 , respectivamente.

3. $\mathbf{b}_{\mathbf{1}} \mathbf{e} \mathbf{b}_{\mathbf{2}}$ as integrais devido às forças de corpo.

4. $\mathbf{U}_{\mathbf{A}}, \mathbf{T}_{\mathbf{A}}$ e $\mathbf{b}_{\mathbf{A}}$ os deslocamentos, esforços nodais e as integrais devido as forças de corpo na aresta da interface, respectivamente.

5. $\mathbf{H}_{\mathbf{i j}}^{\mathbf{k}}$ e $\mathbf{G}_{\mathbf{i j}}^{\mathbf{k}}$ sub-matrizes, dos efeitos $i$ em $j$ do macro elemento $k$.

Arranjando as equações de diferentes regiões em um único sistema, a forma matricial para as placas associadas fica:

$$
\left[\begin{array}{cccccc}
\mathbf{H}_{1}^{(1)} & \mathbf{H}_{12}^{(1)} & \mathbf{0} & \mathbf{0} & -\mathbf{G}_{12}^{(1)} & \mathbf{0} \\
\mathbf{0} & \mathbf{0} & \mathbf{H}_{2}^{(2)} & -\mathbf{H}_{21}^{(2)} & \mathbf{0} & -\mathbf{G}_{21}^{(2)} \\
\mathbf{0} & \mathbf{0} & \mathbf{0} & \mathbf{0} & \mathbf{E}_{12} & \mathbf{E}_{21} \\
\mathbf{0} & \mathbf{C}_{12} & \mathbf{0} & \mathbf{C}_{21} & \mathbf{0} & \mathbf{0}
\end{array}\right]\left\{\begin{array}{c}
u_{1}^{(1)} \\
u_{12}^{(1)} \\
u_{2}^{(2)} \\
u_{21}^{(2)} \\
t_{12}^{(1)} \\
t_{21}^{(2)}
\end{array}\right\}=\left[\begin{array}{cc}
\mathbf{G}_{1}^{(1)} & \mathbf{0} \\
\mathbf{0} & \mathbf{G}_{2}^{(2)}
\end{array}\right]\left\{\begin{array}{l}
t_{1}^{(1)} \\
t_{2}^{(2)}
\end{array}\right\}+\left\{\begin{array}{l}
b_{1}^{(1)} \\
b_{2}^{(2)}
\end{array}\right\}
$$

na qual o sobre escrito $(i)$ indica a sub região do problema, os sub escritos 1 e 2 indicam os elementos das matrizes e vetores pertencentes somente àquela região. Os sub escritos 
cruzados 12 e 21 indicam elementos das matrizes e vetores pertencentes à região 1 e na interface com a 2 e vice versa. As matrizes $\mathbf{E}_{12}$ e $\mathbf{E}_{21}$ são devido às condições de equilíbrio, $\mathbf{C}_{12}$ e $\mathbf{C}_{21}$ são aquelas devido às condições de compatibilidade, dadas por:

$$
\begin{gathered}
\mathbf{E}_{12}=\mathbf{E}_{21}=\mathbf{C}_{12}=\mathbf{T} \\
\mathbf{C}_{21}=-\mathbf{T}
\end{gathered}
$$

\subsection{Aspectos computacionais}

As rotinas usadas para gerar os resultados desta tese, partem de códigos já implementados Hoefel et al. (2005) e foram alterados de maneira a acomodar esta nova versão da formulação para associação de placas finas e de elasticidade plana. Todos os programas foram implementadas em código do software Matlab. A maior parte dos procedimentos computacionais já foram descritos nesta tese de maneira implícita.

Os programas desenvolvidos seguem a seguinte rotina básica:

1. Entrada de dados;

2. Geração de visualização da geometria e das condições de contorno do problema;

3. Verificação de interfaces e sub regiões;

4. Geração de Malha para o problema;

5. Montagem das matrizes de influência para cada uma das sub regiões com resolução das integrais;

6. Posicionamento destas matrizes no sistema global;

7. Imposição de condições de compatibilidade e equilíbrio;

8. Solução do sistema equivalente por integração analítica;

9. Transformação dos deslocamentos para o sistema global;

10. Geração de tabelas e gráficos contendo as resposta para visualização e avaliação. 


\section{Capítulo 8}

\section{Resultados Numéricos para Problemas Estáticos}

\subsection{Introdução}

Nesta seção serão apresentados exemplos numéricos usando elementos constantes com objetivo de validar a formulação de sub-regiões para associação de placas no plano e espaço.

Quando o problema é de elasticidade plana, ele é chamado de chapa. Quando se trata de placas de Kirchhoff, o problema é chamado de placa. Os problemas apresentados aqui foram, em sua maioria, analisados por outros autores usando uma formulação de placas deformáveis pelo cisalhamento.

As respostas serão comparados com soluções analíticas, sempre que estas estiverem disponíveis, e com os resultados obtidos utilizando as formulações de elasticidade plana ou de placas de Kirchhoff sem sub-regiões. Para aqueles problemas que não possuem solução analítica disponível, uma resposta numérica obtida com o MEF com o auxílio do Ansys foi obtida. A análise foi feita utilizando o elemento SHELL181 que é adequado para a análise de estruturas formadas por cascas ou placas finas a moderadamente espessas. Este elemento é quadrilateral de 4 nós e possui 24 graus de liberdade, 6 para cada um dos nós. Três deslocamentos e três rotações em relação aos eixos $x, y$ e $z$. Os erros relativos apresentados neste capítulo e no seguinte foram calculados sempre que havia uma solução analítica disponível e foram obtidos a partir da equação (8.1):

$$
\operatorname{Erro}(\%)=\operatorname{abs}\left(\frac{R^{\text {num }}-R^{a n}}{R^{a n}}\right) \times 100
$$

onde $R^{\text {num }}$ é a resposta numérica e $R^{\text {an }}$ a analítica. 


\subsection{Elasticidade Plana}

\subsubsection{Chapa tracionada}

Neste exemplo, considera-se uma hipotética chapa quadrada monoengastada submetida a uma força axial $q=1 \mathrm{~N} / \mathrm{m}^{2}$, conforme Figura 8.1, que servirá como teste para a avaliação de deslocamento no plano. O modelo foi discretizado utilizando elementos de contorno constantes. As propriedades geométrica são: $L=1 \mathrm{~m}$ e espessura $t=1 \mathrm{~m}$. As propriedades do material são: módulo de elasticidade $E=1 \mathrm{~Pa}$, e razão de Poisson $\nu=0.0$. Os resultados obtidos para o ponto $A$ são comparados com a solução analítica.

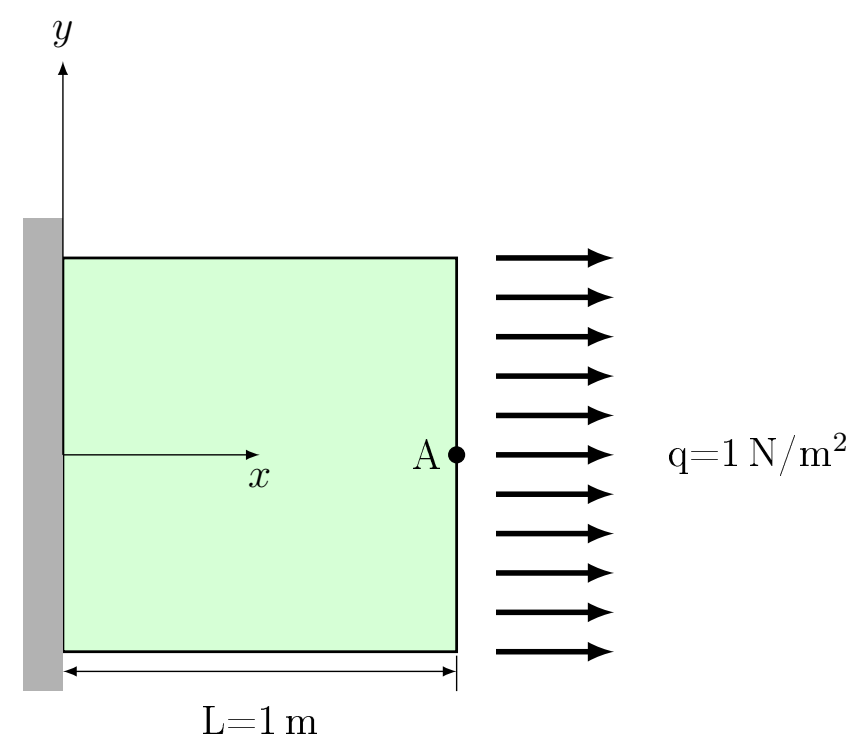

Figura 8.1: Ilustração da placa submetida a força axial.

Sendo assim, foram simulados alguns testes de convergência de malhas, nos quais foram variados os números de elementos da malha. A Tabela 8.1 analisa o deslocamento na direção $x$ no ponto $A(x=1, y=0)$ para 1 e 2 subregiões. O número de elementos é o número total, ou seja, no caso de duas sub-regiões é a soma dos elementos da duas sub-regiões. O valor analítico do deslocamento é:

$$
w=1,00 \mathrm{~m}
$$

Conforme pode ser observado na Tabela 8.1, o resultado tende a solução analítica tanto para uma como para duas sub-regiões. Porém, com duas sub-regiões a convergência é ligeiramente melhor, necessitando de menos elementos para um erro de mesma ordem. 
Tabela 8.1: Resultados obtidos para placa - força axial.

\begin{tabular}{cccccc}
\hline $\begin{array}{c}\text { Números de } \\
\text { elementos }\end{array}$ & $\begin{array}{c}\text { Deslocamento } \\
\text { 1 sub-região }\end{array}$ & $\begin{array}{c}\text { Erro } \\
(\%)\end{array}$ & $\begin{array}{c}\text { Números de } \\
\text { elementos }\end{array}$ & $\begin{array}{c}\text { Deslocamento } \\
\text { 2 sub-regiões }\end{array}$ & $\begin{array}{c}\text { Erro } \\
(\%)\end{array}$ \\
\hline 8 & 0,9755 & 2,45 & 24 & 0,9992 & 0,08 \\
20 & 0,9959 & 0,41 & 60 & 0,9995 & 0,05 \\
40 & 0,9983 & 0,17 & 96 & 0,9997 & 0,03 \\
80 & 0,9993 & 0,07 & 144 & 0,9998 & 0,02 \\
160 & 0,9997 & 0,03 & 216 & 0,9999 & 0,01 \\
400 & 0,9999 & 0,01 & 612 & 1,0000 & 0 \\
800 & 1,0000 & 0 & - & - & - \\
\hline
\end{tabular}




\subsubsection{Placa apoiada}

A Figura 9.1 mostra o problema físico considerado: uma placa simplesmente apoiada em quatro lados, submetida a carregamento constante distribuído uniformemente por toda a superfície. As propriedades geométricas são: $L=1 \mathrm{~m}$ e espessura $t=$ 0,008 m. As propriedades do material são: módulo de elasticidade $E=210 \mathrm{GPa}$, razão de Poisson $\nu=0,3$ e carregamento $q=100 \mathrm{~N} / \mathrm{m}^{2}$.

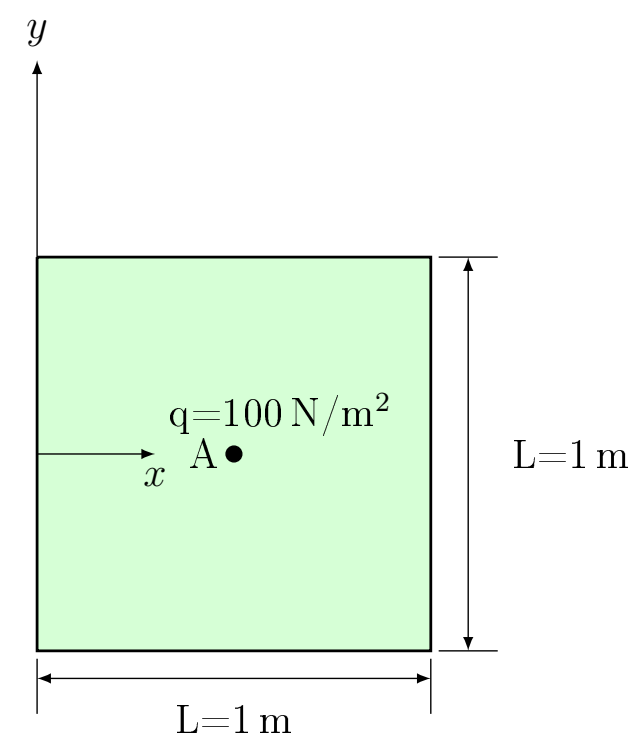

Figura 8.2: Ilustração da placa apoiada.

A solução analítica para este problema obtido no centro da placa é calculada segundo Timoshenko e Woinowsky-Krieger (1959) Timoshenko and Woinowski-Krieger (1959). Esta solução é dada por:

$$
w=\frac{w^{\prime} q}{D}
$$

onde $D$ é a rigidez à flexão da placa e $w^{\prime}$ é a deflexão normalizada.

Aplicando as propriedades do problema, encontra-se os resultados mostrados na Tabela 8.2 para o ponto $A(x=0,5, y=0)$. Uma vez que $w^{\prime}=0,0041$, o valor do deslocamento calculado segundo a equação (8.2) é:

$$
w=4,1641.10^{-5} \mathrm{~m}
$$

Os deslocamentos transversais para a placa deformada são observados na Tabela 8.2 .

Pode-se notar que com 2 sub-regiões é necessário um número muito maior de elementos para se obter o mesmo erro obtido com uma sub-região. Este comportamento 

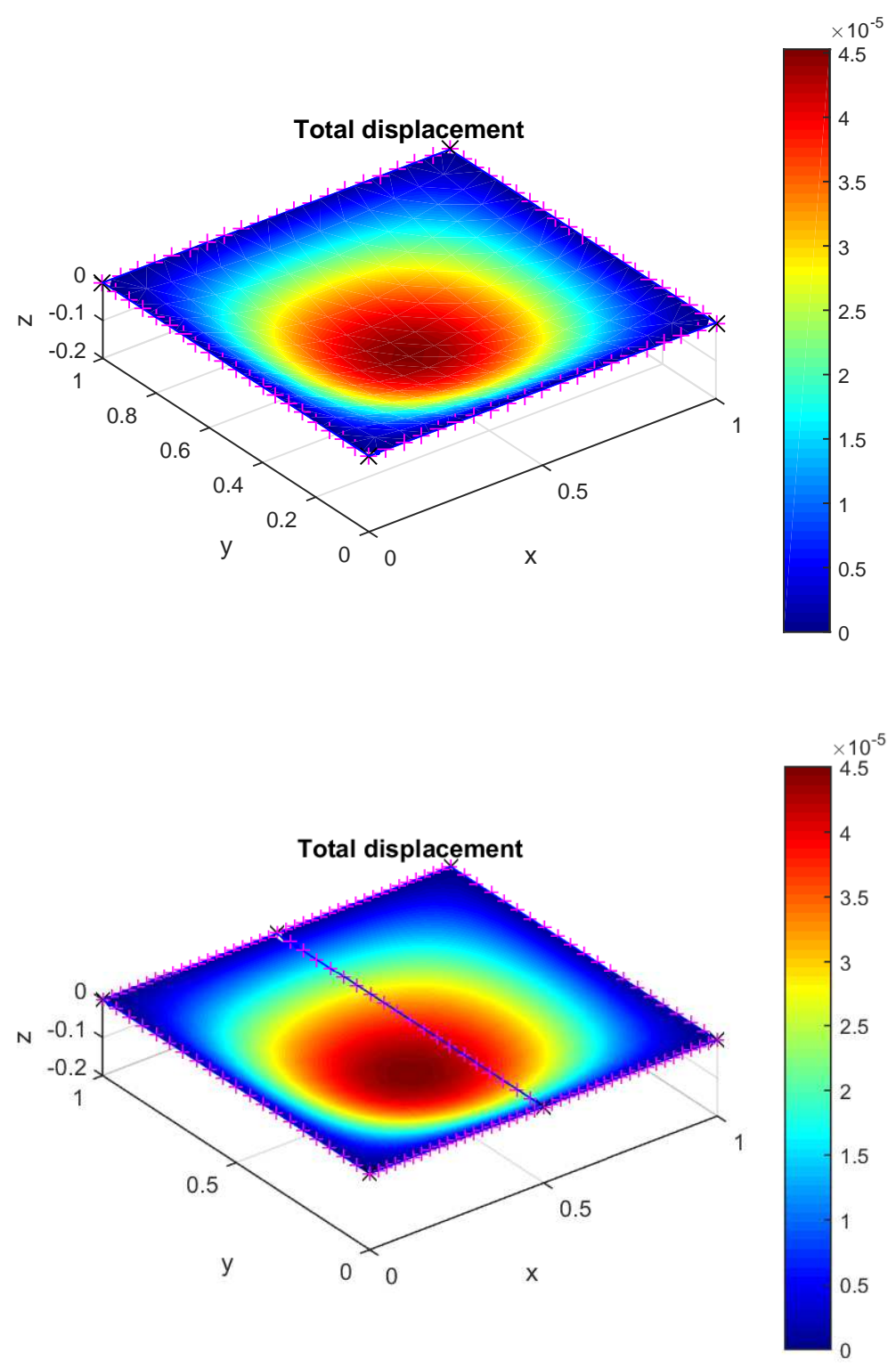

Figura 8.3: (a) Deslocamento na direção $z$ para a placa apoiada usando 1 sub-região. (b) Deslocamento na direção $z$ para a placa apoiada usando 2 sub-regiões.

foi o oposto do problema anterior. Em ambos os casos, a convergência foi mais lenta que no caso anterior. 
Tabela 8.2: Resultados obtidos para placa apoiada.

\begin{tabular}{cccccc}
\hline $\begin{array}{c}\text { Números de } \\
\text { elementos }\end{array}$ & $\begin{array}{c}\text { Deslocamento } \\
\text { 1 sub-região }\end{array}$ & $\begin{array}{c}\text { Erro } \\
(\%)\end{array}$ & $\begin{array}{c}\text { Número de } \\
\text { elementos }\end{array}$ & $\begin{array}{c}\text { Deslocamento } \\
\text { 2 sub-regiões }\end{array}$ & $\begin{array}{c}\text { Erro } \\
(\%)\end{array}$ \\
\hline 12 & $5,102010^{-05}$ & 22,523 & 60 & $4,893410^{-05}$ & 17,514 \\
20 & $4,675210^{-05}$ & 12,274 & 120 & $4,751110^{-05}$ & 14,096 \\
40 & $4,542510^{-05}$ & 9,087 & 300 & $4,627610^{-05}$ & 11,130 \\
60 & $4,529410^{-05}$ & 8,772 & 840 & $4,521810^{-05}$ & 8,590 \\
80 & $4,527610^{-05}$ & 8,729 & 1200 & $4,494910^{-05}$ & 7,944 \\
\hline
\end{tabular}




\subsubsection{Placa em balanço}

A Figura 8.4, mostra o problema físico considerado: uma placa isotrópica e quadrada submetida a um carregamento constante distribuído uniformemente ao longo de sua superfície. As propriedades geométricas são: $L=1 \mathrm{~m}$ e espessura $t=0,008 \mathrm{~m}$. As propriedades do material são: módulo de elasticidade $E=210 \mathrm{GPa}$, razão de Poisson $\nu=0,0$ e carregamento $q=100 \mathrm{~N} / \mathrm{m}^{2}$.
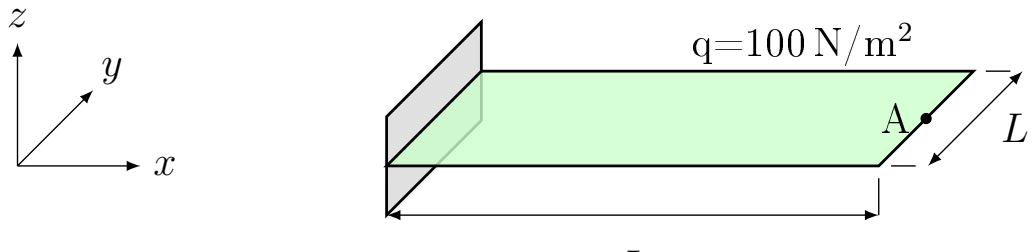

$L$

Figura 8.4: Ilustração da placa em balanço.

A verificação deste problema é desenvolvido pela comparação entre os valores de deslocamentos obtidos com a formulação de elasticidade plana usando sub-regiões e os valores calculados analiticamente, dados por:

$$
w=\frac{q L}{24 E I}\left[6 L^{2} x^{2}-4 L x^{3}+x^{4}\right]
$$

Na extremidade da placa $(x=L=1 \mathrm{~m})$, tem-se:

$$
w=0,00139 \mathrm{~m}
$$

O mapa de cor da placa sob a ação do carregamento distribuído pode ser observado na Figura 8.5. A Tabela 8.3 mostra os deslocamentos obtidos com uma e duas subregiões comparadas com a solução analítica dada pela Equação 8.4, para o ponto $A(x=$ $1, y=0,5)$.

Tabela 8.3: Resultados obtidos para placa em balanço.

\begin{tabular}{cccccc}
\hline $\begin{array}{c}\text { Números de } \\
\text { elementos }\end{array}$ & $\begin{array}{c}\text { Deslocamento } \\
\text { 1 sub-região }\end{array}$ & $\begin{array}{c}\text { Erro } \\
(\%)\end{array}$ & $\begin{array}{c}\text { Números de } \\
\text { elementos }\end{array}$ & $\begin{array}{c}\text { Deslocamento } \\
2 \text { sub-regiões }\end{array}$ & $\begin{array}{c}\text { Erro } \\
(\%)\end{array}$ \\
\hline 4 & 0,0012 & 13,669 & 84 & 0,0012 & 13,669 \\
8 & 0,0013 & 6,474 & 240 & 0,0013 & 6,474 \\
12 & 0,0014 & 0 & 288 & 0,0014 & 0 \\
\hline
\end{tabular}

Conforme observado na Tabela 8.3, como no caso anterior, a convergência com o uso de 2 sub-regiões é lenta. Para se obter um erro de mesma magnitude, quando se 

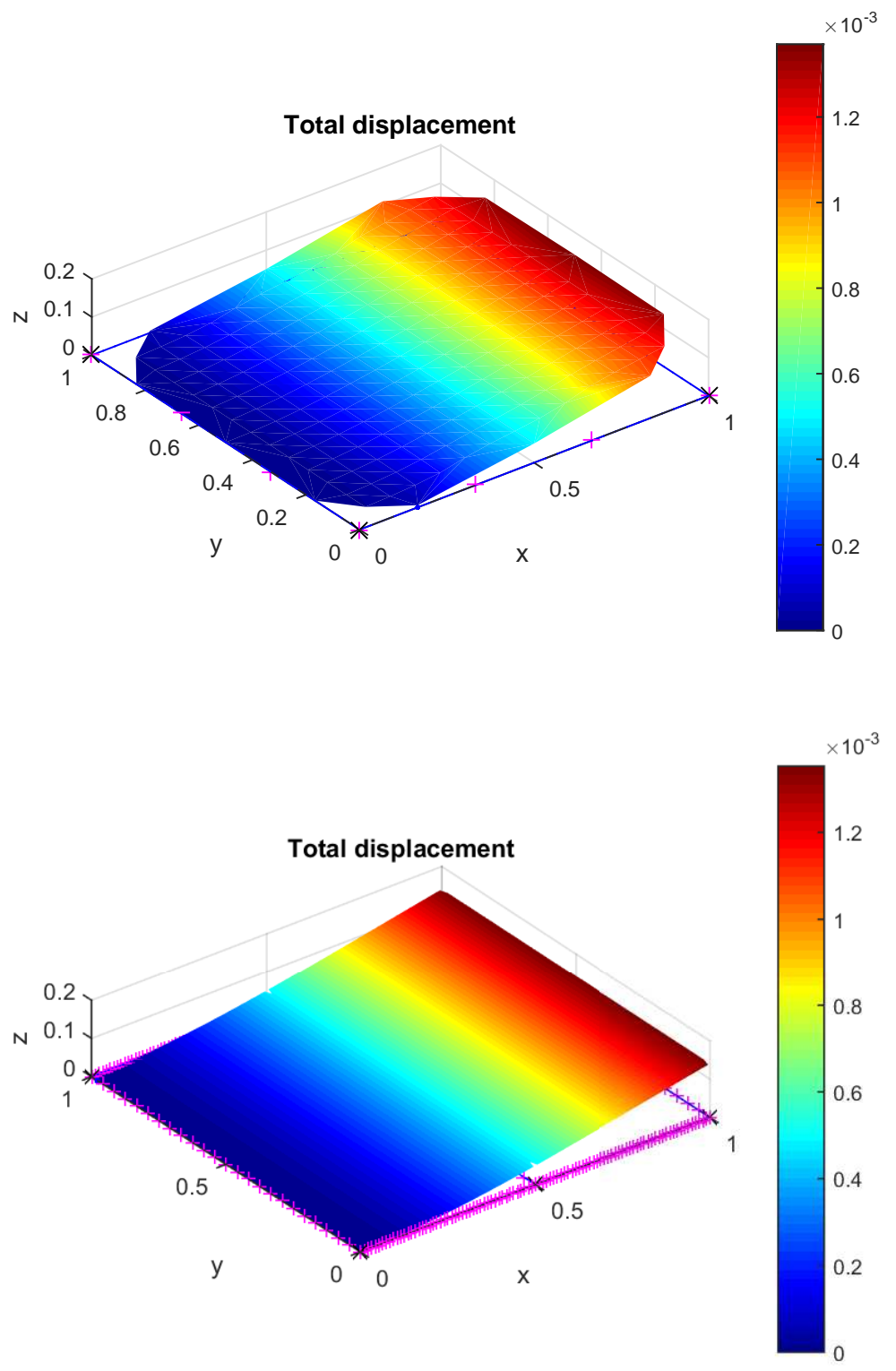

Figura 8.5: (a) Deslocamento na direção z para a placa em balanço usando 1 sub-região. (b) Deslocamento na direção $z$ para a placa em balanço usando 2 sub-regiões.

usa duas sub-regiões é necessário um número expressivamente maior de elementos que no problema modelado com uma só região. 


\subsubsection{Placa engastada}

A Figura 8.6 mostra o problema físico considerado: uma placa engastada nos quatro lados, submetida a carregamento constante distribuído uniformemente por toda a superfície. As propriedades geométricas são: $L=1 \mathrm{~m}$ e espessura $t=0,008 \mathrm{~m}$. As propriedades do material são: módulo de elasticidade $E=210 \mathrm{GPa}$, razão de Poisson $\nu=0,3$ e carregamento $q=100 \mathrm{~N} / \mathrm{m}^{2}$.

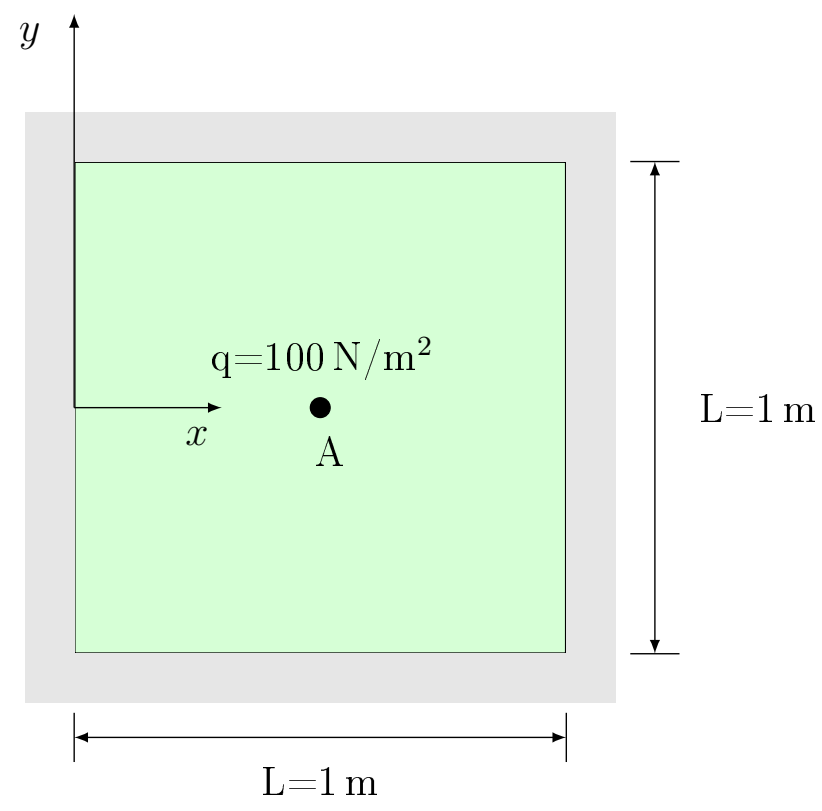

Figura 8.6: Ilustração da placa engastada.

Os gráficos da Figura 8.7 compara os deslocamentos obtidos no centro da placa na direção $z$ através do MEC com os resultados obtidos pelo Ansys, Figura 8.8.

No centro da placa, a solução analítica segundo Timoshenko and Woinowski-Krieger (1959), é dada por:

$$
w=\frac{0,00126 q L^{4}}{D}=1,4063 \cdot 10^{-5} \mathrm{~m}
$$

Aplicando as propriedades do problema, é possível encontrar os seguintes resultados para o deslocamento ponto $A(x=0,5, y=0,5)$, e comparar com a solução analítica dada pela equação (8.5). A Tabela 8.4 mostra os deslocamentos obtidos com uma e duas sub-regiões.

Mais uma vez, como nos demais casos anteriores de flexão, a convergência com o uso de 2 sub-regiões é mais lenta que com uma sub-região. Este comportamente não ocorreu, entretanto no primeiro problema deste capítulo, ou seja, no problema de elasticidade plana. 

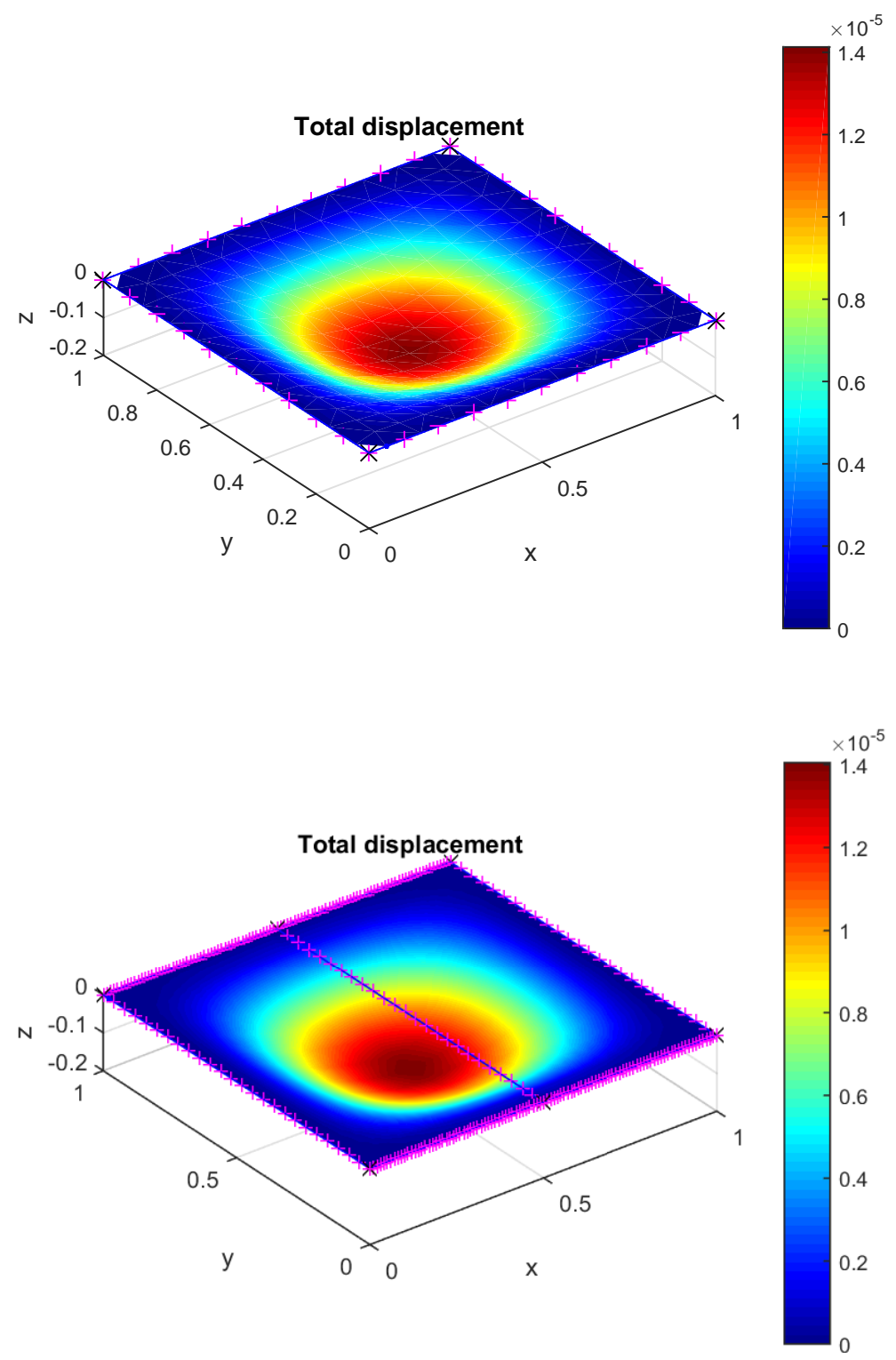

Figura 8.7: (a) Deslocamento na direção $z$ para a placa engastada usando 1 sub-região. (b) Deslocamento na direção $z$ para a placa engastada usando 2 sub-regiões. 
Tabela 8.4: Resultados obtidos para placa em balanço.

\begin{tabular}{cccccc}
\hline $\begin{array}{c}\text { Números de } \\
\text { elementos }\end{array}$ & $\begin{array}{c}\text { Deslocamento } \\
\text { 1 sub-região }\end{array}$ & $\begin{array}{c}\text { Erro } \\
(\%)\end{array}$ & $\begin{array}{c}\text { Números de } \\
\text { elementos }\end{array}$ & $\begin{array}{c}\text { Deslocamento } \\
2 \text { sub-regiões }\end{array}$ & $\begin{array}{c}\text { Erro } \\
(\%)\end{array}$ \\
\hline 4 & $1,324310^{-05}$ & 5,8309 & 36 & $1,202810^{-05}$ & 14,4706 \\
12 & $1,452210^{-05}$ & 3,2639 & 60 & $1,300010^{-05}$ & 7,5588 \\
20 & $1,416710^{-05}$ & 0,7395 & 84 & $1,341210^{-05}$ & 4,6292 \\
28 & $1,413710^{-05}$ & 0,5262 & 108 & $1,359910^{-05}$ & 3,2994 \\
36 & $1,412910^{-05}$ & 0,4693 & 180 & $1,381410^{-05}$ & 1,7706 \\
48 & $1,412510^{-05}$ & 0,4409 & 612 & $1,397110^{-05}$ & 0,6542 \\
\hline
\end{tabular}

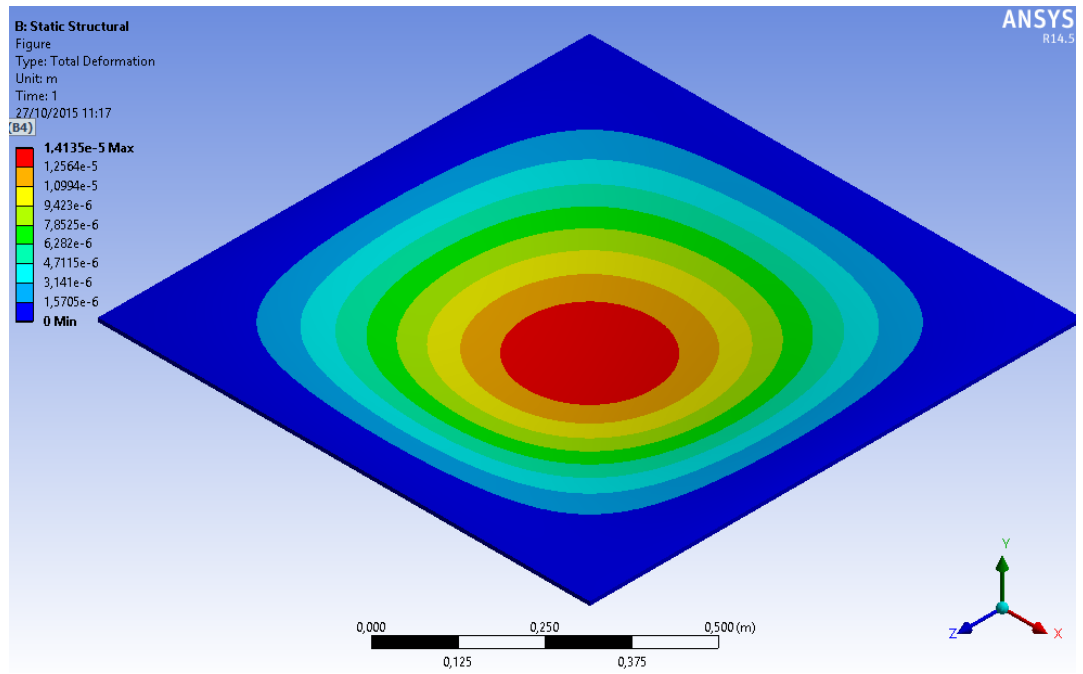

Figura 8.8: Resultados obtidos usando o MEF através do ANSYS. 


\subsection{Associação em $L$}

Este modelo é composto por duas placas associadas de maneira a formar uma estrutura em L. Este problema foi resolvido em Costa (2015) e Dirgantara and Aliabadi (2001), no qual pode ser encontrada a sua solução analítica. Na formulação proposta por Costa (2015), é usada uma formulação de placas espessas (deformáveis por cisalhamento) com a introdução do grau de liberdade de rotação na forulação de elasticidade plana. O tipo de elemento usado por Costa (2015) foi o quadrático descontínuo.

O modelo foi discretizado usando duas sub regiões. As propriedades e dimensões são dadas na Figura 8.9, onde $L_{1}=1 \mathrm{~m}, L_{2}=1 \mathrm{~m}, L_{3}=2 \mathrm{~m}, t_{1}=0,1 \mathrm{~m}$ e $t_{2}=0,1 \mathrm{~m}$. A carga é distribuída ao longo da aresta superior, orientada na direção $z$ com valor $q=1 \mathrm{~N} / \mathrm{m}$. As propriedades mecânicas são $E=100 \mathrm{kPa}$ and $\nu=0.0$.

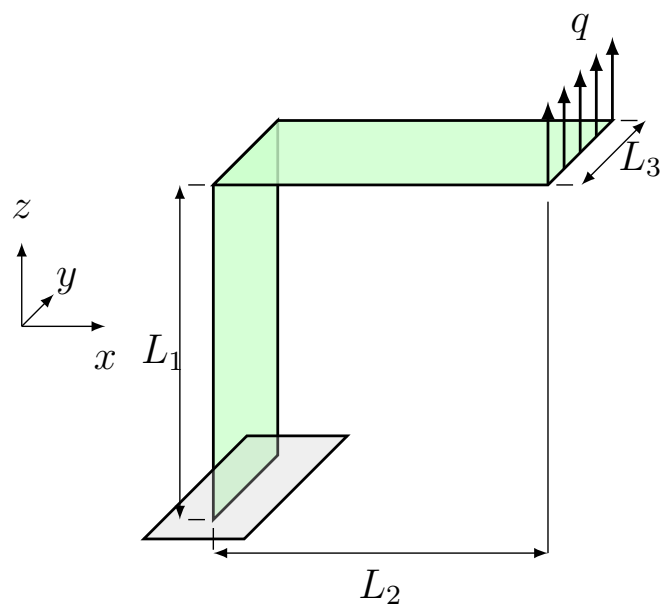

Figura 8.9: Dimensões e condições de contorno para a estrutura em $L$ (Dirgantara and Aliabadi, 2001)

Tabela 8.5: Soluções analíticas e numéricas do deslocamento na direção $z$ calculado na ponta da placa.

\begin{tabular}{cc}
\hline Método de Solução & Deslocamento na direção $(z)$ \\
\hline Costa (2015) & 0,1610 \\
Solução de Euler-Bernouli & 0,1600 \\
Solução de Timoshenko & 0,1604 \\
\hline
\end{tabular}

A distribuição dos deslocamentos pode ser visualizada na Figura 8.10 (a), com 160 elementos de contorno. A Tabela 8.5 apresenta os resultados para os deslocamentos na direção $z$ citados em Dirgantara and Aliabadi (2001) para um ponto na extremidade 
Tabela 8.6: Resultados obtidos para os deslocamentos da estrutura em $L$.

\begin{tabular}{ccccc}
\hline $\begin{array}{c}\text { Números de } \\
\text { elementos }\end{array}$ & $\begin{array}{c}\text { Deslocamento } \\
(z)\end{array}$ & $\begin{array}{c}\text { Erro (\%) } \\
\text { Costa (2015) }\end{array}$ & $\begin{array}{c}\text { Erro (\%) } \\
\text { Euler-Bernouli }\end{array}$ & $\begin{array}{c}\text { Erro (\%) } \\
\text { Timoshenko }\end{array}$ \\
\hline 40 & 0,1523 & 5,4037 & 4,8125 & 5,0499 \\
80 & 0,1540 & 4,3478 & 3,7500 & 3,9900 \\
160 & 0,1557 & 3,2919 & 2,6875 & 2,9302 \\
400 & 0,1575 & 2,1739 & 1,5625 & 1,8080 \\
800 & 0,1583 & 1,6770 & 1,0625 & 1,3092 \\
\hline
\end{tabular}

da placa superior $\left(x=L_{2}\right)$, na mesma aresta que recebe o carregamento. Aplicando as propriedades do problema, é possível encontrar os resultados mostrados na Tabela 8.6. Conforme observado nesta tabela, o resultado converge para a solução analítica com o refinamento da malha. 

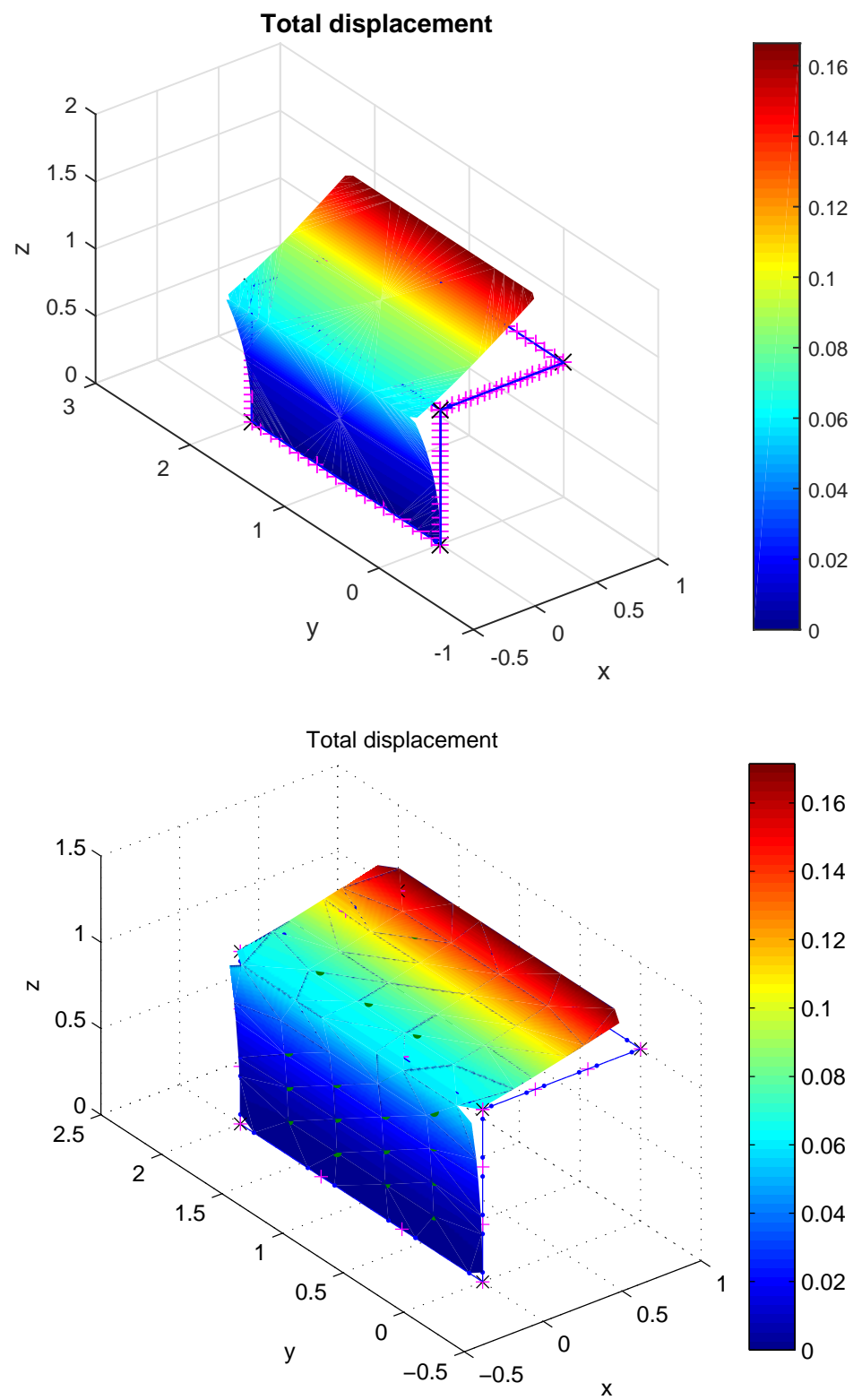

Figura 8.10: (a) Distribuição do deslocamento total para a estrutura em $L$ obtidos com a formulação do MEC de placa fina. (b) Distribuição do deslocamento total para a estrutura em $L$ obtidos com a formulação do MEC para placa espessa. 
Tabela 8.7: Soluções analíticas do deslocamento para três diferentes ângulos calculados para um ponto na extremidade carregada da placa superior.

\begin{tabular}{cccc}
\hline Deslocamento, direção $x_{3}$ & $\theta=91^{\circ}$ & $\theta=95^{\circ}$ & $\theta=120^{\circ}$ \\
\hline Costa (2015) & 0,16136 & 0,16493 & 0,16483 \\
Solução de Euler-Bernouli & 0,16102 & 0,16460 & 0,16455 \\
Solução de Timoshenko & 0,16142 & 0,16497 & 0,16476 \\
\hline
\end{tabular}

\subsubsection{Associação em $L$ em ângulos não-retos}

Este exemplo é uma variação do problema anterior, com a diferença que o ângulo de junção entre as placas pode ter valores superiores a $90^{\circ}$. Estes exemplos são utilizados para demonstrar a capacidade da formulação em modelar estruturas de placas associadas por um ângulo arbitrário. O modelo foi discretizado usando duas sub regiões. As propriedades e dimensões são mostradas na Figura 8.11, na qual $L_{1}=1 \mathrm{~m}, L_{2}=1 \mathrm{~m}$, $L_{3}=2 \mathrm{~m}, t_{1}=0,1 \mathrm{~m}$ e $t_{2}=0,1 \mathrm{~m}$. A carga distribuída na borda superior tem valor $q=1 \mathrm{~N} / \mathrm{m}$. As propriedades mecânicas são $E=100 \mathrm{kPa}$ e $\nu=0.0$.

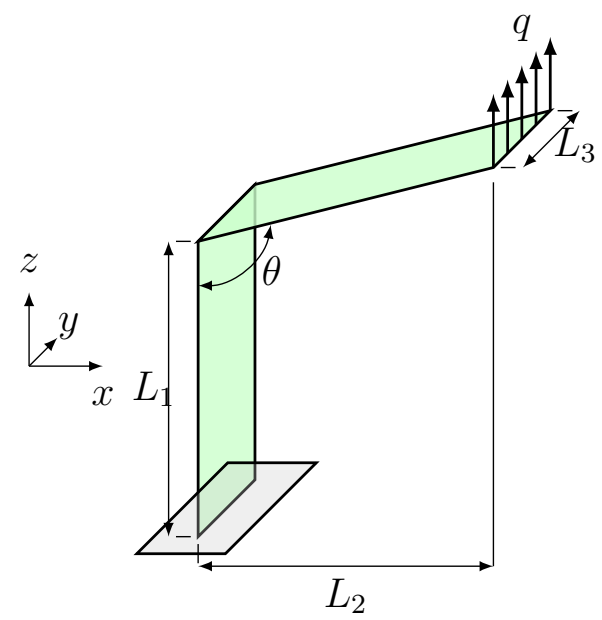

Figura 8.11: As dimensões e condições de contorno para a estrutura unida por um ângulo arbitrário $\theta$ (Dirgantara and Aliabadi, 2001).

Para este caso, os resultados segundo Dirgantara and Aliabadi (2001) para um ponto na extremidade carregada da placa superior são reunidos na Tabela 8.7.

É apresentado uma comparação com as soluções analíticas e, os erros relativos são calculados e mostrados na Tabela 8.8, 8.9 e 8.10. Todos os erros obtidos para estes casos ficaram abaixo de $5 \%$ em relação à solução analítica de Bernoulli-Euler. Além disso, com o refinando a malha, a diferença tende aos valores analíticos.

As Figuras 8.12, 8.13 e 8.14 apresentam a distribuição de deslocamento total para a estrutura com as duas placas unidas por ângulos de $91^{\circ}, 95^{\circ} \mathrm{e} 120^{\circ}$. 
Tabela 8.8: Resultados obtidos para os deslocamentos da estrutura em $L$ unida por um ângulo $\theta=91^{\circ}$.

\begin{tabular}{ccccc}
\hline $\begin{array}{c}\text { Números de } \\
\text { elementos }\end{array}$ & $\begin{array}{c}\text { Deslocamento } \\
(z)\end{array}$ & $\begin{array}{c}\text { Erro (\%) } \\
\text { Costa (2015) }\end{array}$ & $\begin{array}{c}\text { Erro (\%) } \\
\text { Euler-Bernouli }\end{array}$ & $\begin{array}{c}\text { Erro (\%) } \\
\text { Timoshenko }\end{array}$ \\
\hline 40 & 0,1533 & 4,9950 & 4,7944 & 5,0304 \\
80 & 0,1550 & 3,9415 & 3,7387 & 3,9772 \\
160 & 0,1568 & 2,8260 & 2,6208 & 2,8621 \\
400 & 0,1585 & 1,7724 & 1,5650 & 1,8089 \\
800 & 0,1593 & 1,2766 & 1,0682 & 1,3133 \\
\hline
\end{tabular}

Tabela 8.9: Resultados obtidos para os deslocamentos da estrutura em $L$ unida por um ângulo $\theta=95^{\circ}$.

\begin{tabular}{ccccc}
\hline $\begin{array}{c}\text { Números de } \\
\text { elementos }\end{array}$ & $\begin{array}{c}\text { Deslocamento } \\
(z)\end{array}$ & $\begin{array}{c}\text { Erro (\%) } \\
\text { Costa }(2015)\end{array}$ & $\begin{array}{c}\text { Erro (\%) } \\
\text { Euler-Bernouli }\end{array}$ & $\begin{array}{c}\text { Erro (\%) } \\
\text { Timoshenko }\end{array}$ \\
\hline 40 & 0,1567 & 4,9900 & 4,7995 & 5,0130 \\
80 & 0,1585 & 3,8986 & 3,7060 & 3,9219 \\
160 & 0,1628 & 2,8073 & 2,6124 & 2,8308 \\
400 & 0,1620 & 1,7765 & 1,5796 & 1,8003 \\
800 & 0,1628 & 1,2915 & 1,0936 & 1,3154 \\
\hline
\end{tabular}



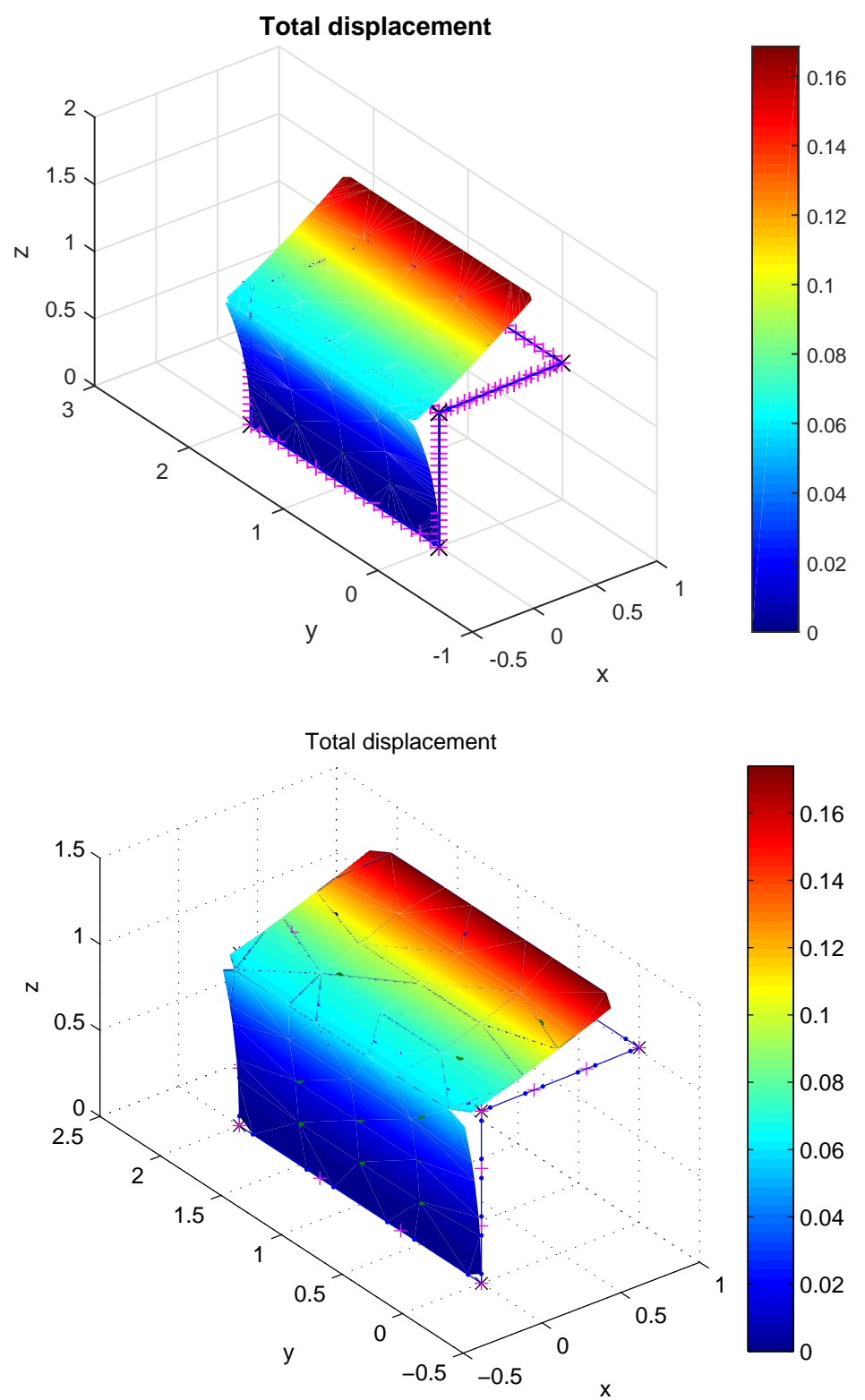

Figura 8.12: (a) Deslocamento total para a estrutura unida por um ângulo $\theta=$ $91^{\circ}$ obtido com o MEC para placa fina com 160 elementos de contorno. (b) Deslocamento total para a estrutura unida por um ângulo $\theta=91^{\circ}$ obtido com o MEC para placa espessa. 

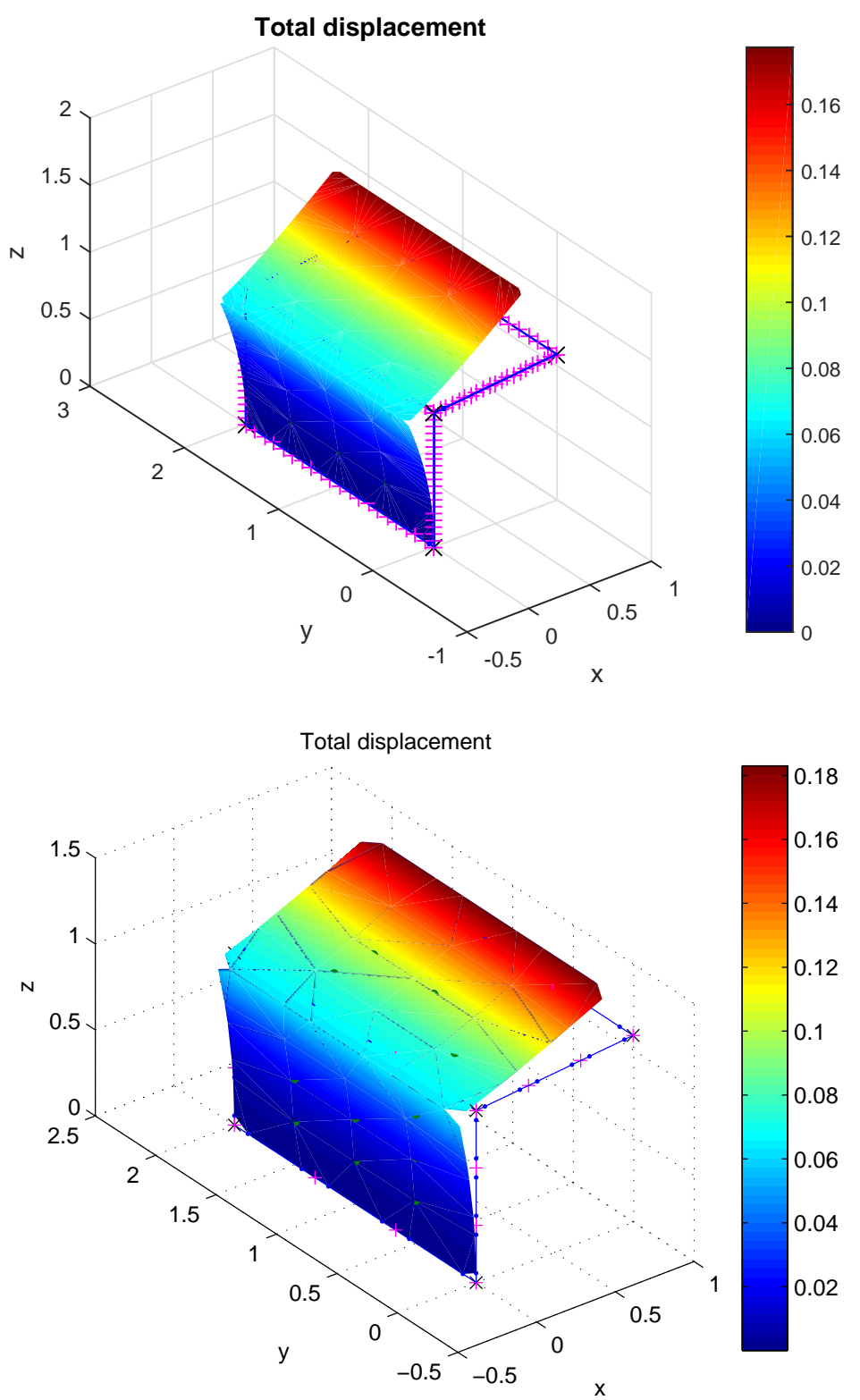

Figura 8.13: (a) Deslocamento total para a estrutura unida por um ângulo $\theta=$ $95^{\circ}$ obtido com a formulação do MEC para placa fina com 160 elementos de contorno. (b) Deslocamento total para a estrutura unida por um ângulo $\theta=95^{\circ}$ obtido com o MEC para placa espessa. 

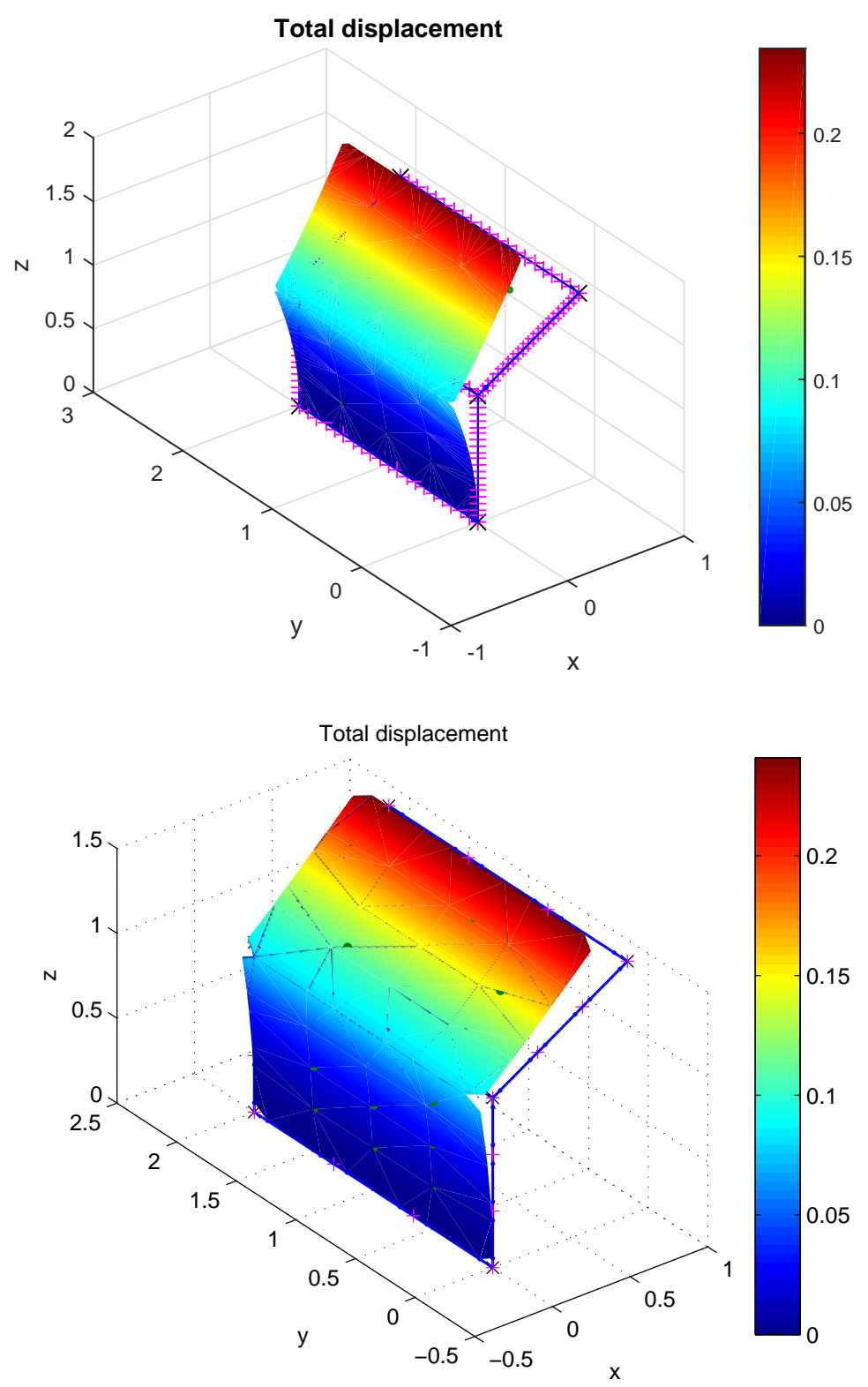

Figura 8.14: (a) Deslocamento total para a estrutura unida por um ângulo $\theta=$ $120^{\circ}$ obtido com a formulação do MEC para placa fina com 160 elementos de contorno. (b)Deslocamento total para a estrutura unida por um ângulo $\theta=120^{\circ}$ obtido com o MEC para placa espessa. 
Tabela 8.10: Resultados obtidos para os deslocamentos da estrutura em $L$ unida por um ângulo $\theta=120^{\circ}$.

\begin{tabular}{ccccc}
\hline $\begin{array}{c}\text { Números de } \\
\text { elementos }\end{array}$ & $\begin{array}{c}\text { Deslocamento } \\
(z)\end{array}$ & $\begin{array}{c}\text { Erro (\%) } \\
\text { Costa }(2015)\end{array}$ & $\begin{array}{c}\text { Erro (\%) } \\
\text { Euler-Bernouli }\end{array}$ & $\begin{array}{c}\text { Erro (\%) } \\
\text { Timoshenko }\end{array}$ \\
\hline 40 & 0,1567 & 4,9324 & 4,7706 & 4,8920 \\
80 & 0,1586 & 3,7797 & 3,6159 & 3,7388 \\
160 & 0,1604 & 2,6876 & 2,5220 & 2,6463 \\
400 & 0,1622 & 1,5956 & 1,4281 & 1,5538 \\
800 & 0,1630 & 1,1102 & 0,9420 & 1,0682 \\
\hline
\end{tabular}

\subsubsection{Viga em formato de caixa}

Este modelo é formado por quatro placas dispostas de modo a se posicionarem como a parte lateral de uma caixa. As arestas localizadas no plano $(x-z)$ em $y=0$ possuem deslocamentos restritos em todas as direções. São impostos dois tipos de carregamentos como mostrado na Figura 8.15 (o primeiro fletindo a caixa e o segundo torcendo). A indicação das dimensões geométricas são dadas por: $L_{1}=0,8 \mathrm{~m}, L_{2}=2 \mathrm{~m}, L_{3}=0,2 \mathrm{~m}$, $0,1 \mathrm{~m}$ e $0,05 \mathrm{~m}$. A espessura das placas é $t_{1}=2,0 \mathrm{~mm}$. A carga distribuída ao longo das arestas indicadas na Figura 8.15 tem valor resultante $F=5000 \mathrm{~N}$. As propriedades mecânicas são $E=70 \mathrm{GPa}$ e $\nu=0,3$.

O modelo foi discretizado usando quatro sub regiões, cada uma contendo no máximo 3200 elementos de contorno constantes. A Figura 8.16 apresenta a distribuição de deslocamento total na estrutura sujeita aos carregamentos indicados na Figura 8.15(a) para um valor de $L_{3}=0,05 \mathrm{~m}$.

Os resultados para o primeiro tipo de carregamento (carregamento de flexão) são apresentados nas Figuras 8.17, 8.18 e 8.19. Estes resultados são comparados com o MEF (Ansys), a formulação de placas espessas e a solução analítica de Bernoulli-Euler.

O acordo dos resultados obtidos pela formulação proposta com a solução analítica parece estar relacionado à razão de aspecto da caixa $\left(L_{2} / L_{3}\right)$. Com seu aumento, a solução deste trabalho desvia dos resultados obtidos com o MEF (Ansys). Mesmo assim, quando comparados, os resultados apresentam uma boa concordância.

Os resultados para o segundo tipo de carregamento (carregamento de torção) são apresentados nas Figuras 8.20, 8.21 e 8.22. Estes resultados são comparados àqueles obtidos com o MEF (Ansys). A boa concordância entre os resultados só foi alcançada com o aumento da espessura $L_{3}$, conforme visto na Figura 8.21. Considerando o número elevado de elementos, uma hipótese para a não convergência nos casos de pequenos 


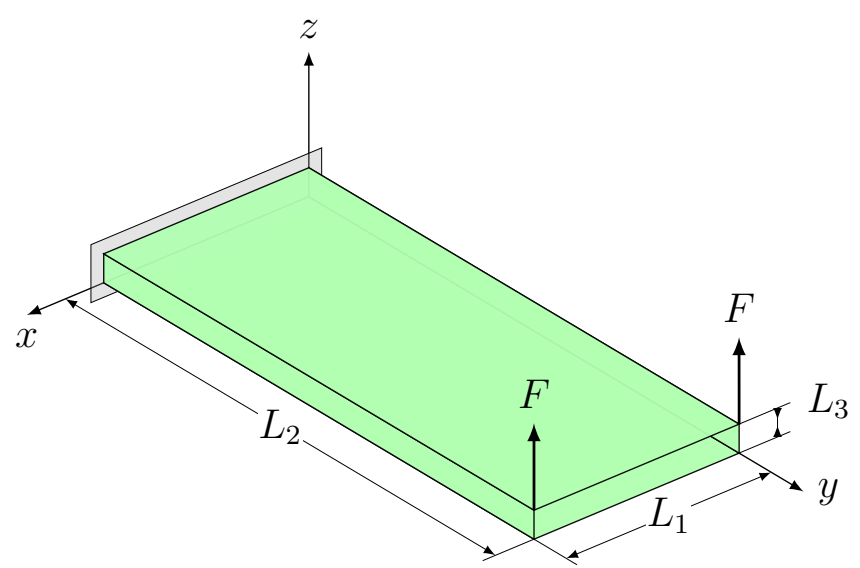

(a)

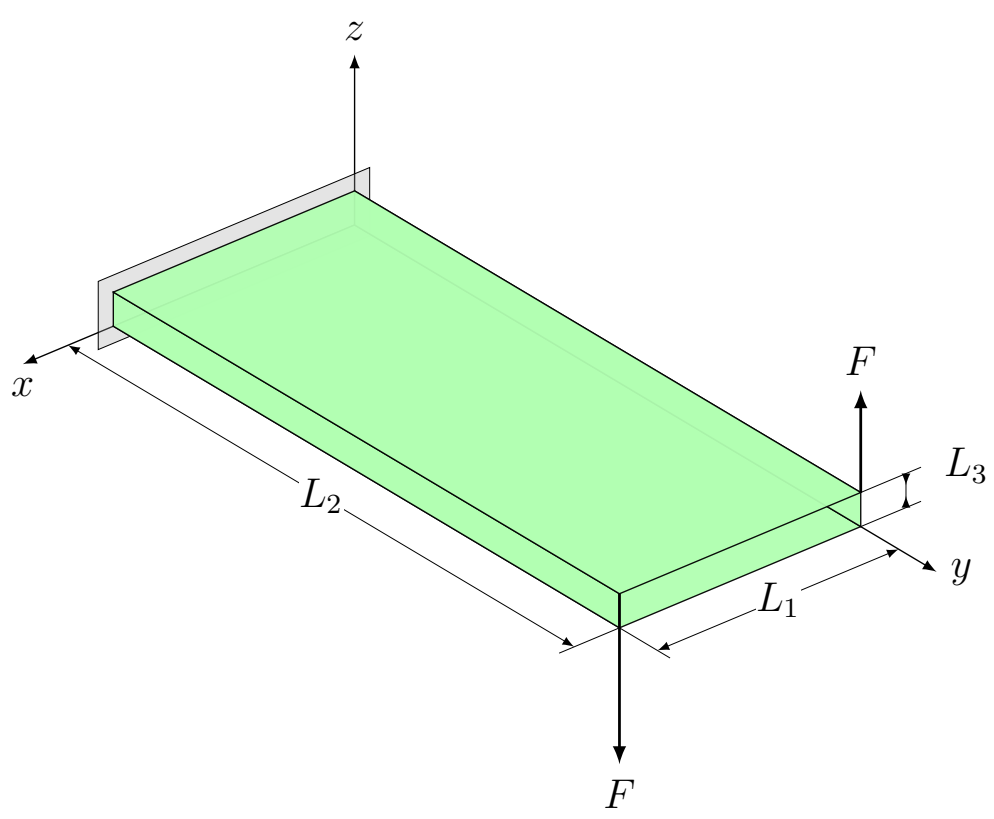

(b)

Figura 8.15: Dimensões e condições de contorno para a viga em forma de caixa em balanço.

valores de $L_{3}$ é o tipo de elemento adotado, constante. Uma investigação com elementos de maior ordem (quadrático por exemplo), torna-se necessário. Entretanto, esta análise ficou fora do escopo deste trabalho. 


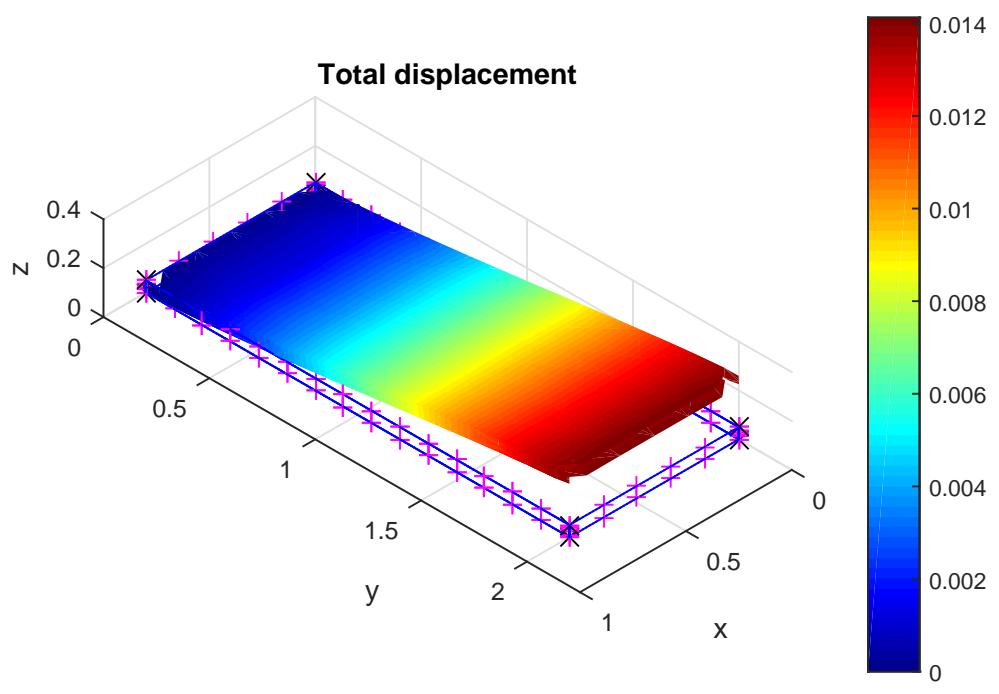

Figura 8.16: Deslocamento total para a viga em formato de caixa em balanço para $L_{3}=0,05 \mathrm{~m}$ obtido com MEC para placas finas.

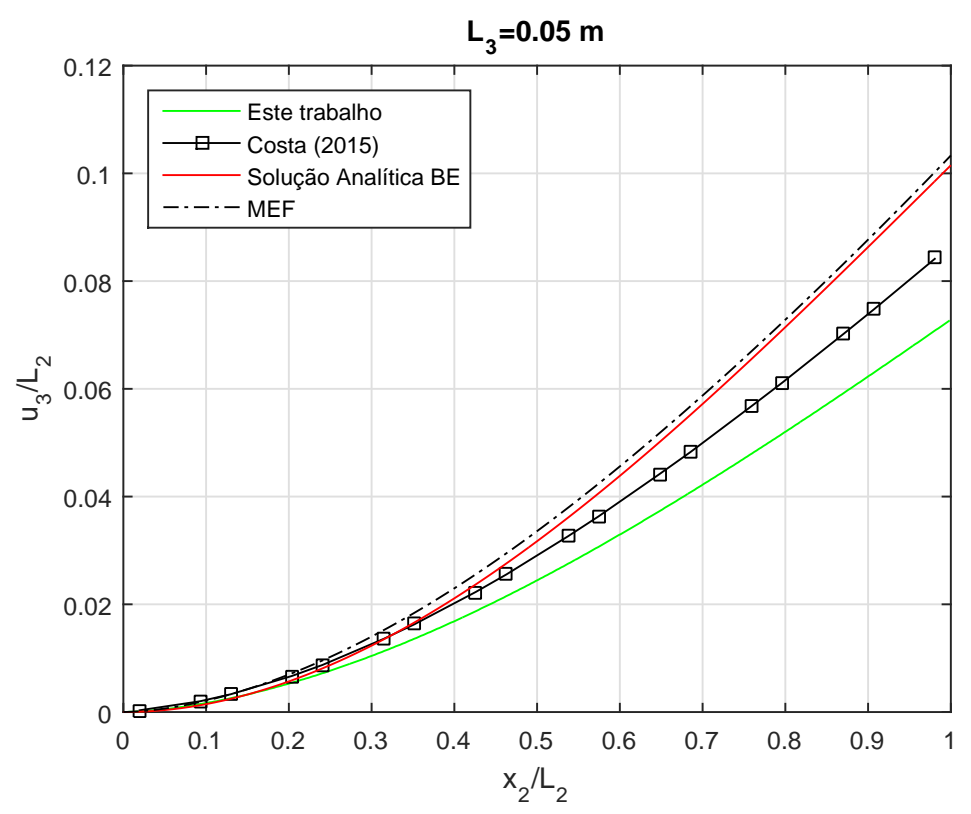

Figura 8.17: Deslocamento na direção $z$ para a viga em formato de caixa em balanço para $L_{3}=0,05 \mathrm{~m}$ com 96 elementos. 


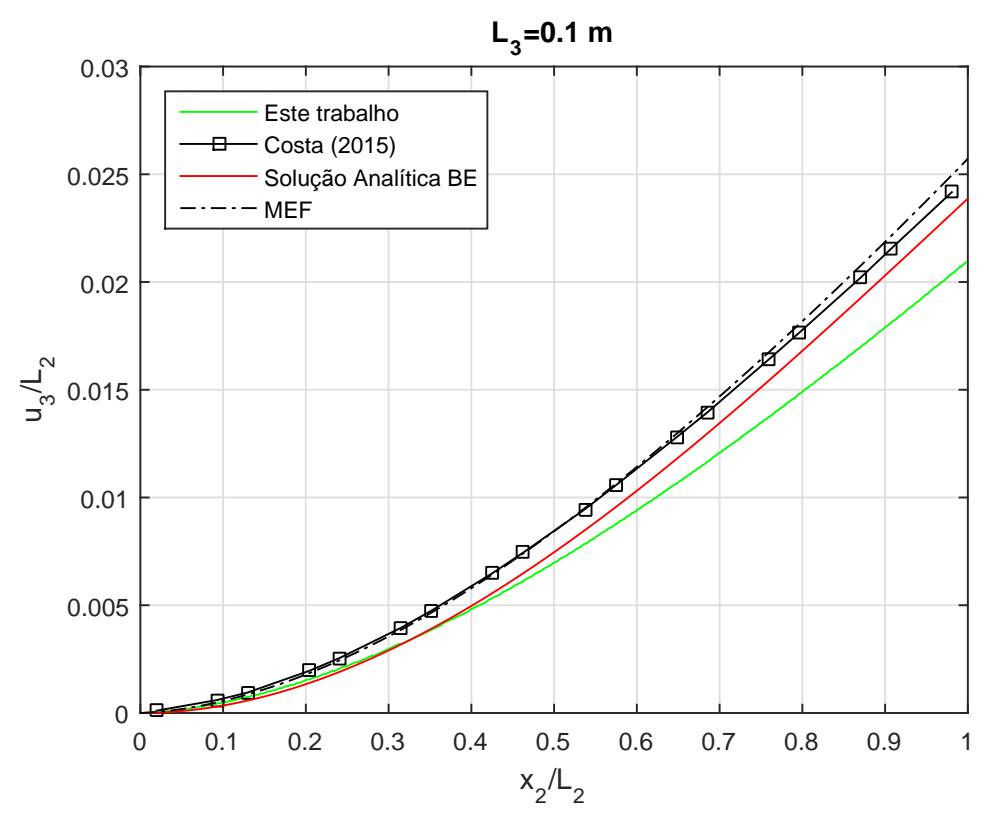

Figura 8.18: Deslocamento na direção $z$ para a viga em formato de caixa em balanço para $L_{3}=0,1 \mathrm{~m}$ com 192 elementos.

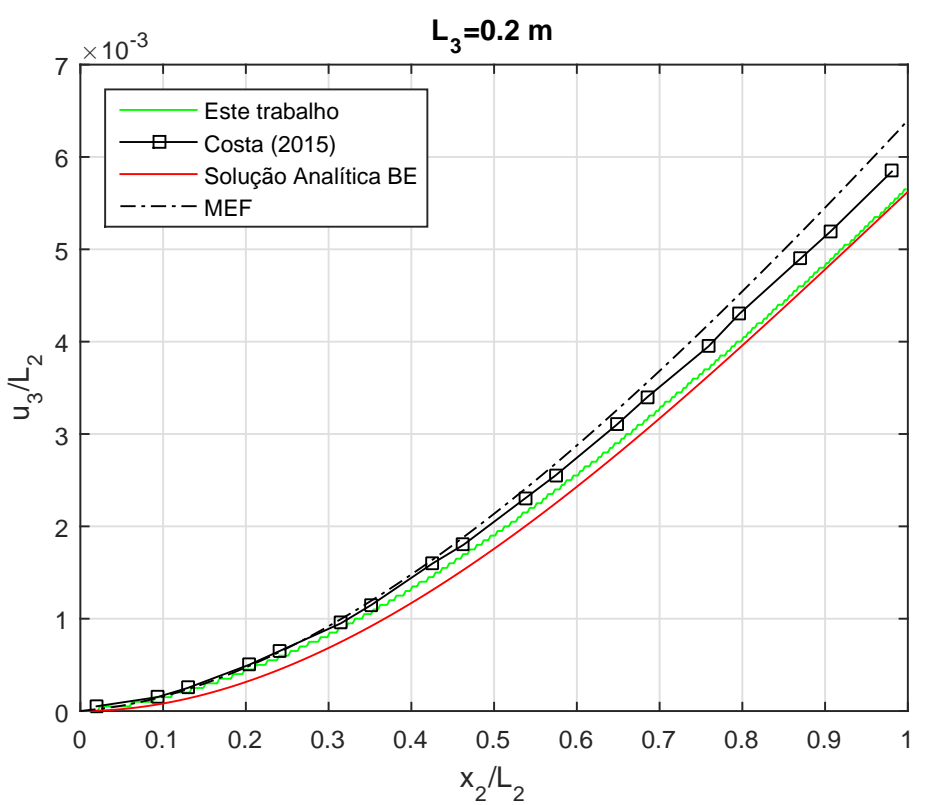

Figura 8.19: Deslocamento na direção $z$ para a viga em formato de caixa em balanço para $L_{3}=0,2 \mathrm{~m}$ com 96 elementos. 


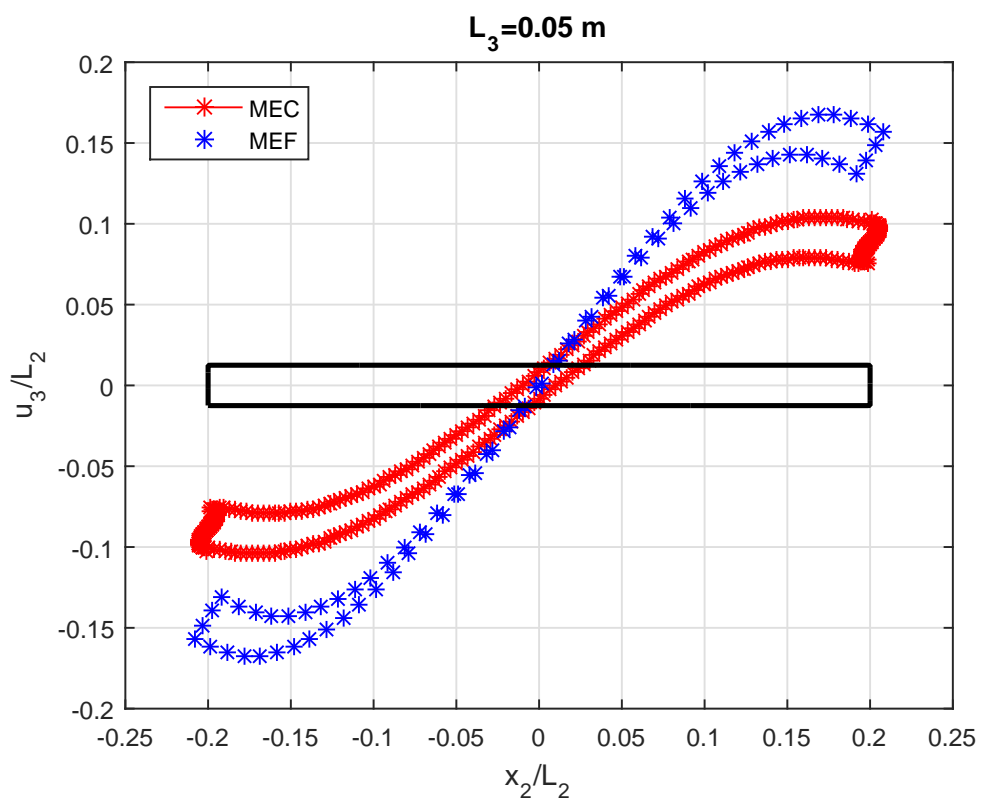

Figura 8.20: Deslocamentos $u_{2}$ para o lado alinhado com a direção $x_{2}$ localizados nas coordenadas $x_{1}=x_{3}=0 \mathrm{~m}$ para $L_{3}=0,05 \mathrm{~m}$.

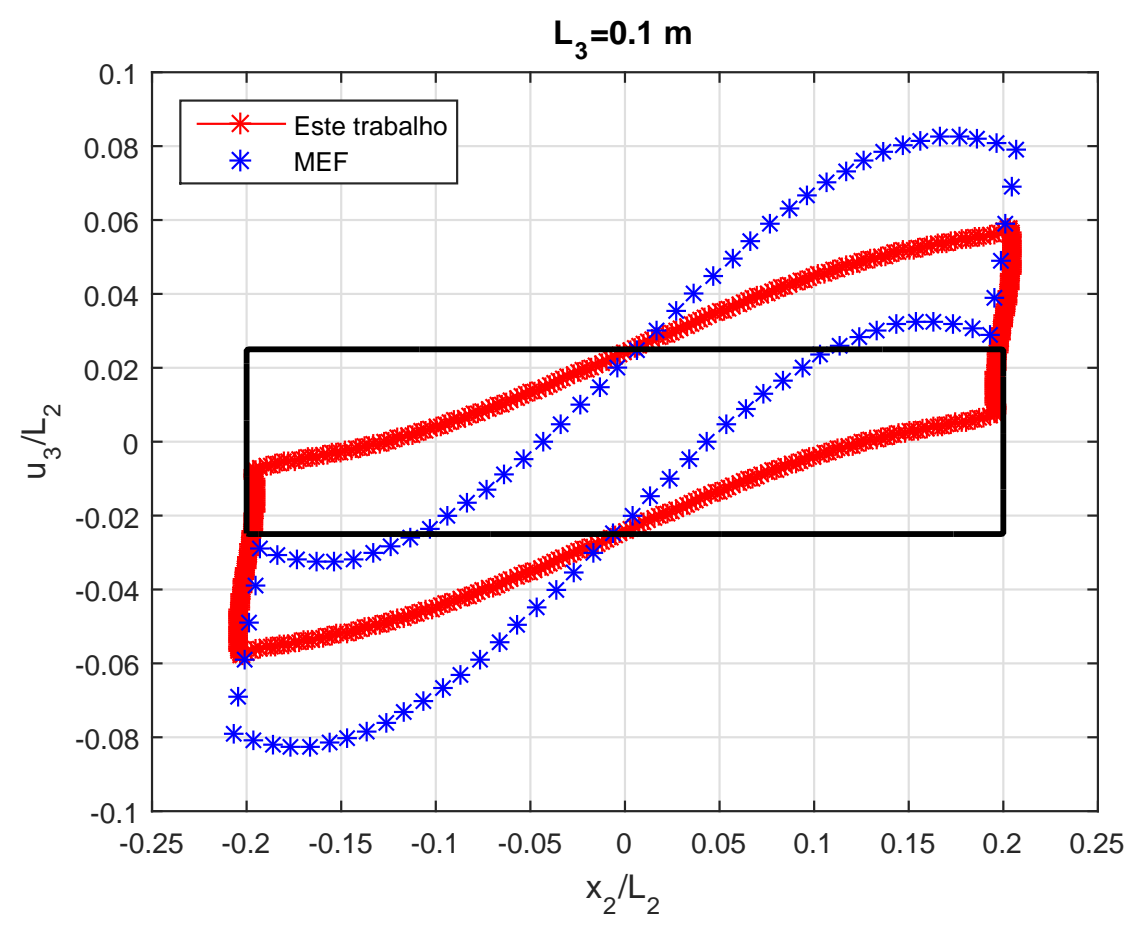

Figura 8.21: Deslocamentos em $x_{2}=2 \mathrm{~m}$ para a viga em formato de caixa em balanço sujeita a um momento para $L_{3}=0,1 \mathrm{~m}$. 


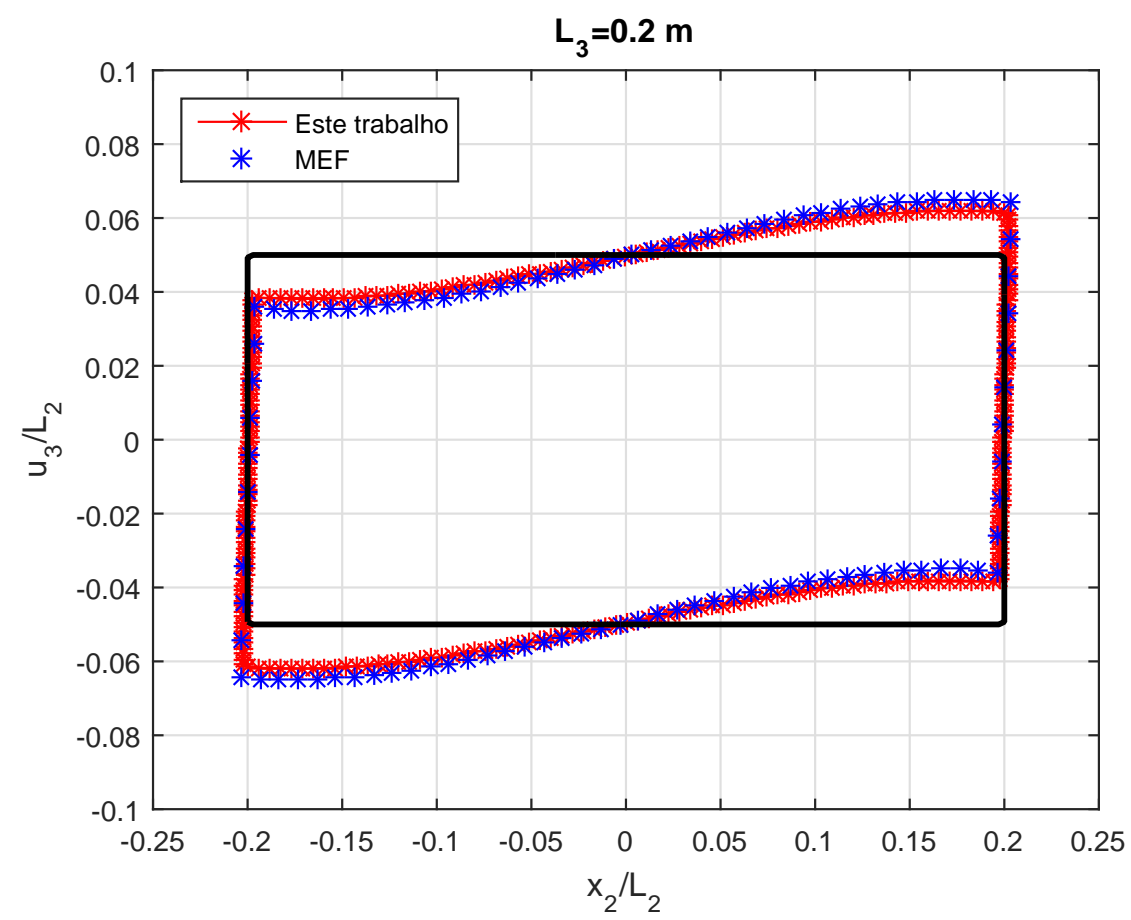

Figura 8.22: Deslocamentos em $x_{2}=2 \mathrm{~m}$ para a viga em formato de caixa em balanço sujeita a um momento para $L_{3}=0,2 \mathrm{~m}$. 


\subsection{Associação em $L$ com carregamento lateral}

Este problema foi simulado com o intuito de avaliar o caso em que a deslocamento na direção y é relevante no que diz respeito à imposição das condições de compatibilidade. Este modelo foi discretizado usando duas sub-regiões, cada uma contendo 360 elementos de contorno constantes. As propriedade geométricas são: $L_{1}=1,0 \mathrm{~m}, L_{2}=2,0 \mathrm{~m}$, $L_{3}=1,0 \mathrm{~m}$ e espessura $t=0,1 \mathrm{~m}$. As propriedades mecânicas são: $E=100 \mathrm{kPa}$, $\nu=0,0$ e $q=10 \mathrm{~N}$. Como é possível observar, o carregamento colocado na lateral impõe uma rotação ao redor do eixo $z$ da placa superior. Os resultados são apresentados na Figura 8.24.

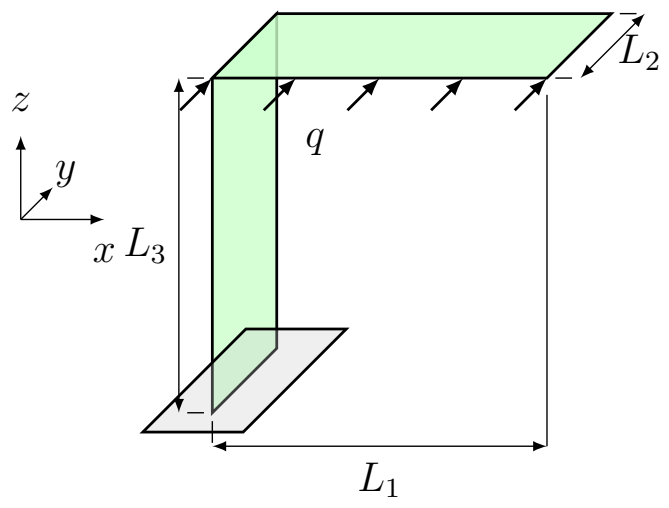

Figura 8.23: Estrutura L-shaped.

Para que seja possível comparar os resultados obtidos com a formulação do MEC deste trabalho, MEC para placa espessa e MEF (Ansys), a Figura 8.25 é apresentada. Neste caso, foi usado uma malha com 2400 elementos de contorno constantes. Este gráfico mostra os deslocamentos nas direções $x_{1}$ e $x_{2}$ para a placa superior, projetados no plano $x_{1}-x_{2}$. Indica claramente que os resultados obtidos com a formulação apresentada neste trabalho tem um bom acordo com a solução obtida com MEF (Ansys). A formulação produziu valores que são apresentados na (Figura 8.24). 


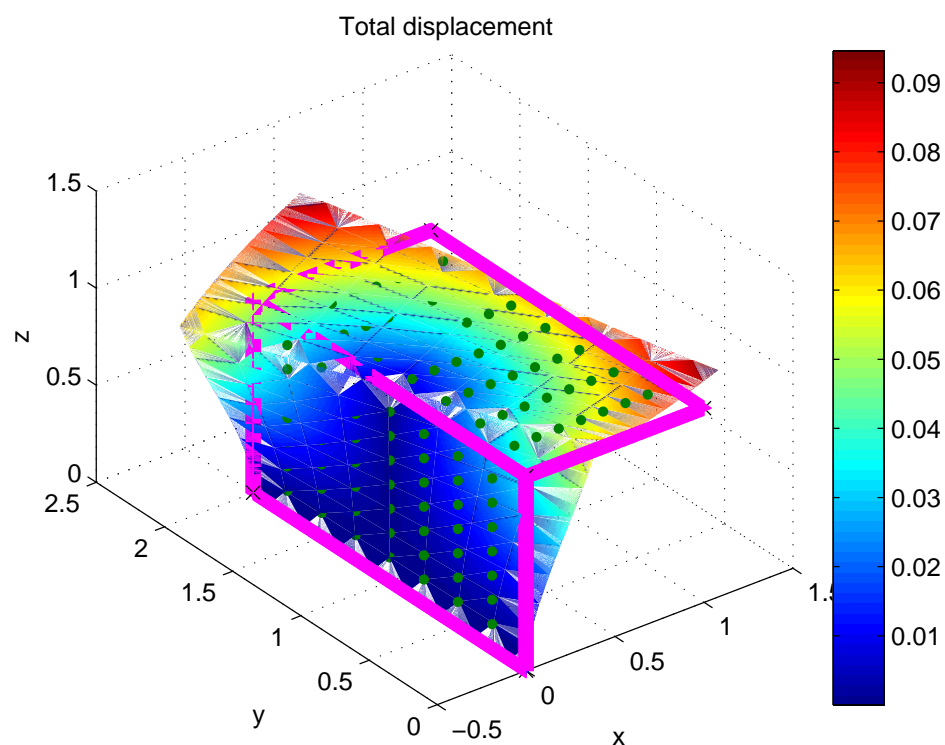

(a)

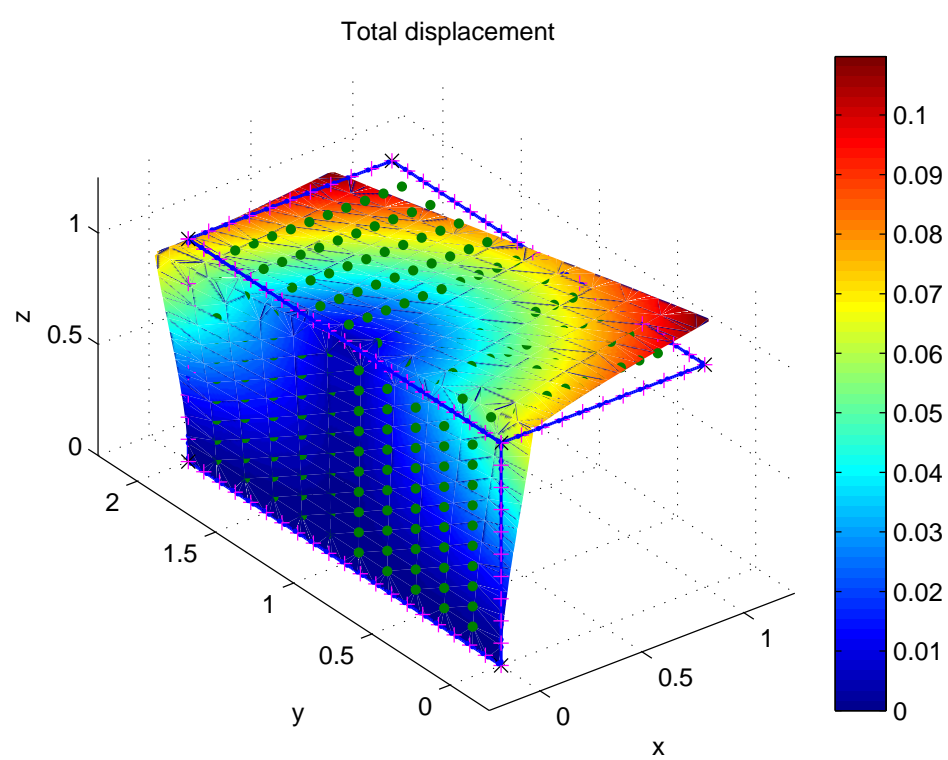

(b)

Figura 8.24: Resultados obtidos com MEC: a) placa fina e b) placa espessa. 


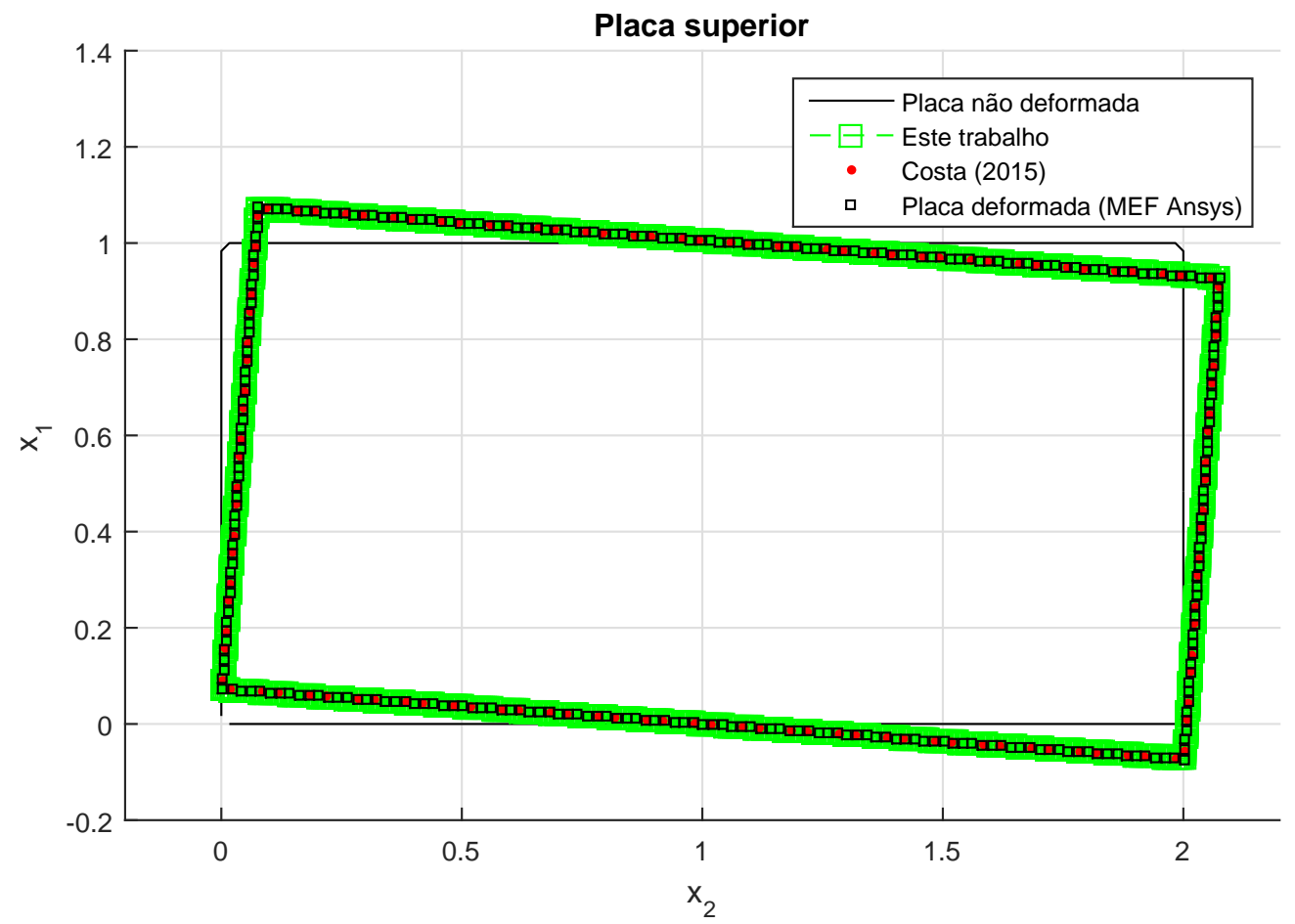

Figura 8.25: Resultados para a placa superior projetados no plano $x_{1}-x_{2}$. 


\section{Capítulo 9}

\section{Resultados Numéricos de Problemas Transientes}

\subsection{Introdução}

Neste Capítulo, o método dos elementos de contorno será aplicado ao cálculo dos campos de deslocamentos para problemas transientes no domínio do tempo. Estes resultados são comparados com soluções obtidas pelo método sem malhas PetrovGalerkin (Meshless Petrov Galerkin Method, MLPG) proposto por Sladek et al..

\subsection{Resultados numéricos}

\subsubsection{Placa apoiada}

A Figura 9.1 mostra o problema físico considerado: uma placa simplesmente apoiada nos quatro lados, submetida a carregamento constante distribuído uniformemente por toda a superfície. As propriedades geométricas são: $L=1 \mathrm{~m}$ e espessura $t=$ $0,0127 \mathrm{~m}$. As propriedades do material são: módulo de elasticidade $E=6,895$ GPa, razão de Poisson $\nu=0,3$, densidade $\rho=7,16610^{4} \mathrm{~N} / \mathrm{m}^{3}$ e carregamento $q=$ $2,0710^{6} \mathrm{~N} / \mathrm{m}^{2}$.

Neste caso, foram usados uma malha com 20 e 24 elementos de contorno constantes para 1 e 2 sub-regiões, respectivamente. A deflexão dinâmica é normalizada pelo deslocamento do problema estático $w_{3}^{\text {sta }}=0,0286$. O tempo inicial é normalizado por $t_{0}=a^{2} / 4 \sqrt{\rho h / D}=0,0135 \mathrm{~s}$ e passo de tempo $\Delta \tau=4,509910^{-4} \mathrm{~s}$. A variação no tempo do deslocamento no ponto central é observado na Figura 9.2. Nota-se uma boa concordância com os resultados do trabalho de Sladek et al.. 


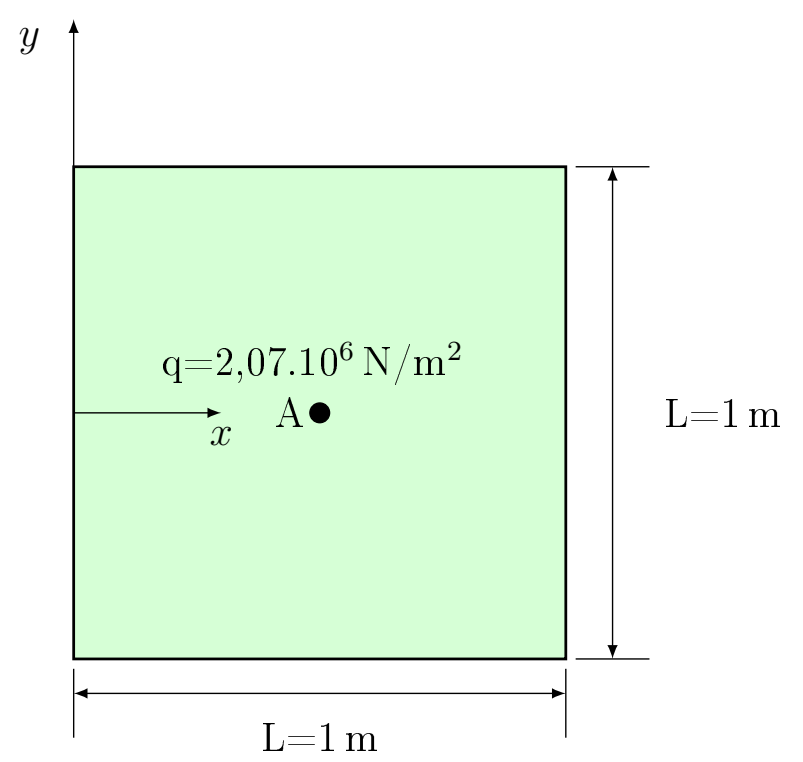

Figura 9.1: Ilustração da placa apoiada.

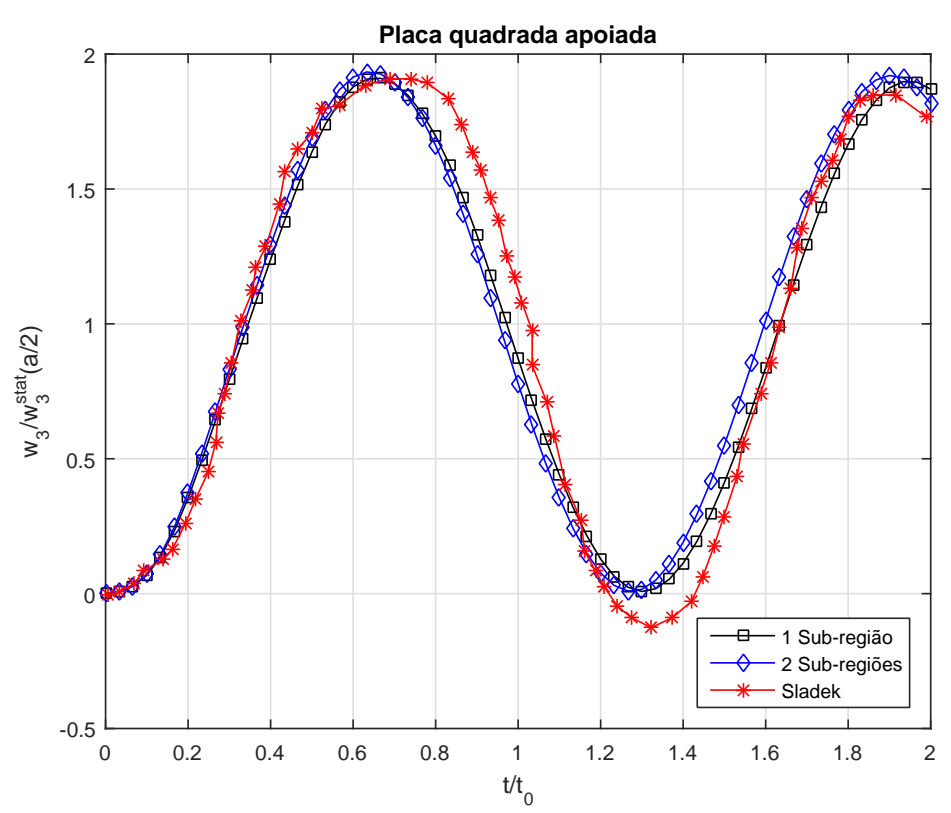

Figura 9.2: Variação no tempo do deslocamento no ponto central da placa quadrada simplesmente apoiada submetido a uma carga repentinamente aplicada. 


\subsubsection{Placa engastada}

A Figura 9.3 mostra o problema físico considerado: uma placa engatada nos quatro lados, submetida a carregamento constante distribuído uniformemente por toda a superfície. As propriedades geométricas são: $L=1 \mathrm{~m}$ e espessura $t=0,0127 \mathrm{~m}$. As propriedades do material são: módulo de elasticidade $E=6,895 \mathrm{GPa}$, razão de Poisson $\nu=0,3$, densidade $\rho=7,16610^{4} \mathrm{~N} / \mathrm{m}^{3}$ e carregamento $q=2,0710^{6} \mathrm{~N} / \mathrm{m}^{2}$.

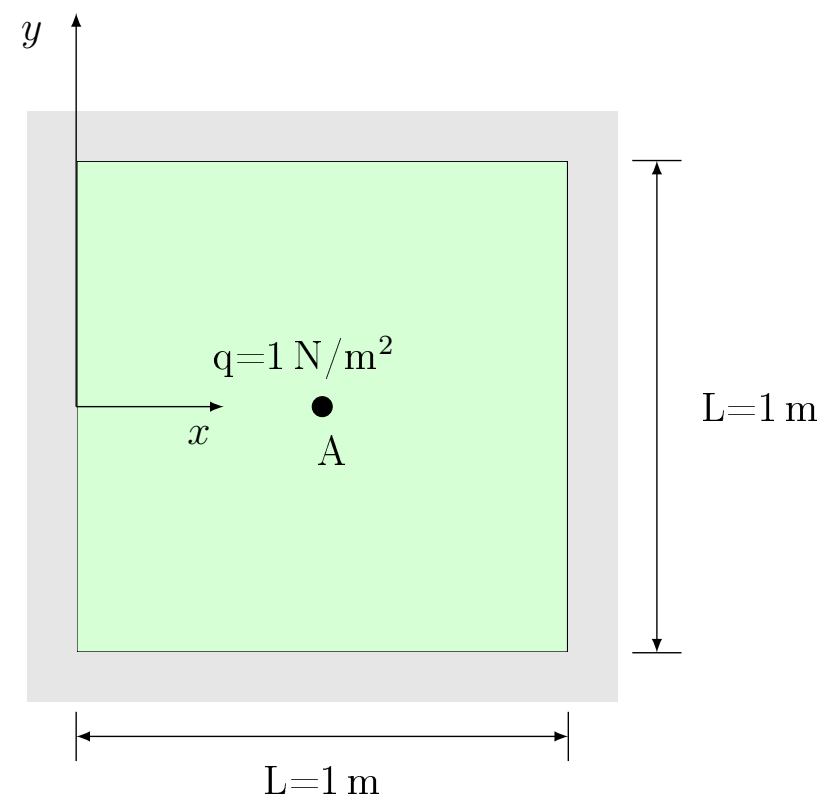

Figura 9.3: Ilustração da placa engastada.

Foram usados uma malha com 12 e 8 elementos de contorno constantes para 1 e 2 sub-regiões, respectivamente. A deflexão dinâmica é normalizada por um coeficiente estático $w_{3}^{\text {sta }}=0,00126 C a^{4} / D=0,0084$. O tempo inicial é normalizado por $t_{0}=$ $a^{2} / 4 \sqrt{\rho h / D}=0,0135 \mathrm{~s}$ e passo de tempo $\Delta \tau=2,319410^{-4} \mathrm{~s}$. A variação no tempo do deslocamento no ponto central é observado na Figura 9.4, onde também se observa uma boa concordância com os resultados de Sladek et al.. 


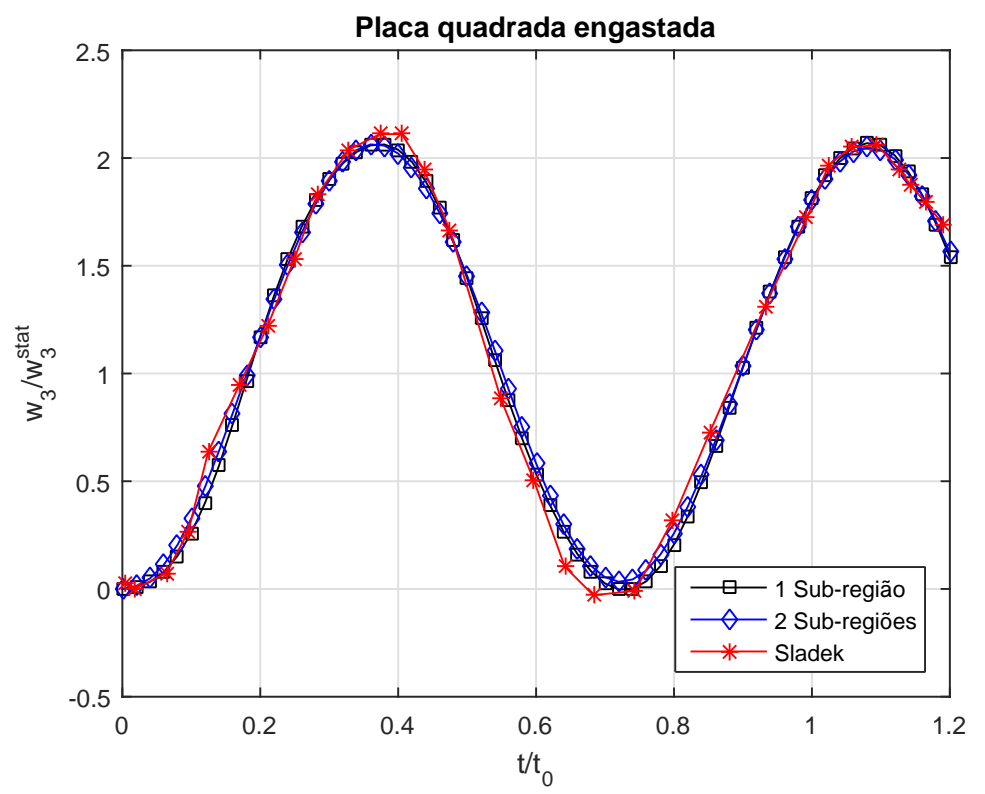

Figura 9.4: Variação no tempo do deslocamento no ponto central da placa quadrada engastada submetido a uma carga repentinamente aplicada. 


\subsubsection{Placa em balanço}

A Figura 9.5 mostra o problema físico considerado: uma placa engatada em um dos lados e livre nos demais lados, submetida a carregamento constante distribuído uniformemente na aresta oposta ao engaste. As propriedades geométricas são: $L=1 \mathrm{~m}$ e espessura $t=0,0127 \mathrm{~m}$. As propriedades do material são: módulo de elasticidade $E=6,895 \mathrm{GPa}$, razão de Poisson $\nu=0,3$, densidade $\rho=7,16610^{4} \mathrm{~N} / \mathrm{m}^{3}$. A carga é distribuída ao longo da aresta, orientada na direção $y$ com valor $q=2,0710^{6} \mathrm{~N} / \mathrm{m}^{2}$.
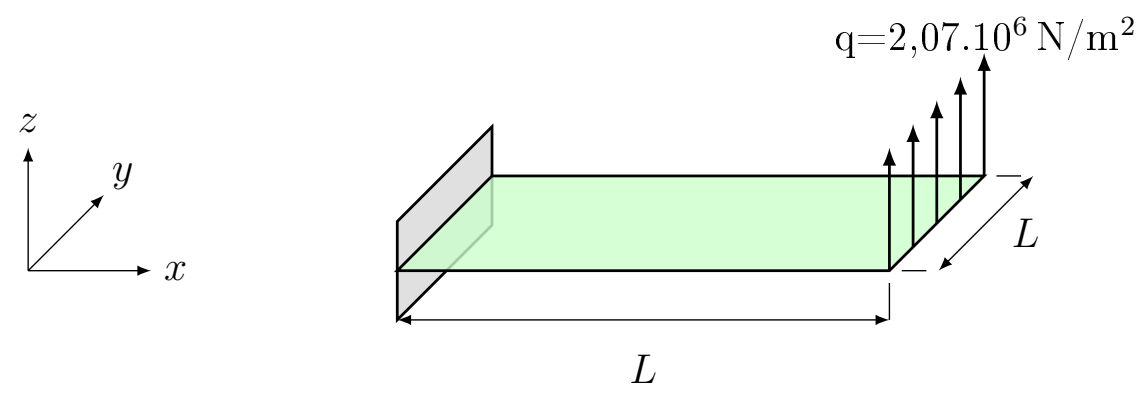

Figura 9.5: Ilustração da placa em balanço.

Simulou-se uma malha com 12 e 8 elementos de contorno constantes para 1 e 2 sub-regiões, respectivamente. A deflexão dinâmica é normalizada por um coeficiente estático $w_{3}^{\text {sta }}=0,00126 C a^{4} / D=1,08.10-9$. O tempo inicial é normalizado por $t_{0}=L / c_{2}=6,6890.10^{-5} \mathrm{~s}$, velocidade de onda $c_{2}=2990 \mathrm{~m} / \mathrm{s}$ e passo de tempo $\Delta \tau=$ $5,5741 \cdot 10^{-5} \mathrm{~s}$. A variação no tempo do deslocamento no ponto central é observado na Figura 9.6. Mais uma vez, há uma boa concordância com o resultado apresentado por Sladek et al.. 


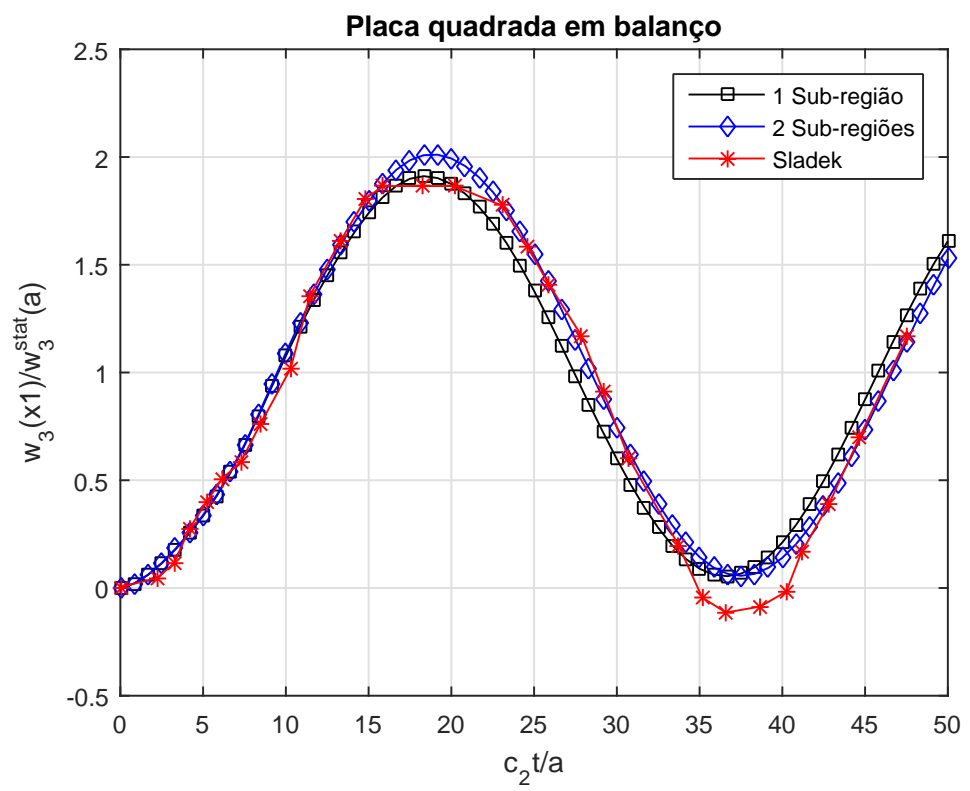

Figura 9.6: Variação no tempo do deslocamento transversal no ponto $A$ da placa quadrada em balanço submetido a uma carga repentinamente aplicada. 


\subsubsection{Tira infinita sob tração uniforme}

Considere uma tira de comprimento infinito (Figura 9.7) carregada num instante $\tau_{o}=0 \mathrm{~s}$ por uma tensão de tração $\sigma_{o}=1 \mathrm{~Pa}$ tipo degrau, conforme a Figura 9.9. Devido a simetria, apenas uma parte da tira precisa ser modelada, conforme mostrado na Figura 9.8. Um estado plano de deformação é assumido. O material é considerado isotrópico com as seguintes propriedades de material: módulo de elasticidade $E=10,6810^{4} \mathrm{~Pa}$, coeficiente de Poisson $\nu=0,3333$ e densidade $\rho=1 \mathrm{Kg} / \mathrm{m}^{3}$. Este problema é equivalente ao problema isotrópico em estado plano de deformação analisado por Dominguez (1993) usando diferentes formulações de elementos de contorno para elasto-dinâmica.

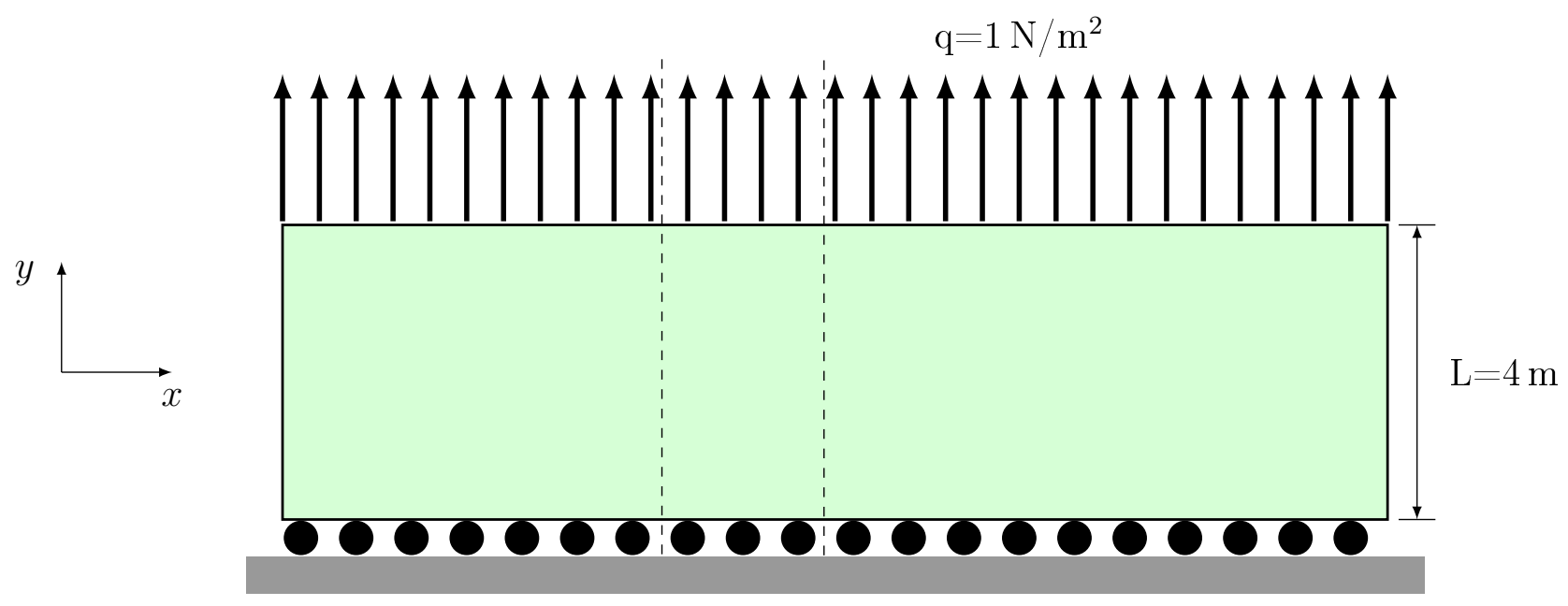

Figura 9.7: Ilustração da tira infinita sob tração uniforme.

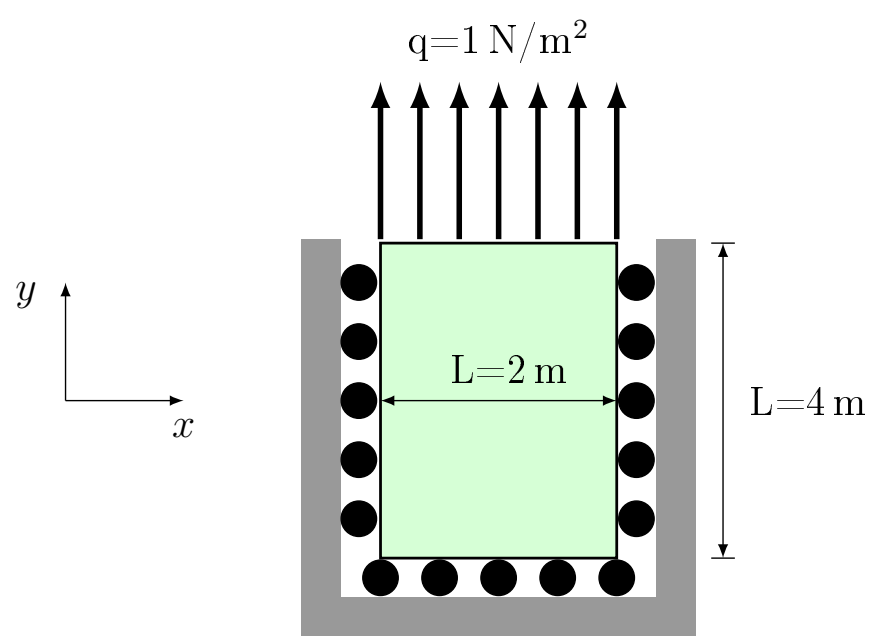

Figura 9.8: Ilustração da seção da tira infinita sob tração uniforme.

A tira foi discretizada usando-se 1 e 2 sub-regiões. O problema foi analisado com 3 nós interno, 6 e 12 elementos de contorno constante para 1 e 2 sub-regiões, respecti- 


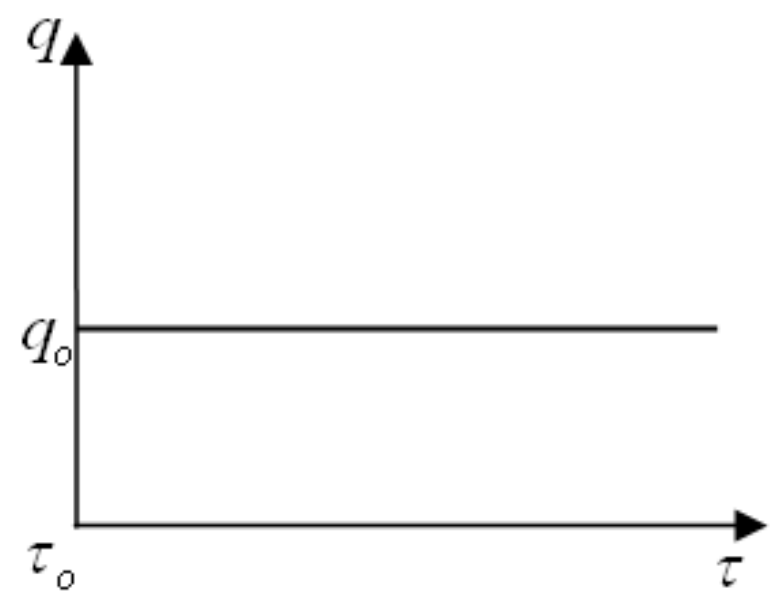

Figura 9.9: Carregamento tipo função degrau.

vamente, e passo de tempo $\Delta \tau=7,2210^{-4} \mathrm{~s}$. A Figura 9.10 mostra o deslocamento vertical do nó central da borda livre da tira comparada com a solução analítica. Pode-se observar uma boa concordância com os resultados obtidos por Dominguez (1993).

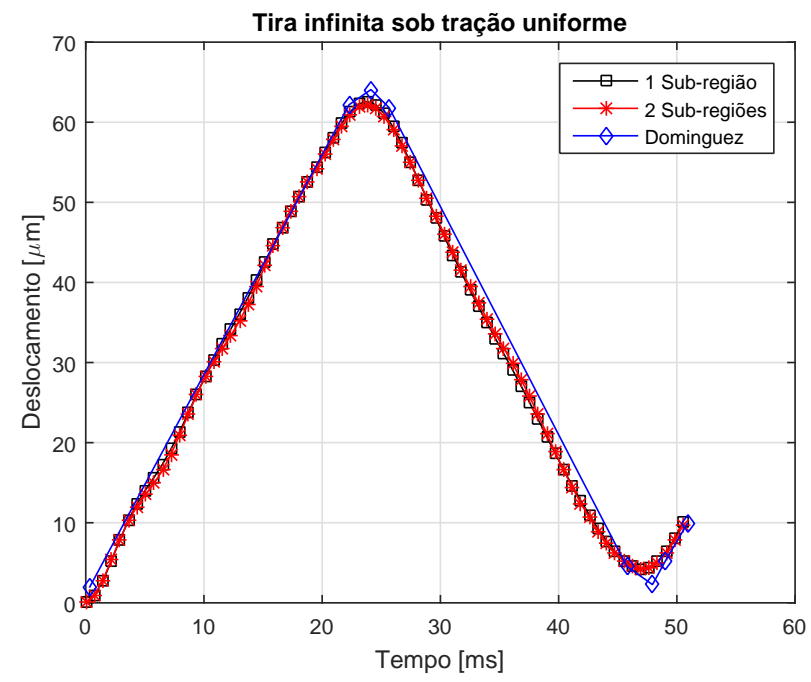

Figura 9.10: Variação do deslocamento no tempo da tira infinita sob carga com passo de tempo uniforme. 


\subsubsection{Associação em $L$}

Este modelo é composto por duas placas associadas de maneira a formar uma estrutura em $L$. As propriedades e dimensões são dadas na Figura 9.11, onde $L_{1}=1 \mathrm{~m}$, $L_{2}=1 \mathrm{~m}, L_{3}=2 \mathrm{~m}, t_{1}=0,008 \mathrm{~m}$ e $t_{2}=0,008 \mathrm{~m}$. A carga é distribuída ao longo da aresta superior, orientada na direção $z$ com valor $q=100 \mathrm{~N} / \mathrm{m}^{2}$. As propriedades mecânicas são: $E=210 \mathrm{GPa}, \rho=7800 \mathrm{~N} / \mathrm{m}^{3}$ e $\nu=0,3$.

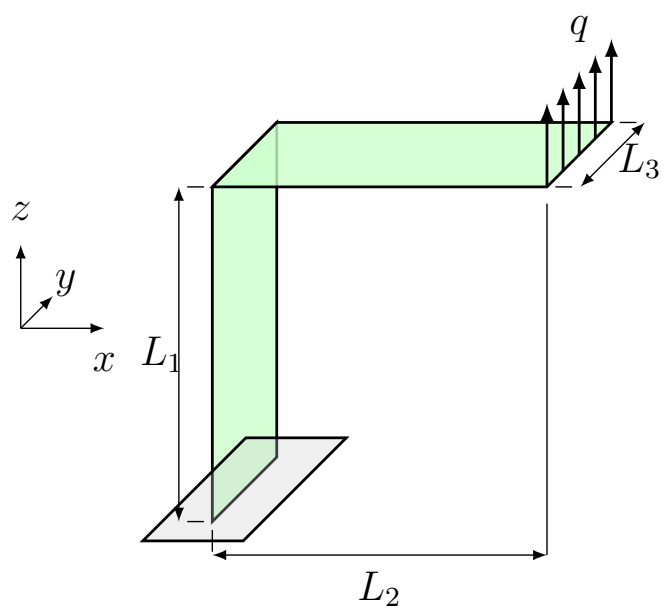

Figura 9.11: Dimensões e condições de contorno para a estrutura em $L$ Dirgantara and Aliabadi (2001). 
A estrutura foi discretizada usando-se 300 elementos de contorno constantes e passo de tempo $\Delta \tau=0,0100 \mathrm{~s}$. A Figura 9.12 mostra o deslocamento vertical da borda livre da placa associada em $L$.

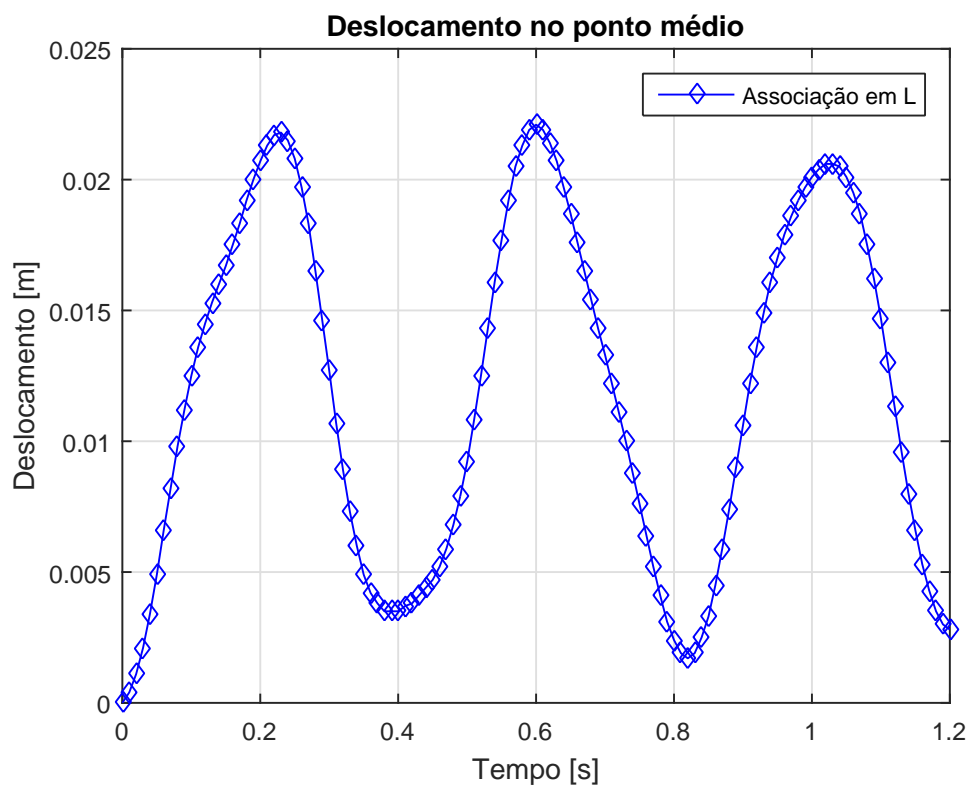

Figura 9.12: Variação do deslocamento no tempo da tira infinita sob carga com passo de tempo uniforme.

Este é um problema original, que não possui similar na literatura para se comparar com o resultado obtido. Entretanto, o valor máximo do deslocamento é o esperado para este tipo de problema. É bem conhecido da literatura que para problemas transiente sem amortecimento sob carga tipo Heaviside, o valor máximo de deslocamentos, tensões e deformações é duas vezes os respectivos valores de problemas estáticos Campos (2012). Neste caso, o resultado estático para o deslocamento é igual a $w_{e}=10,2 \mathrm{~mm}$. Por sua vez, o valor máximo para o problema dinâmico foi de $w_{d}=21,5 \mathrm{~mm}$. Desta forma, o erro relativo foi de:

$$
\operatorname{Err} .(\%)=\frac{\left|w_{e}-w_{d}\right|}{\left|w_{e}\right|} \times 100 \%=5,74 \%
$$




\section{Capítulo 10}

\section{Considerações finais}

\subsection{Conclusões}

Uma formulação do método dos elementos de contorno para o cálculo de deslocamentos em problemas isotrópicos foi desenvolvida para análise de estruturas constituídas pela associação de placas planas no espaço sob carregamentos estáticos e dinâmicos. As formulações de elasticidade plana e de flexão de placas finas (placas de Kirchhoff) foram usadas simultaneamente. Com isso, obteve-se uma formulação com quatro graus de liberdade por nó, sendo os dois deslocamentos provenientes da formulação de elasticidade plana e, o deslocamento transversal e a rotação normal da formulação de placas finas. As variáveis desconhecidas foram assumidas constante ao longo de cada elemento (elemento de contorno constante). Integrais de domínio que surgem devido a cargas distribuídas foram transformadas de forma exata para integrais de contorno. $\mathrm{Na}$ formulação dinâmica, os termos de inércia foram considerados como forças de corpo, gerando assim, integrais de domínio na formulação. Estas integrais de domínio foram transformadas em integrais de contorno usando o método da integração radial. Para problemas dinâmicos, graus de liberdade no interior do domínio foram inseridos de modo a melhorar a aproximação dos termos de inércia. Entretanto, os pontos internos podem ser inseridos de maneira aleatória e nenhuma discretização do domínio é necessária. Obteve-se, assim, uma formulação para problemas estáticos e dinâmicos onde somente os contornos das placas são discretizados. Não foi encontrada na literatura, formulações do método dos elementos de contorno onde somente o contorno é discretizado para problemas dinâmicos de associação de placas planas no espaço. Por esta razão, está foi a principal contribuição deste trabalho.

No arranjo tridimensional, cada placa foi definida como uma sub-região e os termos locais foram calculados de maneira independente em coordenadas locais. As condições de compatibilidade de equilíbrio e deslocamentos foram impostas, acoplando assim 
todas as sub-regiões. Finalmente, o sistema global foi montado e as condições de contorno impostas. No caso de problemas dinâmicos (transientes), o método de Houbolt foi usado para aproximar as acelerações nodais como uma função dos deslocamentos nodais.

No método da integração radial, foi utilizada como função de aproximação a função de base radial spline de placas finas que, conforme relatado na literatura, apresenta um bom desempenho em várias outras formulações.

Vários testes numéricos foram utilizados para validação. Inicialmente para problemas planos, depois problemas com associação de placas no espaço. Em ambos os casos, foram analisadas a sensibilidade da resposta em relação a quantidade de elementos de contorno usada na discretização das placas. Em geral, para se obter boa concordância com os resultados da literatura, foi necessário o uso de um grande número de elementos de contorno (em alguns casos, da ordem de milhares de elementos). Este fato pode ser explicado pelo uso de elementos de baixa ordem (elementos constantes). Espera-se uma convergência mais rápida se elementos de maior ordem (elementos quadráticos, por exemplo) forem usados. Entretanto, esta análise ficou fora do escopo deste trabalho.

Os resultados foram comparados com soluções analíticas e numéricas disponíveis na literatura. No geral, a formulação desenvolvida apresentou um bom desempenho na modelagem de problemas dinâmicos, constituindo-se, como uma alternativa para a análise de estruturas tridimensionais finas sob cargas transientes. 


\subsection{Sugestões para trabalhos futuros}

Neste trabalho apenas o cálculo de deslocamentos e forças de superfície foram realizados para problemas estáticos e dinâmicos. Desta maneira sugere-se como possibilidade de trabalhos futuros:

- Para a formulação dinâmica, análise da sensibilidade dos resultados quanto a quantidade e posição dos pontos internos e ao tamanho dos passos de tempo.

- Troca do elemento por um de maior ordem, como os elementos quadráticos, por exemplo.

- A inclusão do cálculo de tensões;

- Extensão da formulação para análise modal de estruturas constituídas pela associação de placas planas no espaço.

- Extensão da formulação para a análise flambagem linear de estruturas constituídas pela associação de placas planas no espaço.

- Extensão da formulação para análise de pós-flambagem de estruturas constituídas pela associação de placas planas no espaço.

- Extensão da formulação para análise de estruturas constituídas pela associação de placas planas no espaço considerando deformações finitas (grandes deslocamentos/deformações). 


\section{Referências Bibliográficas}

E. L. Albuquerque, P. Sollero, W. Venturini, and M. H. Aliabadi. Boundary element analysis of anisotropic kirchhoff plates. International Journal of Solids and Structures, 43:4029-4046, 2006.

P. M. Baiz and M. H. Aliabadi. Local buckling of thin-walled structures by the boundary element method. Engineering Analysis with Boundary Elements, 33:302-313, 2009a.

P.M. Baiz and M.H. Aliabadi. Local buckling of thin-walled structures by the boundary element method. Engineering Analysis with Boundary Elements, 33(3):302 - 313, 2009b. ISSN 0955-7997.

C. Brebbia and J. Dominguez. Boundary Element an Introductory Course. Computation Mechanics Publications, Southampton, Boston, second edition, 1989.

L. S. Campos. Análise de tensões em placas finas laminadas sob carregamento dinâmico usando o método dos elementos de contorno. PhD thesis, Universidade de Brasília, Brasília, 2012.

F. Chirino, R. Gallego, A. Saes, and J. Dominguez. A comparative study of three boundary element approaches to transient dynamic crack problems. IEngineering Analysis with Boundary Elements, 13:11-19, 1994.

D. I. G. Costa. Nova Formulação do Método dos Elementos de Contorno para Associação de Placas Espessas. PhD thesis, Universidade de Brasília, Campus Darcy Ribeiro, 2015. In Portuguese.

C. Di Pisa and M. H. Aliabadi. Fatigue crack growth analysis of assembled plate structures with dual boundary element method. Engineering Fracture Mechanics, pages 200-213, 2013.

C. Di Pisa and M. H. Aliabadi. Boundary element analysis of stiffened panels with repair patches. Engineering Analysis with Boundary Elements, 56:162-175, 2015. 
C. Di Pisa, M. H. Aliabadi, and A. Young. Boundary element method analysis of assembled plate structures undergoing large deflection. Journal of Strain Analysis for Engineering Design, 45:179-195, 2010.

C. Di Pisa, M. H. Aliabadi, and A. Young. Dual boundary method for assembled plate structures undergoing large deflection. International Journal for Numerical Methods in Engineering, 89:1720-1738, 2012.

T. Dirgantara and M. H. Aliabadi. Boundary element analysis of assembled plate structures. Communications in Numerical Methods in Engineering, 17(10):749-760, 2001. ISSN 1099-0887. doi: 10.1002/cnm.446.

J. Dominguez. Boundary Elements in Dynamics. Computational Mechanics Publications, Southampton, 1993.

P. Fedelinsk, M. H. Aliabadi, and D. P. Rooke. Boundary element formulations for the dynamic analysis of cracked structures. Engineering Analysis with Boundary Elements, 17:45-56, 1996.

G. B. Fernandes. Introdução a Teoria das Placas Elásticas. Universidade Estadual de Campinas, Limeira-SP, 1974.

X. Gao. The radial integration method for evaluation of domain integrals with boundary only discretization. Engn. Analysis with Boundary Elements, 26:905-916, 2002.

M. A. Golberg, C. S. Chen, and H. Bowman. Some recent results and proposals for the use of radial basis functions in the bem. Engineering Analysis with Boundary Element, 23:285-296, 1999.

S. S. Hoefel. Análise dinâmica de estruturas 3d formadas por laminados compósitos através do método dos elementos de contorno. Technical report, Universidade Estadual de Campinasy, Relatório novembro, 2006.

S. S. Hoefel, E. L. Albuquerque, and P. Sollero. Modeling thin walled 3D composite structures using the boundary element method. In A. P. Selvadurai, C. L. Tan, and M. H. Aliabadi, editors, Advances in Boundary Element and Meshless Techniques VI, 2005.

J. C. Houbolt. A reccurrence matrix solution for the dynamic response of elastic aircraft. J. of Aeronautical and Science, 17:540-550, 1950.

James H. Kane. Boundary Element Analysis in Engineering Continuum Mechanics. Prentice Hall, New Jersey, 1993. 
G. R. Kirchhoff. On the equilibrium and motion of an elastic plate. J. Math., 40:51-58, 1850. In German.

C. Loeffler and W. J. Mansur. Analysis of time integration schemes for boundary element applications to transient wave propagation problems. In C. A. Brebbia and W. S. Venturini, editors, Boundary Element techniques: Applications in stress analysis and heat transfer, pages 105-122, Computational Mechanics Publications, Southhampton, 1987.

R. D. Mindlin. Influence of rotatory inertia and shear on flexural motions of isotropic elastic plates. Journal of Applied Mechanics., 18:31-38, 1951.

J. B. Paiva. Boundary element formulation for plate bending and its aplication in engineering. PhD thesis, University of São Paulo, São Carlos School of Engineering, 1987. In Portuguese.

W. P. Paiva, P. Sollero, and E. L. Albuquerque. Treatment of hypersingularities in boundary element anisotropic plate bending problems. Latin American Journal of Solids and Structures, 1:49-73, 2003.

C. Di Pisa. Boundary Element Analysis of Multi-layered Panels and Structures. Phd, 2005.

S. D. Poisson. Tmemoire sur l'equilibre et le mouvement des corps solides. Journal of Mathematics Physics, 12:8, 1829.

C. Rajamohan and J. Raamachandran. Bending of anisotropic plates by charge simulation method. Advances in Engn. Software, 30:369-373, 1999.

Y.F. Rashed. Boundary element formulations for thick plates. Topics in engineering. WIT Press, 2000. ISBN 9781853126284.

E. Reissner. On the theory of bending elastic plates. J. of Mathematical Physics, 23: $184,1944$.

M.H. Sadd. Elasticity: Theory, Applications, and Numerics. Elsevier Science, 2009. ISBN 9780080922416.

L. C. F. Sanches, E. Mesquita, R. Pavanello, and L. Palermo. Dynamic stationary response of reinforced plates by the boundary element method. Mathematical Problems in Engineering, 17(62157), 2007. doi: 10.1155/2007/62157.

G. Shi and G. Bezine. Buckling analysis of orthotropic plates by boundary element method. Mechanics Research Communications, 26:1351-1370, 1990. 
J. Sladek, V. Sladek, C. Zhang, J. Krivacek, and P. H. Wen.

M. Tanaka, T. Matsumoto, and A. Shiozaki. Application of boundary-domain element method to the free vibration problem of plate structures. Computers and Structures, 66(6):725 - 735, 1998. ISSN 0045-7949. doi: http://dx.doi.org/10.1016/ S0045-7949(97)00129-6.

S. Timoshenko and S. Woinowski-Krieger. Theory of Plates and shells. McGraw-Hill, New York, 1959.

W. S. Venturini. A study of boundary element method and its application on engineering problems. PhD thesis, University of São Paulo, São Carlos School of Engineering, 1988. In Portuguese.

J. Wang and K. Schweizerhof. The fundamental solution of moderately thick laminated anisotropic shallow shells. Int. J. Engng. Sci., 33:995-1004, 1995.

J. Wang and K. Schweizerhof. Free vibration of laminated anisotropic shallow shells including transverse shear deformation by the boundary-domain element method. Computers and Structures, 62:151-156, 1997.

F. Vander Weën. Application of the boundary integral equation method to reissner's plate model. International Journal for Numerical Methods in Engineering, 18(1): 1-10, 1982. ISSN 1097-0207. doi: 10.1002/nme.1620180102. URL http://dx.doi. org/10.1002/nme.1620180102.

B. C. Wu and N. J. Altiero. A new numerical method for the analysis of anisotropic thin plate bending problems. Computer Meth. in Appl. Mechanics and Engineering, 25:343-353, 1981. 